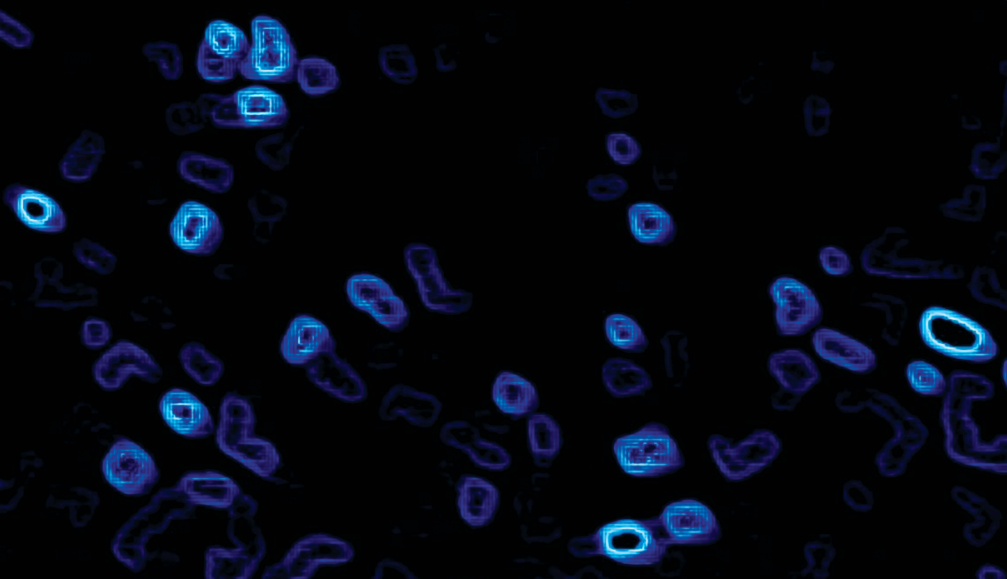

Q0e

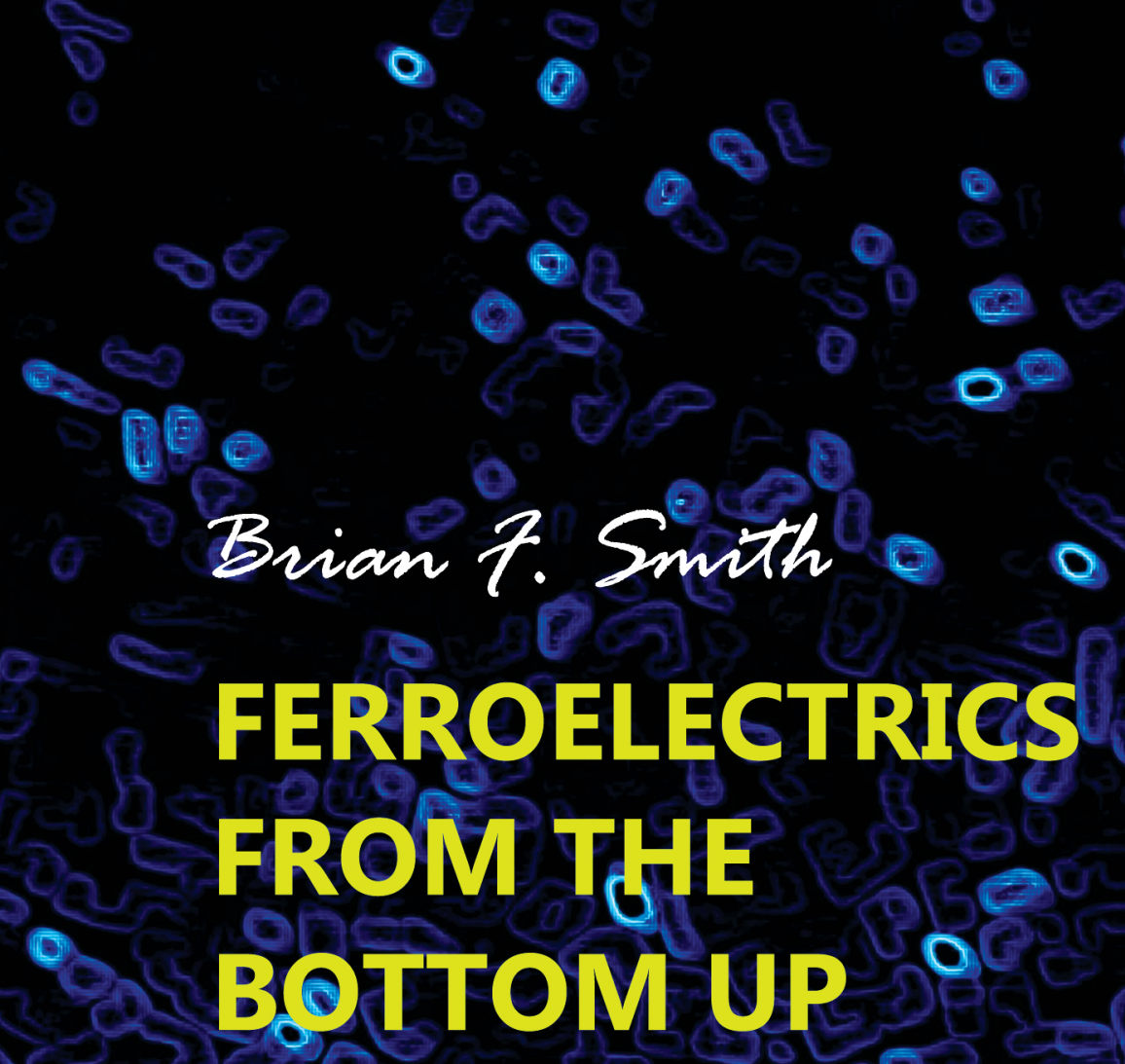

Investigation of Nanoscale Boundary Conditions in Ferroelectric Thin Films Using Novel Bottom-up Growth Techniques

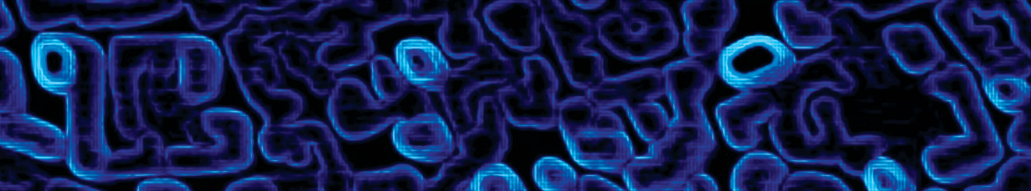




\title{
Ferroelectrics From The Bottom Up
}

\author{
Investigation of Nanoscale Boundary \\ Conditions In Ferroelectric Thin Films Using \\ Novel Bottom-up Growth Techniques
}

Brian F. Smith 


\section{Ph.D. Committee}

Chairman and Secretary:

Prof. dr.ir. J.W.M. Hilgenkamp

Supervisor:

Prof. dr. ing. A.J.H.M. Rijnders

Assistant-supervisors:

Prof. dr. ir. J.E. ten Elshof

Dr. ir. G. Koster

Members:

Prof. dr. ing. D.H.A Blank

Prof .dr. ing. W.G. van der Wiel

Prof. dr. B. Noheda

Prof. dr. J. Verbeeck

Cover:

Inclination edge detection of the surface topography of $\mathrm{PbTiO}_{3}$ on $\mathrm{Ca}_{2} \mathrm{Nb}_{3} \mathrm{O}_{10}$ nanosheets imaged by tapping mode atomic force microscopy.

The research described in this thesis was carried out within the Inorganic Materials Science group, Department of Science and Technology, and the MESA+ Institute for Nanotechnology at the University of Twente. This research was carried out under project number M62.2.08SDMP24 in the framework of the Research Program of the Materials innovation institute (M2i) and the Foundation for Fundamental Research on Matter (FOM) in the Netherlands

Ph.D. Thesis, University of Twente

Copyright (C) 2014 by B. F. Smith

DOI: $10.3990 . / 1.9789036537360$

ISBN: 978-90-365-3736-0 


\title{
FERROELECTRICS FROM THE BOTTOM UP
}

\section{INVESTIGATION OF NANOSCALE BOUNDARY CONDITIONS IN FERROELECTRIC THIN FILMS USING NOVEL BOTTOM-UP GROWTH TECHNIQUES}

\section{DISSERTATION}

\author{
to obtain \\ the degree of doctor at the University of Twente \\ on the authority of the rector magnificus \\ Prof. dr. H. Brinksma \\ on account of the decision of the graduation committee, \\ to be publicly defended \\ on Thursday 04 September 2014 at 16:45
}

by:

Brian Filemyr Smith

born on the $16^{\text {th }}$ of October 1984

in Silver Spring, Maryland 
This dissertation has been approved by promoter:

Prof. dr. ing. A.J.H.M. Rijnders

and assistant promoters:

Prof. dr. ir. J.E. ten Elshof

Dr. ir. G. Koster 


\section{Contents}

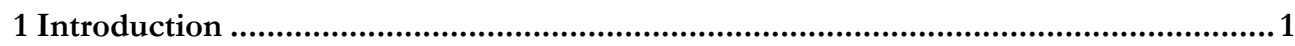

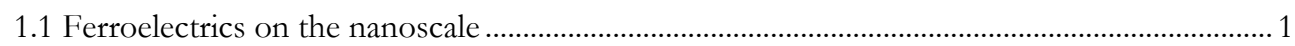

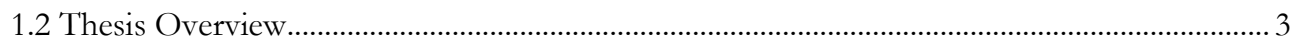

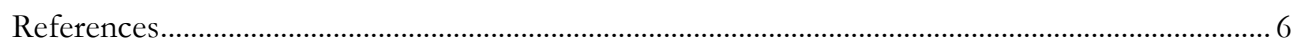

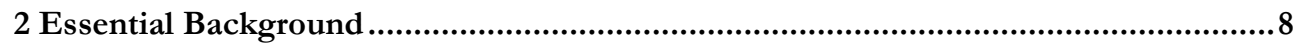

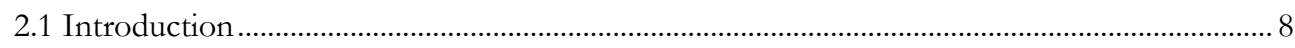

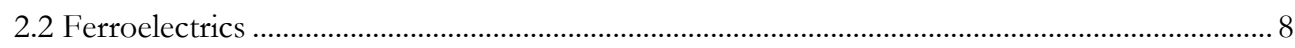

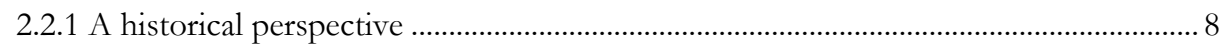

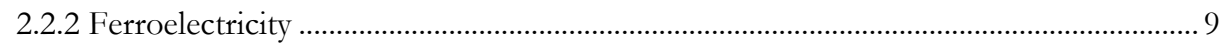

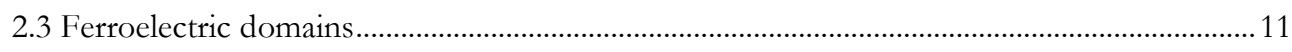

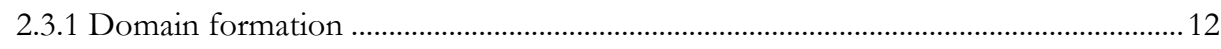

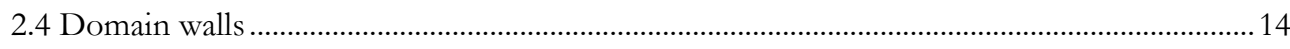

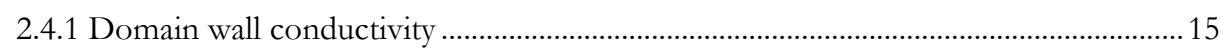

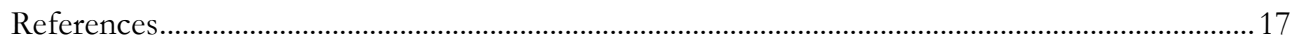

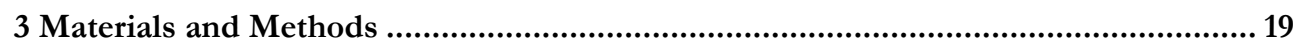

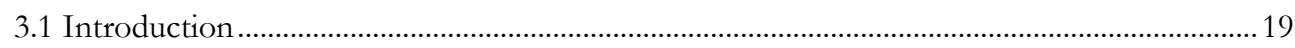

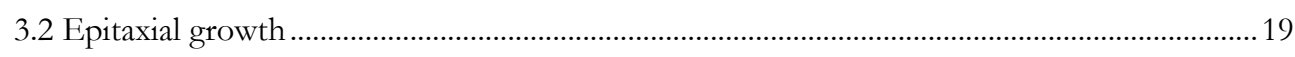

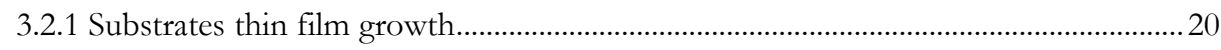

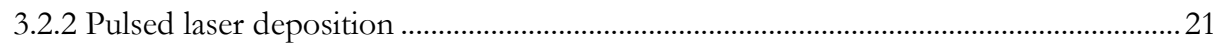

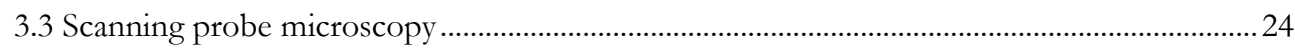

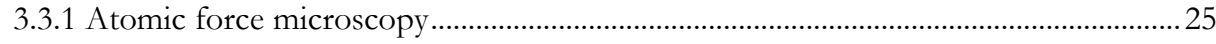

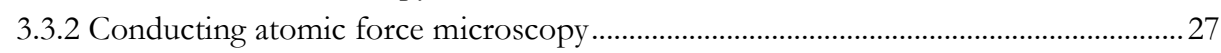

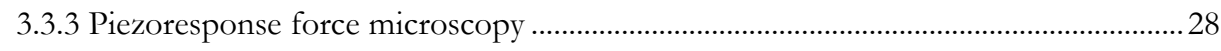

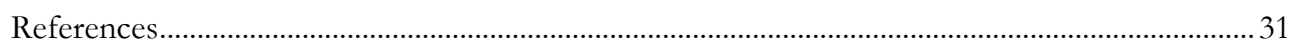

4 Piezoresponse Force Microscopy in Ultra High Vacuum ..................................... 33

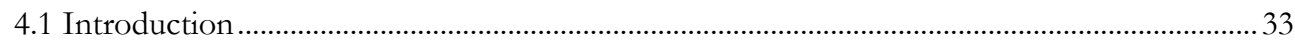

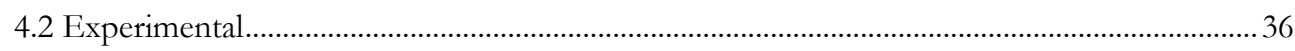

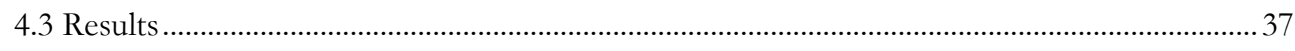

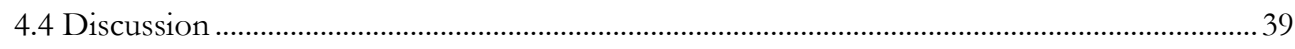

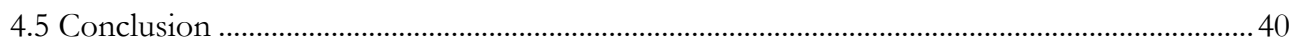

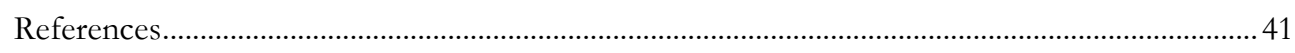




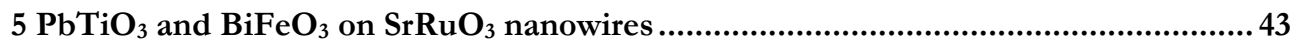

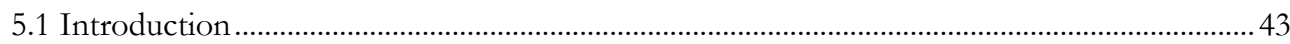

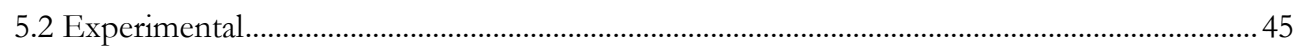

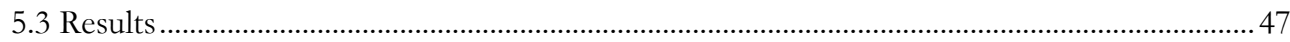

5.3.1 Growth of $\mathrm{PbTiO}_{3}$ and $\mathrm{BiFeO}_{3}$ on $\mathrm{SrRuO}_{3}$ nanowires ................................................ 47

5.3.2 Domain structure of $\mathrm{PbTiO}_{3}$ and $\mathrm{BiFeO}_{3}$ on $\mathrm{SrRuO}_{3}$ nanowires ............................... 49

5.3.3 Growth morphology of $\mathrm{PbTiO}_{3}$ and $\mathrm{BiFeO}_{3}$ on $\mathrm{SrRuO}_{3}$ islands ................................. 53

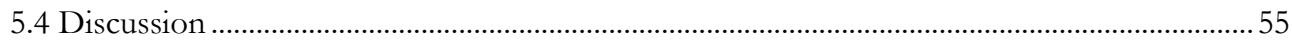

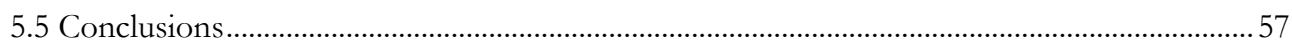

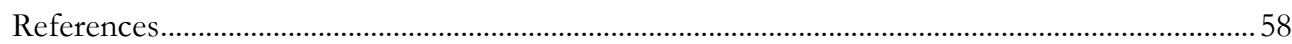

$6 \mathrm{BiFeO}_{3}$ Domain Wall Conductivity using $\mathrm{SrRuO}_{3}$ Nanowires ................................. 61

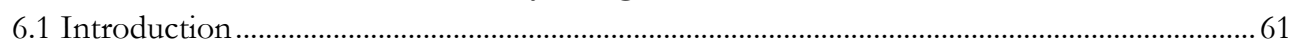

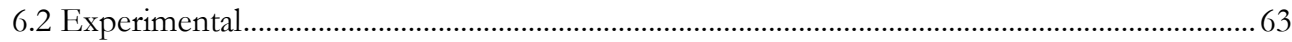

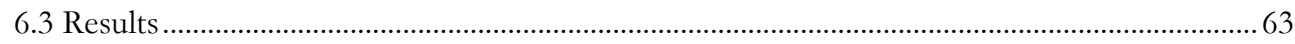

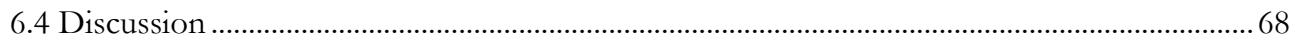

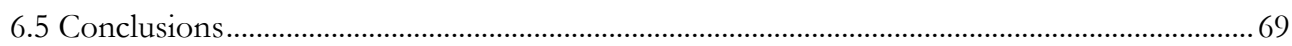

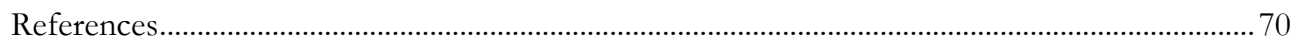

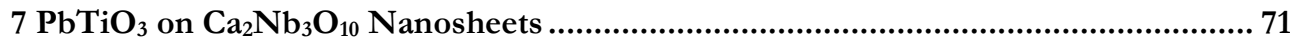

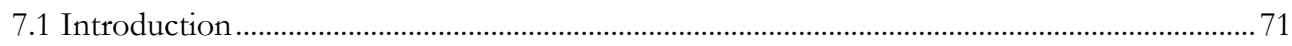

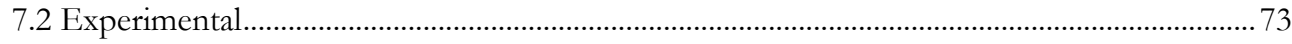

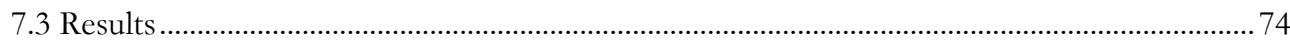

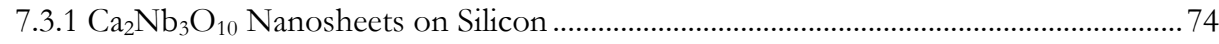

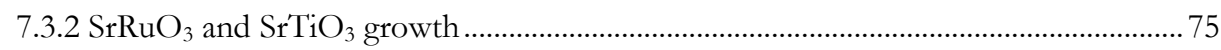

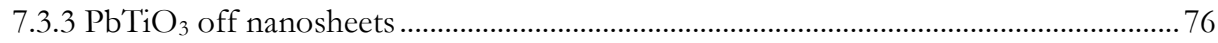

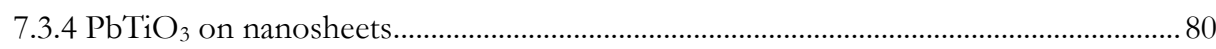

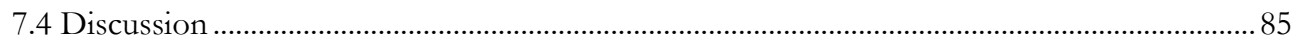

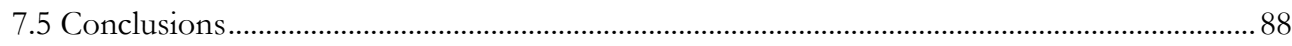

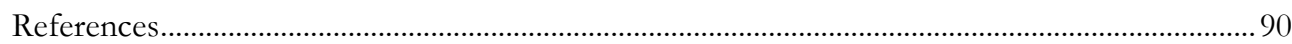

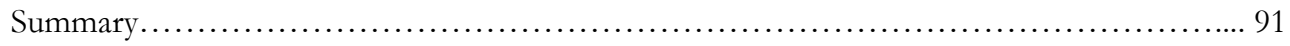

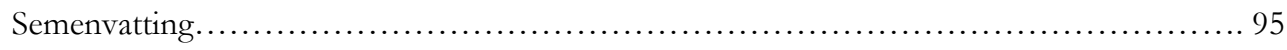

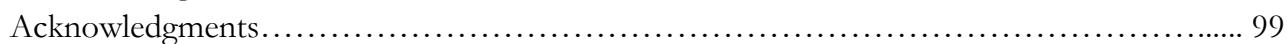





\section{Chapter 1}

\section{Introduction}

\subsection{Ferroelectrics on the nanoscale}

In modern society, ferroelectric materials are ubiquitous in many technological domains. One of the first and most used application for ferroelectrics is as a high dielectric material for capacitors [1]. However, what has made ferroelectrics such an intensely researched class of materials is the wide range of other properties that ferroelectrics exhibit. By taking advantage of these properties additional functionality can be added to devices, extending their range of applications. For example, ultrasensitive infrared detectors utilize the pyroelectricity of ferroelectrics[2]. The high piezoelectric coefficients exhibited by many ferroelectrics make these materials ideal for use as actuators and sensors for Micro-ElectroMechanical Systems (MEMS) [3]. Finally, the reversible spontaneous polarization in ferroelectrics can be used to store information allowing the development of Ferroelectric Random Access Memories (FeRAM) which are non-volatile, have the advantage of lower power consumption and faster read/write times compared to other random access memory schemes [4].

The application of ferroelectrics into many electronic devices and their subsequent integration with semiconductor processing technologies produces a drive to make the ferroelectric elements smaller and smaller. The recent demand for portable consumer electronic devices with a wide range of functionalities, e.g. tablets and smart phones, increases the drive to make the ferroelectric components smaller and is now reaching the dimensions on the nanoscale, $10^{-9}$ meters. Therefore, it is vital to understand how ferroelectric behave at these dimensions where quantum effects and the increased surface to volume ratio are known to drastically change the properties of many materials. As a result, the study of nanoscale ferroelectrics focuses on how the crystal structure and functional properties of ferroelectrics change as these materials are patterned into objects having one or more dimensions confined to the nanoscale from thin films (2D) to nanowires (1D) to nanodots (0D).

For many years the idea of nanoscale ferroelectrics was considered impossible. It was thought that there was a minimum size requirement in order for ferroelectricity to exist [5-7]. 
Over the years this critical size became smaller and smaller as improvements in fabrication techniques improved control of the growth of nanoscale ferroelectrics. Initially research into nanoscale ferroelectricity was focused mainly on 2D thin and ultra-thin films. Precise control of the ferroelectric growth conditions through advances in thin film growth techniques such as molecular beam epitaxy and pulsed laser deposition has enabled the growth ferroelectric films down to of only a few unit cells [8-11].

Lateral size effects in ferroelectrics have been much less investigated topic due to difficulties fabricating and characterizing nanoscale objects of complex oxides. Advances in fabrication of ferroelectric nanostructures through various techniques and development of characterization tools allowing the polarization to be explored with nanoscale resolution has enabled researchers to be able to investigate nanoscale ferroelectrics beyond thin films. There have been two general approaches to the fabrication of ferroelectric nanostructures; top-down and bottom-up. In top-down techniques a larger quantity of material is grown followed by removal of material until nanosized ferroelectric objects are created. Standard photolithography is an example of a top-down method. However, this specific method cannot be used to create nanosized structures due to the wavelength of visible light being 700-390 nm. However, sub-100 nm features can be patterned by using ultraviolet or extreme ultraviolet light, although the optics required for UV and EUV light greatly increase the cost of these systems. Besides photolithography, electron or ion beams are used in techniques such as Focused Ion Beam (FIB) milling and Electron Beam (EB) writing. First successfully demonstrated for the creation of ferroelectric nanostructures in 1999 [12] FIB has been a particularly popular research method to create nanoscale capacitors. The drawback of topdown techniques is the inherent destructive nature of the process. The surface layers of the ferroelectric nanostructure can be damaged by the beam used to remove material and can be contaminated by resputtering. Furthermore, the serial nature of these techniques means that large scale application is impractical. Bottom-up approaches do not suffer from the drawbacks of the top-down techniques as they utilize naturally occurring self-assembly processes to create nanosized objects. However, control over the size and shape and position of the resulting nanoscale features is an issue.

Researchers have used the techniques discussed above to begin to investigate the behavior of ferroelectric materials as their lateral dimensions enter the nanoscale. Using top down approaches several studies observed an increase in the piezoresponse of ferroelectric nanoislands with dimensions below $300 \mathrm{~nm}$. This increase was attributed to a reduced substrate clamping at small dimensions [13] and improved electrical properties due to a lack of a domains [14]. These results were also obtained using bottom-up techniques [15]. Bottom-up approaches have also been utilized to investigate the critical volume for ferroelectricity. Using chemical solution deposition [16] and metalorganic chemical vapor deposition [17] ferroelectric particles with sizes down to $1.7 \mathrm{~nm}$ thick and $38 \mathrm{~nm}$ wide were shown to remain ferroelectric. 
When a ferroelectric is patterned into a nanoscale object the material response is affected by surface effects and a change in the crystal structure of the ferroelectric. The act of patterning the ferroelectric changes the boundary conditions, the electrical and mechanical forces acting on the ferroelectric, resulting in size effects. Therefore, an alternative method of studying nanoscale ferroelectrics, and the method employed in this thesis, is to investigate the response of a ferroelectric film to nanoscale changes in the boundary conditions under which the ferroelectric is subjected. The most direct way to accomplish this approach is to engineer on the nanoscale either the top or bottom interface of the ferroelectric film. In terms of the ferroelectric boundary conditions this means changing the conductivity and or strain of the interface with nanoscale dimensions either by modifying the existing interface or selectively adding material to the interface. As a result, nanoscale variations in the electrical and or mechanical boundary conditions of the ferroelectric film are achieved without any removal of material. This approach has the benefit of eliminating post processing of the ferroelectric material which removes extrinsic effects from the investigation of the ferroelectric properties. In addition, by using self-assembly techniques to engineer the bottom interface the entire sample is grown in a single deposition run creating high quality structures which can be analyzed in situ further reducing extrinsic effects due to contamination from the atmosphere which can have a profound effect on the properties of nanoscale structures.

The reason this approach has not been realized until now is the challenge of creating an engineered surface with nanoscale dimension while maintaining a high quality epitaxial ferroelectric layer. However, recently two new techniques have been developed that enable the patterning of a bottom interface that will allow subsequent deposition of a high quality ferroelectric thin film. The first of these techniques uses an ordered difference in the chemical surface of a oxide substrate for selective growth of $\mathrm{SrRuO} 3$ into nanowires [18]. The second novel method employs a layer of oxide nanosheets of a single unit cell thickness to locally grow single crystalline oxide films [19]. Using these two novel self-assembly methods ferroelectric thin films can now be grown on engineered bottom electrodes where the local boundary conditions which the ferroelectric film is subjected changes on the nanoscale.

\subsection{Thesis overview}

This thesis will explore nanoscale size effects in the ferroelectric materials $\mathrm{PbTiO}_{3}$ and $\mathrm{BiFeO}_{3}$ using several different approaches. To start, Chapter 2 will provide a background into the core concepts related to the study of nanoscale ferroelectrics going into detail about what makes a material ferroelectric, what are ferroelectric domains and what causes their formation, and the phenomena of conduction at domain walls in ferroelectrics. Chapter 3 deals with the materials and methods used to carry out the experiments in the later chapters providing information on how the materials in this thesis are grown and characterized. Chapter 4 will examine the effect on the spatial resolution of in situ characterization of 
ferroelectric domains using Piezoresponse Force Microscopy. The use of in situ characterization allows the intrinsic properties of the nanoscale ferroelectrics to be accurately studies as there is no contamination due to exposure of the material to atmosphere. Determination of the spatial resolution of the technique provides a concrete limit to the length scale that is accessible in this study.

Chapters 5, 6, and 7 are devoted to the study of nanoscale ferroelectrics using the two new bottom-up growth techniques; growth of $\mathrm{PbTiO}_{3}$ and $\mathrm{BiFeO}_{3}$ thin films on conducting $\mathrm{SrRuO}_{3}$ nanowires and the growth of $\mathrm{PbTiO}_{3}$ on $\mathrm{Ca}_{2} \mathrm{Nb}_{3} \mathrm{O}_{10}$ nanosheets. These two approaches, growth on nanowires and nanosheets, have the major advantage over traditional patterning techniques of being able to produce nanoscale ferroelectric objects without the need for any post processing after the ferroelectric growth. This eliminates extrinsic processing effects allowing the direct investigation of the intrinsic ferroelectric properties of these samples. In Chapter $5 \mathrm{PbTiO}_{3}$ and $\mathrm{BiFeO}_{3}$ thin films are grown conducting $\mathrm{SrRuO}_{3}$ nanowires. The choice to use conducting nanowires on an insulating substrate as a bottom electrode is done to periodically modulate the boundary conditions of the ferroelectric film along the lateral direction perpendicular to the nanowires. As a result, regions of the ferroelectric on and off of the $\mathrm{SrRuO}_{3}$ nanowires will experience difference depolarizing fields depending on whether the bound surface charges are screened by a nanowire or not. Furthermore, the high aspect ratio of the nanowires will locally strain the film around the edges of the nanowires as they are conformally coated by the ferroelectric film Piezoresponse force microscopy and transmission electron microscopy are used to investigate how the ferroelectric domains are oriented in response to these novel boundary conditions. Finally, by comparing $\mathrm{PbTiO}_{3}$ and $\mathrm{BiFeO}_{3}$ films this chapter will demonstrate how the growth kinetics of the ferroelectric film on $\mathrm{SrRuO}_{3}$ nanowires has a profound influence on the resulting domain structure. In Chapter 6 the $\mathrm{SrRuO}_{3}$ nanowires are used as local electrical contacts to study domain wall conductivity, particularity how the conductivity of a domain wall in $\mathrm{BiFeO}_{3}$ changes with domain wall length providing new information on the origin of the domain wall conduction mechanism. In Chapter 7 the influence of changing lateral dimensions on the properties of nanoscale ferroelectrics is investigated by growing $\mathrm{PbTiO}_{3}$ on single unit cell thick $\mathrm{Ca}_{2} \mathrm{Nb}_{3} \mathrm{O}_{10}$ nanosheets on a silicon substrate. The $\mathrm{Ca}_{2} \mathrm{Nb}_{3} \mathrm{O}_{10}$ nanosheets have lateral sizes from $2 \mu \mathrm{m}$ down to $100 \mathrm{~nm}$ and are used as a seed layer to grow epitaxial nanoscale ferroelectric objects which will have the same size and dimensions as the initial nanosheets. $\mathrm{PbTiO}_{3}$ not deposited on the nanosheets results in polycrystalline growth. The difference between the polycrystalline and epitaxial growth is easily differentiated by the surface morphology imaged using atomic force microscopy. This direct patterning using $\mathrm{Ca}_{2} \mathrm{Nb}_{3} \mathrm{O}_{10}$ nanosheets as a seed layer eliminates post deposition patterning and processing enabling in situ characterization eliminating extrinsic contributions allowing the intrinsic ferroelectric properties to be studied. Finally, the different size, dimension, and packing density of the initial nanosheet layer allows, in a single sample, the study of a wide range of influences on the domain structure and piezoelectric response of the nanoscale $\mathrm{PbTiO}_{3}$ covered nanosheets. The 
domain structure and piezoresponse of the nanoscale epitaxial $\mathrm{PbTiO}_{3}$ covered nanosheets is investigated using piezoresponse force microscopy and shows a markedly different behavior from the ferroelectric size effect observed using traditional top down patterning techniques. 


\section{References}

[1] J. Scott, "Applications of modern ferroelectrics," Science, vol. 315, pp. 954-959, 2007.

[2] B. Willing, M. Kohli, P. Muralt, N. Setter, and O. Oehler, "Gas spectrometry based on pyroelectric thin-film arrays integrated on silicon," Sensors and Actuators A: Physical, vol. 66, pp. 109-113, 1998.

[3] P. Muralt, "Ferroelectric thin films for micro-sensors and actuators: a review," Journal of Micromechanics and Microengineering, vol. 10, p. 136, 2000.

[4] J. F. Scott and C. A. P. De Araujo, "Ferroelectric memories," Science, vol. 246, pp. 1400-1405, 1989.

[5] K. Ishikawa, K. Yoshikawa, and N. Okada, "Size effect on the ferroelectric phase transition in PbTiO 3 ultrafine particles," Physical Review B, vol. 37, p. 5852, 1988.

[6] S. Tsunekawa, S. Ito, T. Mori, K. Ishikawa, Z. Q. Li, and Y. Kawazoe, "Critical size and anomalous lattice expansion in nanocrystalline BaTiO_\{3\} particles," Physical Review B, vol. 62, pp. 3065-3070, 08/01/ 2000.

[7] K. Uchino, E. Sadanaga, and T. Hirose, "Dependence of the crystal structure on particle size in barium titanate," Journal of the American Ceramic Society, vol. 72, pp. 15551558, 1989.

[8] D. D. Fong, C. Cionca, Y. Yacoby, G. B. Stephenson, J. A. Eastman, P. H. Fuoss, et al., "Direct structural determination in ultrathin ferroelectric films by analysis of synchrotron x-ray scattering measurements," Physical Review B, vol. 71, p. 144112, 04/27/ 2005.

[9] D. D. Fong, G. B. Stephenson, S. K. Streiffer, J. A. Eastman, O. Auciello, P. H. Fuoss, et al., "Ferroelectricity in ultrathin perovskite films," Science, vol. 304, pp. 1650 1653, Jun 112004.

[10] L. Despont, C. Lichtensteiger, F. Clerc, M. G. Garnier, F. J. G. de Abajo, M. A. Van Hove, et al., "X-ray photoelectron diffraction study of ultrathin PbTiO3 films," European Physical Journal B, vol. 49, pp. 141-146, Jan 2006.

[11] P. Maksymovych, M. Huijben, M. Pan, S. Jesse, N. Balke, Y.-H. Chu, et al., "Ultrathin limit and dead-layer effects in local polarization switching of BiFeO3," Physical Review B, vol. 85, Jan 312012.

[12] C. S. Ganpule, A. Stanishevsky, Q. Su, S. Aggarwal, J. Melngailis, E. Williams, et al., "Scaling of ferroelectric properties in thin films," Applied Physics Letters, vol. 75, pp. 409-411, Jul 191999.

[13] V. Nagarajan, A. Roytburd, A. Stanishevsky, S. Prasertchoung, T. Zhao, L. Chen, et al., "Dynamics of ferroelastic domains in ferroelectric thin films," Nature Materials, vol. 2, pp. 43-47, Jan 2003.

[14] S. Buhlmann, B. Dwir, J. Baborowski, and P. Muralt, "Size effect in mesoscopic epitaxial ferroelectric structures: Increase of piezoelectric response with decreasing feature size," Applied Physics Letters, vol. 80, pp. 3195-3197, Apr 292002. 
[15] H. Han, K. Lee, W. Lee, M. Alexe, D. Hesse, and S. Baik, "Fabrication of epitaxial nanostructured ferroelectrics and investigation of their domain structures," Journal of Materials Science, vol. 44, pp. 5167-5181, Oct 2009.

[16] A. Roelofs, T. Schneller, K. Szot, and R. Waser, "Towards the limit of ferroelectric nanosized grains," Nanotechnology, vol. 14, pp. 250-253, Feb 2003.

[17] H. Fujisawa, K. Morimoto, M. Shimizu, and H. Niu, "Observations of island structures at the initial growth stage of PbZrxTi1-xO3 thin films prepared by metalorganic chemical vapor deposition," Japanese Journal of Applied Physics Part 1Regular Papers Short Notes \& Review Papers, vol. 39, pp. 5446-5450, Sep 2000.

[18] B. Kuiper, J. L. Blok, H. J. Zandvliet, D. H. Blank, G. Rijnders, and G. Koster, "Selforganization of $\mathrm{SrRuO} 3$ nanowires on ordered oxide surface terminations," MRS Communications, vol. 1, pp. 17-21, 2011.

[19] M. Nijland, S. Kumar, R. Lubbers, D. H. Blank, G. Rijnders, G. Koster, et al., "Local Control over Nucleation of Epitaxial Thin Films by Seed Layers of Inorganic Nanosheets," ACS applied materials \& interfaces, 2014. 


\section{Chapter 2}

\section{Essential Background}

\subsection{Introduction}

This chapter serves as a general introduction to the field of ferroelectric materials to establish important concepts that are used and expanded on throughout the remainder of this thesis. First a brief historical perspective of the discovery and use of ferroelectrics is presented to understand the important role ferroelectrics have and do play in society. Then a definition and description of what is ferroelectricity starting with a description of what is a crystal and how specific distortions of a crystal results in ferroelectricity. From here the concept of ferroelectric domains is introduced followed by a description of how and why these domains form. Next we will introduce nanoscale ferroelectricity to examine what happens to ferroelectric materials when dimensions of the materials are limited to nanometer sizes. Finally this chapter will conclude with a section examining interesting phenomena that occurs at the domain walls, particularly domain wall conductivity. With the background information presented in this chapter the reader has a solid footing upon which to understand the work and conclusions throughout this thesis.

\subsection{Ferroelectrics}

\subsubsection{A historical perspective}

The discovery of ferroelectricity occurred in 1922 in Rochelle salt $\left(\mathrm{KNaC}_{4} \mathrm{H}_{4} \mathrm{O}_{6} \cdot 4 \mathrm{H}_{2} \mathrm{O}\right)$ by Valasek [1]. Rochelle salt was one of the first piezoelectric materials to be discovered and was a heavily studied material at the time due to its use as transducer for gramophones and microphones. The name given to the newly discovered phenomena ferroelectricity can be misleading as the prefix "ferro" comes from the Latin word for iron of which Rochelle salt contains none. Instead the name was chosen to reflect the analogous behavior between ferroelectricity and ferromagnetism (where iron is a ferromagnetic material). It is interesting to note that the discovery of ferroelectric materials occurred so late considering ferromagnetic materials had been used for over two thousand years in compasses. Until the 
discovery of ferroelectricity in $\mathrm{BaTiO}_{3}[2,3]$ in the 1940s ferroelectricity was considered an academic curiosity that could only be achieved with hydrogen bonding. Research into $\mathrm{BaTiO}_{3}$ took place and was accelerated by World War II as the US, Russia, and Japan looked for alternative high dielectric constant materials to mica for capacitors. From this point the electroceramic industry was born and is still billions of $\mathrm{BaTiO}_{3}$ capacitors are still being produced annually [4]. As the years have gone by ferroelectricity has been found in many different types of materials including polymers [5] and biological tissue [6].

The course of ferroelectrics changed again in the 1970s with the development of integrated circuits and research into ferroelectric thin films for memory devices began. Processing difficulties frustrated early research attempts in this direction. Advances in processing complex oxide thin films driven by research in high $T_{c}$ superconducting oxides provided fabrication methods for ferroelectric oxide thin film growth allowing ferroelectric memory devices to finally be realized. In the 1990s ferroelectrics became an important component in microelectromechanical systems (MEMS). The functionality of MEMS devices could be greatly expanded by the introduction of ferroelectric components for sensing and actuating. Today ferroelectrics are an important material in a wide range of devices from capacitors to nonvolatile memories to MEMS devices and much more [4, 7-10].

\subsubsection{Ferroelectricity}

A ferroelectric is a material which has a spontaneous switchable polarization below a critical temperature $T_{c}$. Polarization is merely the separation of positive and negative charge creating a dipole moment where the polarization direction points from negative to positive charge. Ferroelectricity gives rise to a characteristic polarization vs. electric field hysteresis curve shown in Figure 2.1. 


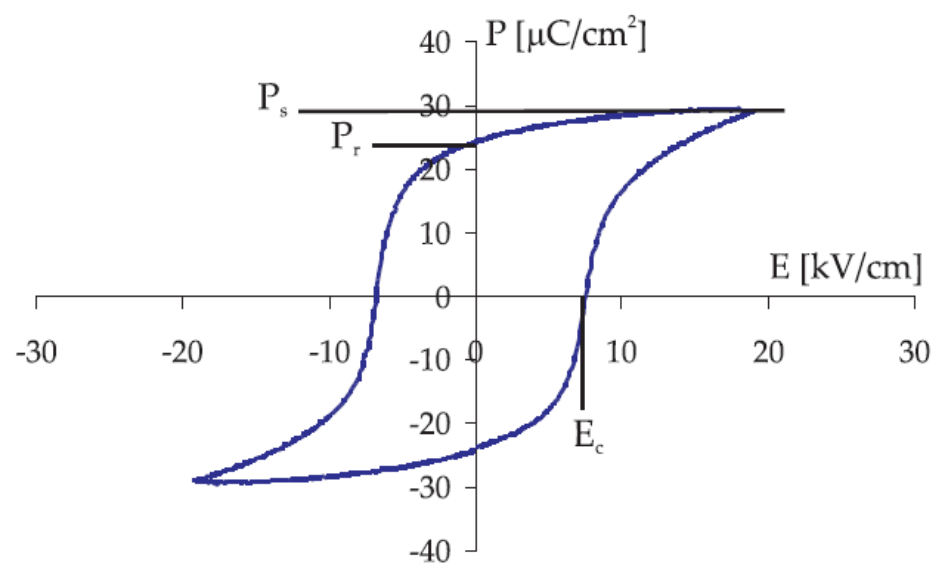

Figure 2.1 Ferroelectric bysteresis loop showing the saturation polarization $(\mathrm{Ps})$, remnant polarization $(\mathrm{Pr})$ and coercive field $(\mathrm{Ec})$

At zero applied field the ferroelectric has a nonzero polarization. By applying an electric field opposed to the polarization it is possible to switch the orientation of the polarization. All ferroelectrics are also members of two larger classes of materials pyroelectrics and piezoelectrics where polarization is created in response to a change in temperature and applied stress respectively.

Ferroelectricity is a property of the crystalline structure of the material and as such an understanding of ferroelectrics must begin with what is a crystal. For ferroelectrics the reverent definition of a crystal is a material whose constituent atoms, or molecules, are ordered periodically in three dimensions. In total there are 32 crystal classes that define all the atoms in a crystal can be arranged in space. Many properties including ferroelectric arise from the symmetry of the crystal structure. In order for a material to be a piezoelectric the crystal structure must lack an inversion center. Out of the 32 crystal classes 21 meet this requirement and are known as non-centrosymmetric crystals. In one other crystal class piezoelectricity is prohibited due to other symmetry considerations leaving 20 piezoelectric crystal classes. For a material to be pyroelectric further restrictions are place on the symmetry of the crystal. For pyroelectricity a crystal must have a polar axis which leaves 10 of the 20 crystal classes. There are no further symmetry considerations distinguishing ferroelectric from pyroelectric materials; if the polarization is switchable with the application of an electric field then the material is also ferroelectric.

How the displacements in a crystal results in switchable polarization is demonstrated using a typical perovskite crystal $\mathrm{ABO}_{3}$ shown in Figure 2.2. 


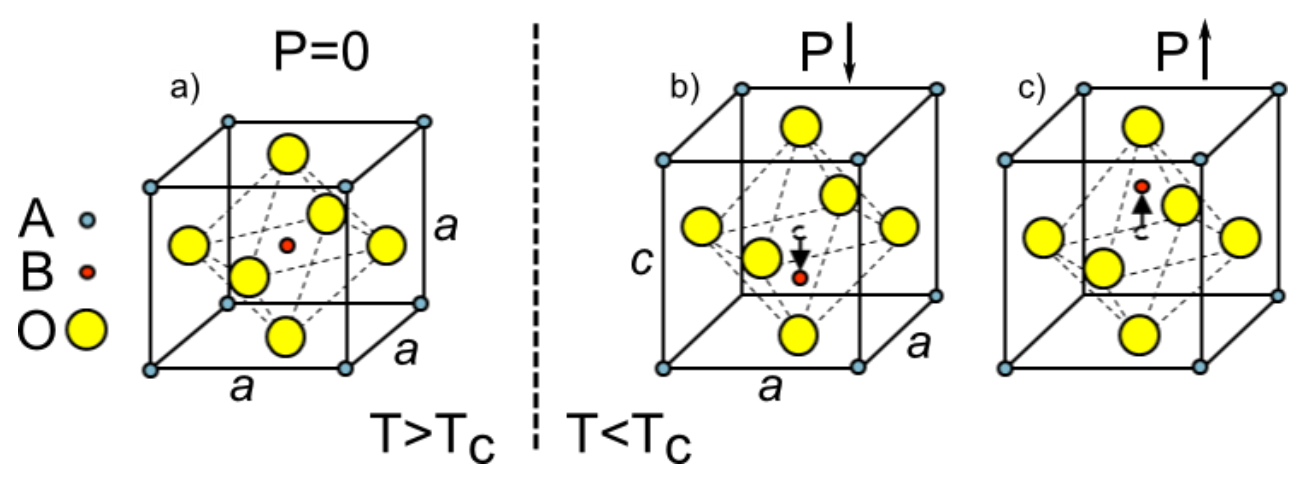

Figure 2.2 $\mathrm{ABO}_{3}$ perovskite crystal a) in the high temperature cubic phase b), c) low temperature phase with a tetragonal distortion in two different polarization states.

In this structure the A cations are located at the corners of the unit cell, the $\mathrm{b}$ cation is at the center, and the oxygen anions are positioned at the face centers. Above the transition temperature Tc the crystal has a cubic structure with all sides having length $a$. The charges of all the ions in the unit cell cancel out producing no separation of charge across the unit cell. Below Tc the unit cell becomes distorted and the charges in the unit cell no longer cancel each other resulting in a polarization developing across the unit cell. In Figure 2.2 a tetragonal distortion is shown where the unit cell is elongated in one direction now having length $c$. In this structure the central B atom no longer sits at the center of the unit cell but is displaced slightly along the long axis of the unit cell creating the polarization along this axis. The position the B ion is changed from "up" to "down" with the application of an electric field changing the direction of the polarization.

\subsection{Ferroelectric domains}

A ferroelectric domain is a region of a ferroelectric material where the polarization is oriented in the same direction and the boundary between adjacent domains are called domain walls. In an ideal infinite ferroelectric a single domain is the energetically favorable state for the material as the interface between two domains costs energy. Therefore domains arise from the presence of defects, strain, and the finite size of real materials. In discussing domains there are a few conventions that are used to effectively communicate information about the domains. First, ferroelectric domains are classified into two categories; pure ferroelectric domain and ferroelectric/ferroelastic domains. In a pure ferroelectric domain only the orientation of the polarization changes across domains. Ferroelectric/ferroelastic domains the change in the orientation of the polarization is accompanied by a change in the crystal lattice. To distinguish different domain structures domain walls are referred to by the change in angle of the polarization, for example $180^{\circ}$ or $90^{\circ}$ domains. The remainder of this section will deal with the factors that determine the formation of domains in a ferroelectric. 


\subsubsection{Domain formation}

Domain formation in a ferroelectric, as with many phenomena in material science, is all about minimizing free energy. Creating a domain wall in a ferroelectric costs energy. Therefore domains will only form when the creation a new domain lowers the free energy of the material more than the cost to create the domain wall. Consider a rectangular ferroelectric slab, the energy associated with a domain is proportional to the size of the domain; $E=U w$, where $U$ energy density of the domain and $w$ is the domain width. For domain walls the energy is proportional to the domain wall length. If we assume that the domains run vertically though the entire ferroelectric then each domain wall has the same length and the domain wall energy becomes proportional to the number of domains which is inversely proportional to the domain width; $n=1 / w$. Therefore the energy associated with the domain walls is $E=\sigma d / w$ where $\sigma$ is the domain wall energy density, $d$ is the thickness of the ferroelectric, and $w$ is the domain width. Summing these two term and minimizing with respect to the domain wall width leads a square root dependence of the domain width with the ferroelectric thickness; $w=\sqrt{\sigma d / U}$. This simple model was originally developed for ferromagnetic domains [11] but is easily adapted to ferroelectric [12], ferroelastic [13] and ferroelectric/ferroelastic $[14,15]$ domains by choosing the energy densities corresponding to the electric and elastic effects. What is remarkable is that such a simple theory is able to predict the behavior of the domain wall width with thickness over six orders of magnitude [16].

Ferroelectrics domains are able to lower the free energy of the material by reducing the electrostatic field or the mechanical stress in the material. The boundary conditions are the electrical and mechanical environment which the ferroelectric is subjected and they determine the domain structure in the material. How the electrical and mechanical boundary conditions determine the domain structure is discussed in detail below.

In the bulk of a ferroelectric the charge separation in each unit cell created by the polarization is compensated by the opposite charge from the adjacent unit cells. However at the surface of the material this charge is uncompensated resulting in bound surface charges, Figure 2.3a. 
a)
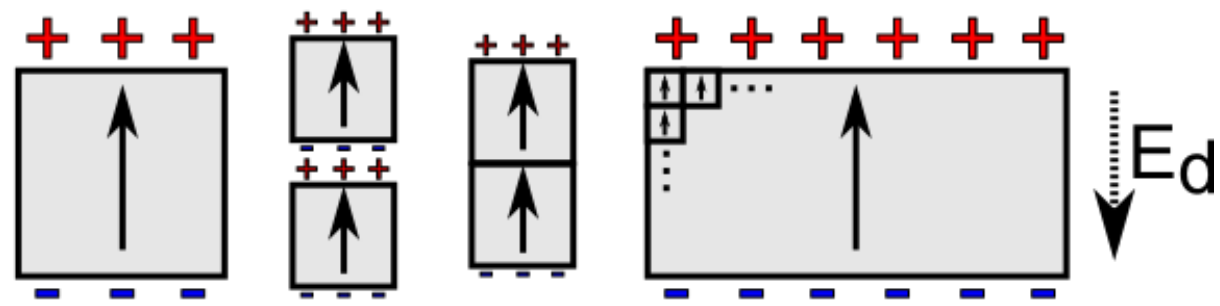

b)
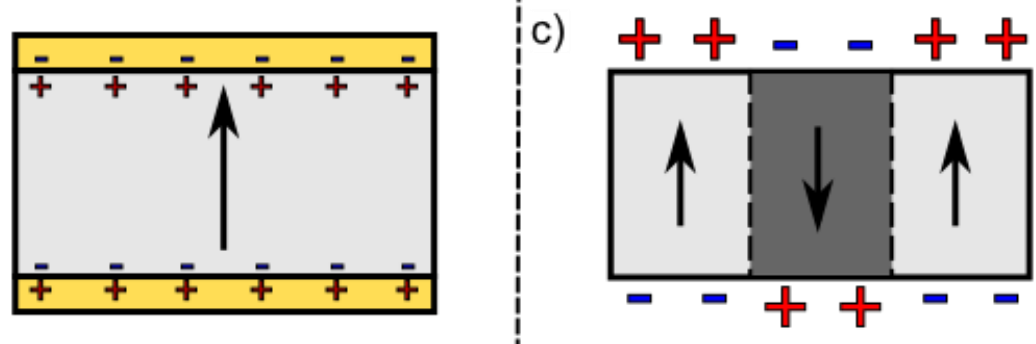

Figure 2.3 Origin of the electrical boundary condition for a ferroelectric, a) depolarizing field as a result of surface charges, b) screening of surface charges by electrodes, and c) reduction of the depolarizing field by forming $180^{\circ}$ domains.

These surface charges create an electric field in the material oriented in the opposite direction to the polarization call the depolarizing field. The name depolarizing field comes from the fact that this electric field opposes the polarization and if it is large enough will completely remove the polarization and the material will no longer be ferroelectric. The strength of the depolarizing field is inversely proportional to the thickness of the film and is a major problem when fabricating nanoscale ferroelectrics. However, the depolarizing field can be minimized in a number of ways. First electrodes can be placed at the surface of the ferroelectric and the surface charges in the ferroelectric are screened by the free charge carriers in the electrode preventing the depolarizing field, Figure 2.3b. Secondly, if no electrodes are present then polar molecules in the atmosphere will be attracted to the surface changes providing some screening reducing the depolarizing field. Finally, the ferroelectric can reduce the depolarizing field by forming $180^{\circ}$ domains, Figure $2.3 \mathrm{c}$. Now each surface will consist of alternating regions of positive and negative surface charges depending on the domain orientation in each region of the film. However, the net charge of the entire surface will be zero thereby reducing the depolarizing field.

Ferroelectric/ferroelastic domains are formed due to the mechanical boundary condition placed on a ferroelectric. For an isolated ferroelectric slab the material mechanically free and therefore under no strain. In practice ferroelectric are grown on a substrate material. If the growth is epitaxial then the crystal structure of the ferroelectric will conform to the 
structure of the underlying substrate. A difference in the lattice parameters and or the thermal expansion of the ferroelectric and substrate will introduce strain into the ferroelectric layer (assuming the substrate layer is much thicker than the ferroelectric). The ferroelectric can minimize this strain in two ways. First dislocations can form at the ferroelectric-substrate interface allowing the ferroelectric crystal to relax to its bulk lattice parameters. Alternatively the ferroelectric can rearrange part of its crystal structure to accommodate the strain. This process is demonstrated in Figure 2.4.
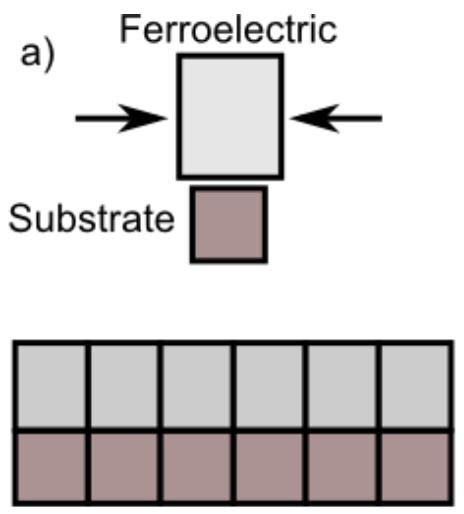

\section{Compressive Strian}

b)
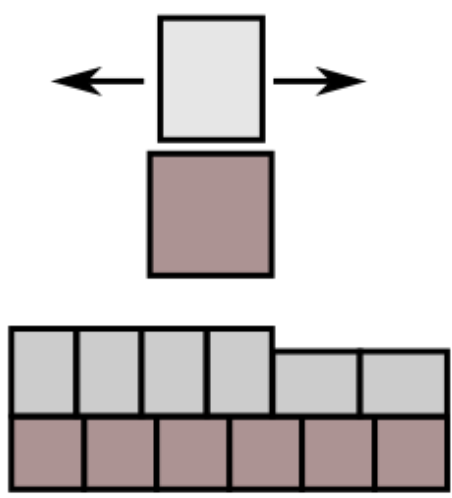

Small Tensile Strian

Figure 2.4 Mechanical boundary effects a) ferroelectric film under compressive strain and b) ferroelectric film under small tensile strain showing a $90^{\circ}$ domain.

Consider the case of a tetragonal ferroelectric that is under compressive strain, Figure $2.4 \mathrm{a}$, or tensile strain, Figure 2.4b. When the ferroelectric is under tensile strain then ferroelectric will have its long axis perpendicular to the interface therefore minimizing the difference in lattice parameters between the ferroelectric and substrate. Conversely, in the case of a large tensile strain then the entire film will have it long axis parallel to the interface. For small tensile strains, Figure $2.4 \mathrm{~b}$, the film will adopt a mixed state. In this way the ferroelectric can minimize its strain while still adopting the periodicity of the substrate crystal over a period of several unit cells.

\subsection{Domain walls}

In ferroelectrics, unlike ferromagnets, domain walls are atomically sharp extending for only one or two unit cells. The extremely small dimensions of ferroelectric domain walls might seem to make their properties irrelevant this is not necessarily true. The inverse square root law for domain width means that as the dimension of the ferroelectric are reduce the number of domains and hence domain walls increases. At sufficiently small dimensions the 
domain wall can account for a significant fraction of the ferroelectric. For example a $100 \mathrm{~nm}$ thick ferroelectric film could a volume fraction of domain walls between 6-20\% [17]. Therefore, the properties of domains can no longer be neglected and opens the possibility to use the properties of domain walls to either improve the bulk properties are fabricate devices where the domain walls are the active elements.

\subsubsection{Domain wall conductivity}

One of the most exciting and actively studied domain wall phenomena is domain wall conductivity in an insulating ferroelectric. One of the first reports of a change in the electronic properties of domain walls occurred with the discovery of superconductivity at ferroelastic domain walls in $\mathrm{WO}_{3}$ [18]. Research into ferroelectric domain wall conductivity exploded with the report of domain wall conductivity in written domains in $\mathrm{BiFeO}_{3}$ [19]. Since then domain wall conductivity has been reported in a wide range of ferroelectric domains including asgrown $\mathrm{BiFeO}_{3}$ domain wall [20, 21], $180^{\circ} \mathrm{PZT}$ domain walls [22], and even charged domain walls in $\mathrm{HoMnO}_{3}$ [23]. The difficulty in these studies is to determine the conduction mechanism taking place in the domain walls. Using conductive atomic force microscopy (described in Chapter 2) IV curves of the domain wall can be obtained and are fitted to theoretical behavior of different conduction mechanisms to determine the physical origin of the domain wall conductivity.

In general there are five conduction mechanisms can occur in a semiconducting material such as ferroelectrics. These five mechanism are divided into two groups, interface and bulk limited, depending on the where the largest obstacle for motion of the charge carrier. For interface limited conduction the biggest obstacle is injecting charge carriers into the ferroelectric from electrodes or vacuum. In bulk limited conduction charge carriers can easily enter the ferroelectric and the biggest obstacle is their flow through the material.

The two bulk limited conduction methods are spaced charged limited and PooleFrenkel hopping conduction. In space charged limited conduction charge is able to freely flow into the material and charge carriers accumulate at the interface of the ferroelectric. Electric fields generated by this buildup of charge will cause the charge carriers to diffuse through the material. Space charge limited conduction is proportional to the square of the voltage and inversely proportional to the length of the material cubed, $\mathrm{J} \alpha \mathrm{V}^{2} / \mathrm{L}^{3}$ [24]. In Poole-Frenkel conduction charge carriers are once again able to easily enter the ferroelectric. Once in the material charge carriers are able to hop between defect sites, which serve as local potential wells in the material, with the application of an external electric field. In this case $\log (\mathrm{J} / \mathrm{E}) \alpha$ $(\mathrm{E} / \mathrm{L})^{1 / 2}[24]$. 
There are three interface limited conduction methods; Schottky thermionic emission, Richardson-Schottky-Simmons emission, and Fowler-Nordheim tunneling. When a metal is placed in contact with a semiconductor a potential barrier will develop as the Fermi energy of the two materials align. This potential is known as the Schottky barrier and charge carriers entering the semiconductor must overcome this barrier by either applied bias or thermal excitation. As a result, the relationship between current, temperature and applied field is $\log \left(\mathrm{I} / \mathrm{T}^{2}\right) \propto \mathrm{E}^{1 / 2}$ [24]. Richardson-Schottky-Simmons emission is a special case of Schottky emission in the low mobility regime where the mean free path of the charge carriers is very short compared to the length of the material. In this case the $\log (\mathrm{I} / \mathrm{E}) \propto \mathrm{E}^{1 / 2}$ [25]. Finally in Fowler-Nordheim tunneling the charge carriers do not overcome the Schottky barrier but instead quantum mechanically tunnel through the barrier into the semiconductor. FowlerNordheim results in a $\log \left(\mathrm{I} / \mathrm{E}^{2}\right) \alpha-1 / \mathrm{E}$ dependence [26]. 


\section{References}

[1] J. Valasek, "Piezo-Electric Activity of Rochelle Salt under Various Conditions," Physical Review, vol. 19, pp. 478-491, 05/01/ 1922.

[2] A. von Hippel, R. G. Breckenridge, F. G. Chesley, and L. Tisza, "High dielectric constant ceramics," Industrial \& Engineering Chemistry, vol. 38, pp. 1097-1109, 1946/11/01 1946.

[3] W. B. and G. I.M., C. R. Acad. Sci. URSS, vol. 46, p. 139, 1946.

[4] J. F. Scott, "Applications of Modern Ferroelectrics," Science, vol. 315, pp. 954-959, February 16, 20072007.

[5] A. J. Lovinger, "Ferroelectric Polymers," Science, vol. 220, pp. 1115-1121, 19831983.

[6] Y. Liu, Y. Zhang, M.-J. Chow, Q. N. Chen, and J. Li, "Biological Ferroelectricity Uncovered in Aortic Walls by Piezoresponse Force Microscopy," Physical Review Letters, vol. 108, p. 078103, 02/13/ 2012.

[7] J. F. Scott and C. A. P. Dearaujo, "Ferroelectric Memories," Science, vol. 246, pp. 1400-1405, Dec 151989.

[8] M. Dawber, K. M. Rabe, and J. F. Scott, "Physics of thin-film ferroelectric oxides," Reviews of Modern Physics, vol. 77, pp. 1083-1130, Oct 2005.

[9] R. Waser, Nanoelectronics and Information Technology: Wiley, 2012.

[10] K. Uchino, Ferroelectric Devices: Marcel Dekker, 2000.

[11] C. Kittel, "Theory of the Structure of Ferromagnetic Domains in Films and Small Particles," Physical Review, vol. 70, pp. 965-971, 12/01/ 1946.

[12] T. Mitsui and J. Furuichi, "Domain Structure of Rochelle Salt and KH_\{2\}PO_\{4\}," Physical Review, vol. 90, pp. 193-202, 04/15/ 1953.

[13] A. L. Roitburd, "Equilibrium structure of epitaxial layers," physica status solidi (a), vol. 37, pp. 329-339, 1976.

[14] W. Pompe, X. Gong, Z. Suo, and J. S. Speck, "Elastic Energy-Release Due To Domain Formation In The Strained Epitaxy Of Ferroelectric And Ferroelastic Films," Journal of Applied Physics, vol. 74, pp. 6012-6019, Nov 1993.

[15] N. A. Pertsev and A. G. Zembilgotov, "Energetics And Geometry Of 90-Degrees Domain-Structures In Epitaxial Ferroelectric And Ferroelastic Films," Journal of Applied Physics, vol. 78, pp. 6170-6180, Nov 1995.

[16] G. Catalan, I. Lukyanchuk, A. Schilling, J. M. Gregg, and J. F. Scott, "Effect of wall thickness on the ferroelastic domain size of BaTiO3," Journal of Materials Science, vol. 44, pp. 5307-5311, 2009/10/01 2009.

[17] G. Catalan, J. Seidel, R. Ramesh, and J. F. Scott, "Domain wall nanoelectronics," Reviews of Modern Physics, vol. 84, pp. 119-156, 2012.

[18] A. Aird and E. K. H. Salje, "Sheet superconductivity in twin walls: experimental evidence of WO3-x," Journal of Physics-Condensed Matter, vol. 10, pp. L377-L380, Jun 8 1998. 
[19] J. Seidel, L. W. Martin, Q. He, Q. Zhan, Y. H. Chu, A. Rother, et al., "Conduction at domain walls in oxide multiferroics," Nat Mater, vol. 8, pp. 229-34, Mar 2009.

[20] J. Seidel, P. Maksymovych, Y. Batra, A. Katan, S. Y. Yang, Q. He, et al., "Domain Wall Conductivity in La-Doped BiFeO_\{3\}," Physical Review Letters, vol. 105, 2010.

[21] S. Farokhipoor and B. Noheda, "Conduction through $71^{\circ}$ Domain Walls in BiFeO_\{3\} Thin Films," Physical Review Letters, vol. 107, 2011.

[22] J. Guyonnet, I. Gaponenko, S. Gariglio, and P. Paruch, "Conduction at domain walls in insulating $\mathrm{Pb}(\mathrm{Zr0}$.2 Ti0.8)O3 thin films," Adv Mater, vol. 23, pp. 5377-82, Dec 1 2011.

[23] W. D. Wu, Y. Horibe, N. Lee, S. W. Cheong, and J. R. Guest, "Conduction of Topologically Protected Charged Ferroelectric Domain Walls," Physical Review Letters, vol. 108, Feb 2012.

[24] P. Zubko, D. J. Jung, and J. F. Scott, "Electrical characterization of PbZr0.4Ti0.6O3 capacitors," Journal of Applied Physics, vol. 100, pp. -, 2006.

[25] J. G. Simmons, "Richardson-Schottky Effect in Solids," Physical Review Letters, vol. 15, pp. 967-968, 12/20/ 1965.

[26] N. M. Ravindra and J. Zhao, "Fowler-Nordheim conduction in polysilicon (n[sup +])-oxide-silicon (p) structures: Limit of the classical treatment in the barrier height determination," Journal of Applied Physics, vol. 89, p. 7994, 2001. 


\section{Chapter 3}

\section{Materials and Methods}

\subsection{Introduction}

This chapter will discuss the experimental techniques used for the thin film growth and characterization for the study of ferroelectric size effects explored in this thesis. This chapter will provide both a theoretical background for the various experimental techniques as well as discuss the specific settings and conditions used for the growth and characterization of the samples studied. In addition, relevant information about the materials used for the study of ferroelectric size effects is also provided.

Pulsed Laser Deposition is used for the growth of the epitaxial oxide films investigated in this thesis. The specifics of this technique are discussed starting from the selection and treatment of the substrates to the details of how thin film growth takes place and the actual conditions used for the deposition of the materials. Scanning probe microscopy is used for the characterization of the nanoscale properties of the ferroelectric films and other objects explored in this thesis. Scanning probe microscopy encompasses a wide range of techniques and details on the theory and operation of Atomic Force Microscopy, Conducting Atomic Force Microscopy and Piezoresponse Force Microscopy which are the three techniques most used in this thesis are discussed.

\subsection{Epitaxial growth}

Epitaxial growth is the growth of one crystal, the epitaxial film, on another crystal, the substrate, where the film adopts at least one well defined orientation of its crystal lattice with respect to the substrate lattice. The perovskite family of crystals is an ideal system for epitaxial growth for a number of reasons. First, the versatility of elements that can be inserted into the perovskite crystal structure results in a perovskites with a wide variety of physical properties. Combining two or more perovskites with different properties can result in a new tailored material with properties not found in either of the starting materials. Secondly, all the perovskites share the same crystal structure making epitaxial growth much easier. 
For epitaxial growth to occur two elements are required; a high crystalline quality starting substrate and a growth technique capable of producing single crystalline films with few defects. Both of these requirements are discussed below starting with the preparation of single crystalline substrates

\subsubsection{Substrates thin film growth}

Throughout most of this thesis $\mathrm{DyScO}_{3}$ is used as a substrate. The crystal structure and lattice parameters of $\mathrm{DyScO}_{3}$ are given in Table 3.1. These polished substrates were obtained commercially from Crystec $\mathrm{GmbH}$. The physical dimensions of the $\mathrm{DyScO}_{3}$ substrate were $5 \mathrm{~mm}$ by $5 \mathrm{~mm}$ by $0.5 \mathrm{~mm}$ with a sample miscut of $<0.2^{\circ}$ with respect to crystal lattice. As received substrates must be cleaned and treated in order to obtain atomically smooth surfaces suitable for epitaxial growth. The treatment procedure used depends on the type of sample that would be grown. In all cases the substrate was first thoroughly cleaned first by ultrasonication for $20 \mathrm{~min}$ in acetone and then ethanol. Afterwards, the substrate surface was visibly inspected for contamination using an optical microscopy and any remaining dirt or dust was physically removed using lens paper in ethanol. The $\mathrm{DyScO}_{3}$ was then annealed to create well defined atomically smooth surface steps. The substrates were annealed for four hours at $1000^{\circ} \mathrm{C}$ with a $150 \mathrm{ml} / \mathrm{min} \mathrm{O}_{2}$ flow. In Chapter 5 and $6 \mathrm{SrRuO}_{3}$ nanowires were grown on mixed terminated $\mathrm{DyScO}_{3}$ surface and no further treatment was performed. In Chapter 4 a complete $\mathrm{SrRuO}_{3}$ films was grown and the $\mathrm{DyScO}_{3}$ was chemically treated in order to obtain a single $\mathrm{ScO}_{2}$ surface termination [1]. In this case the substrate was etched in $12 \mathrm{M} \mathrm{NaOH}$ for two hours followed by 1 hour in $1 \mathrm{M} \mathrm{NaOH}$.

In Chapter 6 lateral ferroelectric size effects were investigated by using single $\mathrm{Ca}_{2} \mathrm{Nb}_{3} \mathrm{O}_{10}$ nanosheets on silicon as a starting substrate. Here the silicon layer is a mechanical support for the $\mathrm{CaNb}_{3} \mathrm{O}_{10}$ nanosheets. The native silicon dioxide layer present at the surface is amorphous preventing epitaxial growth directly on the silicon substrate. The lattice constants of the $\mathrm{CaNb}_{3} \mathrm{O}_{10}$ nanosheets are given in Table 3.1. These single layer nanosheets were prepared in solution by exfoliation from parent compounds. Once exfoliated the nanosheets were deposited on silicon substrates using Langmuir-Blodgett deposition [2]. 


\begin{tabular}{|c|c|c|}
\hline Material & Crystal Structure & Lattice Parameter $(\AA)$ \\
\hline $\mathrm{DyScO}_{3}$ & orthorhombic & $a_{p c}=3.95$ \\
\hline $\mathrm{Ca}_{2} \mathrm{Nb}_{3} \mathrm{O}_{10}$ & cubic & $\mathrm{a}=\mathrm{b}=\mathrm{c}=3.86$ \\
\hline $\mathrm{SrRuO}_{3}$ & orthorhombic & $a_{p c}=3.93$ \\
\hline $\mathrm{PbTiO}_{3}$ & tetragonal & $\mathrm{a}=\mathrm{b}=3.904 \mathrm{c}=4.152$ \\
\hline $\mathrm{BiFeO}_{3}$ & rhombohedral & $a=b=c=3.965$ \\
\hline
\end{tabular}

Table 3.1 List of materials used in this thesis with their room temperature crystal structure and lattice parameters. Note $a_{p c}$ is the psuedocubic lattice parameter

The prepared substrates were glued using silver paste onto sample plates for growth and in situ characterization. For electrical contact for Piezoresponse Force Microscopy measurements a small amount of silver paste was applied to top surface in one of the corners of the substrate. The glued substrates were heated to $\sim 200^{\circ} \mathrm{C}$ for $5 \mathrm{~min}$ in order to dry and cure the silver paste. The glued substrate was then loaded into a UHV distribution chamber where the sample would be clamped onto a heater for subsequent thin film growth by Pulsed Laser Deposition.

\subsubsection{Pulsed laser deposition}

Pulsed Laser Deposition is a thin film growth technique that has become popular in the oxide community for the growth of a wide range of single crystalline oxide materials. Although PLD was first developed in the 1960's [3] is wasn't until the late 80's that PLD became a common growth technique after PLD was used to deposit high temperature superconductor $\mathrm{YBa}_{2} \mathrm{Cu}_{3} \mathrm{O}_{7}$ thin films with superior properties compared to other growth techniques[4]. The development of in situ growth monitoring using Reflection High Energy Electron Diffraction allows the growth process of the film to be precisely controlled with single monolayer precision [5]. PLD has a number of advantages over other growth technique that make it a popular choice for researchers. First, the growth is fast compared to other techniques and second stoichiometric transfer of complex materials from target to substrate is much easier [6].

A schematic of the experimental setup for PLD growth is shown in Figure 3.1 and details of the experimental settings used for the deposition of the various materials are shown in Table 3.2. 


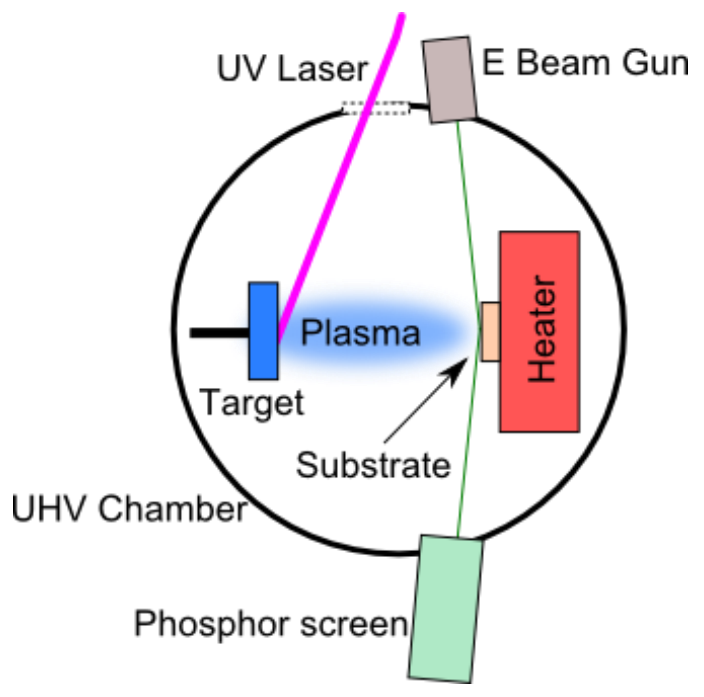

Figure 3.1 Schematic of pulsed laser deposition showing the major components involved in the process

In this thesis a PLD system from Twente Solid State Technology was used. The process takes place in a UHV chamber with a background pressure of $10^{-8}$ mbar. Typically a small amount of gas, usually oxygen and or argon, is let into the deposition chamber serving two functions; to control the kinetic energy of the plasma species when they arrive at the substrate surface, and with oxygen, to oxidize the plasma for the reduction of oxygen vacancies and other defects in the film. Furthermore, after depositions the films are annealed in high oxygen pressure $(100 \mathrm{mbar})$ by slow cooling $\left(-5^{\circ} \mathrm{C} / \mathrm{min}\right)$ back to room temperature to further improve the crystalline quality and minimize the oxygen vacancies.

\begin{tabular}{|l|c|c|c|c|c|}
\hline & $\begin{array}{c}\mathrm{SrRuO}_{3} \\
\text { (film) }\end{array}$ & $\begin{array}{c}\mathrm{SrRuO}_{3} \\
\text { (wires) }\end{array}$ & $\mathrm{SrTiO}_{3}$ & $\mathbf{P b T i O}_{3}$ & $\mathrm{BiFeO}_{3}$ \\
\hline $\begin{array}{l}\text { Energy Density } \\
\left(\mathrm{J} / \mathrm{cm}^{2} \text { ) }\right.\end{array}$ & 2.5 & 2.1 & 1.3 & 2.0 & 1.9 \\
\hline Temperature ( $\left.{ }^{\circ} \mathbf{C}\right)$ & 630 & 630 & 670 & 610 & 630 \\
\hline Process Gas & $\mathrm{O}_{2}$ & $\mathrm{O}_{2} / \mathrm{Ar}(5050)$ & $\mathrm{O}_{2}$ & $\mathrm{O}_{2}$ & $\mathrm{O}_{2}$ \\
\hline $\begin{array}{l}\text { Process Pressure } \\
\text { (mbar) }\end{array}$ & 0.06 & 0.3 & 0.01 & 0.13 & 0.3 \\
\hline $\begin{array}{l}\text { Laser Frequency } \\
\text { (Hz) }\end{array}$ & 1 & 1 & 1 & 1 & 4 \\
\hline
\end{tabular}

Table 3.2 Deposition parameters for the materials used in this thesis. For all depositions the spot size was $2.3 \mathrm{~mm}^{2}$ and the samples were annealed by slow cooling to room temperature at $-5^{\circ} \mathrm{C} / \mathrm{min}$ in $100 \mathrm{mbar} \mathrm{O}_{2}$. 
A UV laser with $\lambda=248 \mathrm{~nm}$ and a pulse duration of $25 \mathrm{~ns}$ is focused onto a ceramic target of the material to be deposited. A rectangular mask and several lenses in the UV laser beam path focus the laser on the target providing a well-defined homogeneous laser spot of 2.3 $\mathrm{mm}^{2}$. The laser energy was controlled using an attenuator in the beam path. When a laser pulse with sufficient energy initially hits the surface of the target the material ablates causing a plasma to form at the immediate surface of the target. As the remainder of the laser pulse transfers energy into the plasma it will heat and expand, moving away from the target surface and travels to the substrate placed parallel to the target. It is important the laser provides enough energy to ablate the target material instead of evaporation. If material evaporates then the concentration of the target species leaving the target surface depends on the volatility of the various elements. Instead, with ablation all the elements are vaporized from the target regardless of their volatility making stoichiometric transfer much more likely.

The substrate is heated to improve the growth of the material once the plasma species have arrived on the surface. Two heating methods were used during the PLD growth in this thesis; resistive heating and laser heating. In resistive heating a current is passed through a spiraled wire in the heater and provided accurate temperature control up to $850^{\circ} \mathrm{C}$. A thermocouple in the heating block provided temperature control; however, a pyrometer was used to accurately check the temperature on the substrate surface which could vary by as much as $50^{\circ} \mathrm{C}$ from the thermocouple reading. For laser heating an infrared diode laser was focused on the backside of a sample plate containing the glued substrate. The temperature of the substrate was controlled using a pyrometer aimed at the sample surface. The pyrometer was first calibrated using a silicon sample; a material where the emissivity is well known [7]. Laser heating provides a number of advantages over resistive heating. Since only the sample plate is heated using laser heating as opposed to an entire heating block in resistive heating the temperature can be changed much faster and contamination due to evaporation from material from pervious deposition is greatly reduced.

The film growth is monitored in situ using RHEED. This diffraction technique uses an electron beam positioned at grazing incidence to the sample surface. The resulting specular and diffracted beams are detected using a phosphor screen and a CCD camera. When the incident electrons hit the sample surface they will diffracted by crystal structure of only topmost $10-100 \AA$ of material due to the grazing angle of the beam with respect to the substrate surface. As a result, RHEED is a very surface sensitive technique which makes it well suited to monitor monolayer by monolayer growth of a film. The diffraction spots will appear lie on a circle due to the $2 \mathrm{D}$ nature of the sample surface and provide information on the crystal structure from the spacing of the spots. The specular spot is the mirror-like reflection of the electrons from the sample surface and the intensity of the spot provides real-time information of the progress of the film growth. Surface steps will cause the electrons from the specular reflection to scatter and therefore the intensity of the specular spot is proportional to the surface step density. During layer by layer growth the surface starts as an atomically smooth 
substrate with only vicinal steps due to the miscut. This represents the maximum intensity of the specular spot. Each laser pulse deposits a fraction of a monolayer of material. As nucleation and growth takes place the surface step density increases causing a decrease in the specular spot intensity. This trend continues until a half monolayer of material is deposited. Now, further material arriving at the surface will fill in the reaming gaps in the monolayer coverage resulting in a decrease in the surface step density and an increase in the intensity. Once the monolayer is complete the only steps present are the vicinal steps from the miscut and the specular spot intensity returns to its maximum intensity. By counting the intensity oscillations the thickness of the film is controlled with single monolayer precision. In this thesis RHEED was used to determine the thickness of the PLD grown films by monitoring the intensity of the specular spot. This was accomplished using a Staib Instruments RHEED setup using a $30 \mathrm{keV}$ operating voltage.

\subsection{Scanning probe microscopy}

Scanning Probe Microscopy refers to a family of experimental techniques which characterize the surface a material using an atomically sharp tip that is scanned over the surface. The specific techniques in this family are defined by the nature of the excitation and feedback used to explore the surface. The field of scanning probe microscopy began in 1981 with the development of Scanning Tunneling Microscopy for imaging the surface topography of conducting materials on the atomic scale [8] and its use to determine the structure of the surface of silicon made the technique a huge success [9]. In STM the tunneling current is measured between an atomically sharp tip and a conducting surface for a given tip position and applied bias. The tunneling current depends exponentially on distance between the tip and surface and by maintaining a constant tunneling current the surface topography of the sample can be traced by scanning the tip over the surface. The ability of STM to provide atomic resolution made an instant impact in the scientific community eventually winning its developers the Nobel Prize.

The limitation of only being able in investigate the surface on conducting and semiconducting materials lead to the development of atomic force microscopy in 1986 which could provide atomically resolved surface topography information from any material including insulators [10]. Instead of using current to maintain a small tip-surface separation the AFM uses force between a surface and an atomically sharp tip on a cantilever to probe a surface. From here the floodgates to scanning probe microscopy were opened and many new techniques based off either STM or AFM were developed to provide highly resolved surface maps of many properties including conductivity, magnetism, surface potential, electrical polarization, and many more. In this thesis atomic force microscopy, conducting atomic force microscopy, and piezoresponse force microscopy are used to investigate the surface 
topography, conductivity and polarization of ferroelectric films and objects. The operational principles and imaging conditions for these three techniques are described below

\subsubsection{Atomic force microscopy}

In Atomic Force Microscopy the surface topography is imaged by an atomically sharp tip on a cantilever which detects forces between the tip and a sample surface. A typical AFM setup is shown in Figure 3.2a and consists of five basic components; a cantilever with an sharp tip having a radius of 5-10 nm, a laser, a four quadrant photodetector, a XYZ piezoscanner, and a feedback control mechanism.

The first step in any AFM measurement is to bring the tip and sample close together through a combination of a stepper motor for coarse motion and a piezoelectric scanner which provides subnanometer movement. As the tip is brought in close proximity to the sample different forces will cause an interaction between the surface and the tip. The forces acting between the tip and sample are modeled using the Lennard-Jones potential, given by $F(r)=$ $-\frac{6 A}{r^{7}}+\frac{12 B}{r^{13}}$ shown in Figure 3.2b [11]. At "large" distances, less than $10 \mathrm{~nm}$, attractive Van der Waal forces dominate, given by the $-\frac{6 A}{r^{7}}$ term. As the tip sample distance decreases to $<1 \AA$ electrostatic repulsion between overlapping electron clouds from the atoms of the tip and surface come into effect. This results in strong repulsive force acting on the tip, given by the $\frac{12 B}{r^{13}}$ term, which rapidly overwhelms the weaker attractive interactions.

When the tip is scanned across the sample surface changes in the topography will change the tip sample distance and as a result the force acting on the tip will also change. The cantilever will therefore be deformed from its initial position causing a movement of the cantilever perpendicular to the surface. By detecting this motion the tip-surface distance can be adjusted by the $\mathrm{Z}$ piezo to maintain a constant force and by keeping track of the changes in height needed to maintain a constant force the surface topography is recorded as the tip is scanned across the surface. Detection of the cantilever motion can be accomplished by several methods. The most common method and the one used for all AFM based measurements in this thesis is the beam defection method. Here a reflective coating is applied to the top side of the cantilever and a laser beam is positioned onto the end of the cantilever so the beam reflects onto a four quadrant photodetector. As the cantilever bends up and down the position of the laser spot on the photodetector will also move up and down. The change in laser intensity between the four quadrants on the photodetector is then used as the feedback mechanism for the $\mathrm{Z}$ piezo to maintain a constant force between the tip and surface.

The specifics of how the tip surface forces are used to image the surface topography depends on the AFM mode being used. For AFM topography imaging the two most common methods 
are contact mode and tapping mode. In contact mode the tip is brought into "contact" with the surface where the repulsive electrostatic interactions dominate. The sample is then scanned across the surface and feedback from the photodetector is used to maintain a constant force to image the surface topography. Contact mode provides a fundamentally simple means to image a sample surface with easy data interpretation. However, with the tip inconstant contact with the surface tip wear and damage can result in a loss of resolution. Contact mode AFM is also not possible for soft samples but this is not an issue for the materials studied in this thesis. In tapping mode AFM the cantilever is brought into close contact with the surface and driven to oscillate at or near its resonance frequency by a piezoactuator. When the tip and sample distance changes the change in force interacting between the tip and surface will cause a shift in the resonance frequency, show in Figure 3.2c. Changes in cantilever resonance can then be detected by two means, either by amplitude detection (blue lines) or by frequency detection (red lines). In either case, by adjusting the $\mathrm{Z}$ piezo the resonance is shifted back and the height change needed is recorded to provide measurement of the surface topography. The main advantage of tapping mode is that the tip spends much less time in contact with the surface, only once per oscillation, reducing tip damage and wear and making it possible to image soft samples.

a)

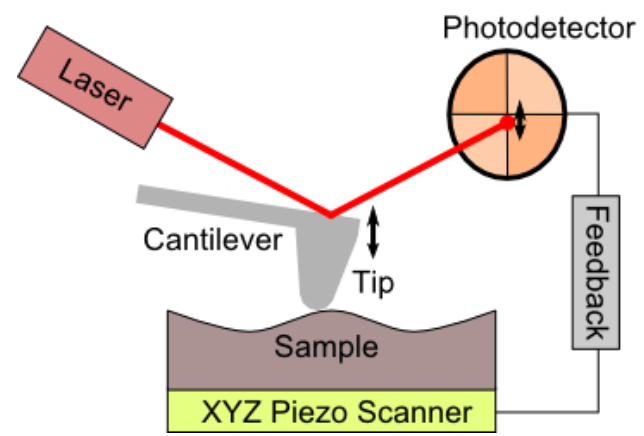

b)

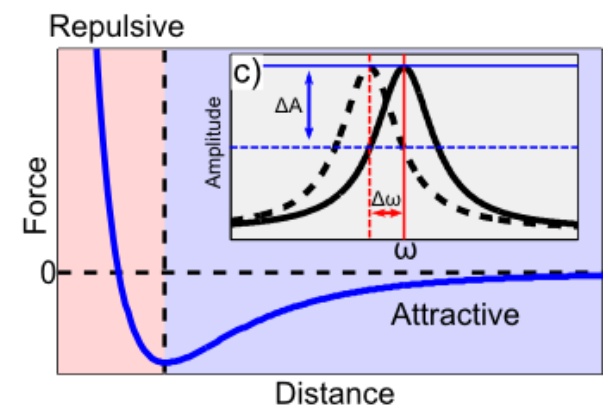

Figure 3.2 a) Schematic of contact mode AFM using beam deflection detection scheme. b) Lennard-Jones potential showing the two force regimes acting on the tip, c) Frequency response of a cantilever during tapping mode AFM and a shift in the resonance (black dashed line) due to a change in the tip-sample distance and the two detection methods to monitor the change in resonance, amplitude detection (blue lines) and frequency detection (red lines).

In this thesis tapping mode AFM was used for imaging the topography of substrates and samples, for example Figure 7.2. Contact mode AFM was used for advanced AFM techniques, cAFM and PFM discussed below, while simultaneous imaging the topography. TESP tapping mode tips, $320 \mathrm{kHz}$ resonance frequency $42 \mathrm{~N} / \mathrm{m}$ spring constant, from Bruker Nano were used for topography imaging. Contact mode measurements were performed using 
ElectriMulti75-G tips from Budget Sensors, $3 \mathrm{~N} / \mathrm{m}$ spring constant $\mathrm{Cr} / \mathrm{Pt}$ conductive coating, for cAFM and PFM experiments. A Bruker Dimension Icon AFM was used for the ex situ AFM measurements and an Omicron UHV variable temperature AFM/STM was used for in situ scanning probe measurements which has been modified to be able to perform cAFM and PFM measurements.

\subsubsection{Conducting atomic force microscopy}

Conducting AFM is a contact mode technique used to simultaneously image the surface topography and sample conductivity and is used in Chapter 5 to investigate the conductivity of domain walls in $\mathrm{BiFeO}_{3}$. Shown schematically in Figure 3.3, the surface topography imaging and feedback control work in the exact same manner as a contact mode AFM experiment. What is different is that a voltage is applied between a conductive tip and the sample stage. The conductive tip acts as a local movable top electrode and the applied bias will result in a local current flow through the sample which is recorded using a current amplifier in series with the voltage source. Depending on the quality of the current amplifier, currents down to a few fA can be measured. Unlike STM, cAFM does not use the current as a feedback mechanism and as a result the conductivity maps can be measured on samples containing insulating and conductive regions while simultaneously recording the surface topography. The conductive tips with $\mathrm{Cr} / \mathrm{Pt}$ coating have a larger radius than standard contact mode tips, $25 \mathrm{~nm}$ as opposed to $12 \mathrm{~nm}$, so the minimum resolution is not as small for cAFM compared to contact mode AFM. However, cAFM still provides a lateral resolution of $\sim 10-30$ $\mathrm{nm}$ giving nanoscale information on the conductivity of a sample.

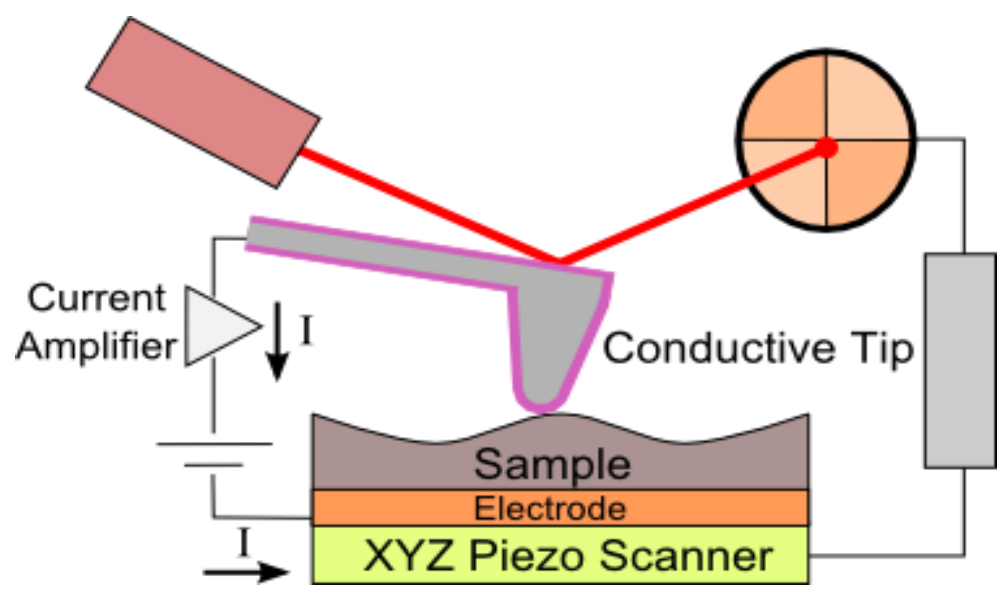

Figure 3.3 Schematic of a $A$ AFM experiment showing the major components of the technique. 


\subsubsection{Piezoresponse force microscopy}

Piezoresponse force microscopy as the name implies measures the piezoresponse from a piezoelectric or ferroelectric material. Since its development in the early 90's PFM has become the most widely used technique to locally probe the domain structure of ferroelectrics. PFM is a much more powerful tool than just an imaging technique. Along with just domain imaging it is also possible to pattern new domains and obtain local hysteresis loops. As a result PFM is ubiquitous with research into nanoscale ferroelectrics as it can provide nanoscale information on the polarization amplitude and orientation of domains as well as investigation of domain dynamics during switching. In this thesis PFM is used in Chapters $4,5,6$, and 7 to image the domain structure of the $\mathrm{PbTiO}_{3}$ and $\mathrm{BiFeO}_{3}$ films and relate the domain structure of the ferroelectrics to the topography and or conductivity. This specific settings used varied from sample to sample and were optimized for each measurement to obtain the best images but in general a probing voltage of $<2 \mathrm{~V}$ with an AC frequency of $<20 \mathrm{kHz}$ was used.

PFM is based on contact mode AFM and is shown schematically in Figure 3.4. As with cAFM, PFM uses a conductive tip as a local mobile top electrode to apply a bias between the tip and the sample stage. The operating principle behind PFM is the converse piezoelectric effect; $x_{j k}=d_{i j k} E_{i}$ where $\mathrm{x}_{\mathrm{jk}}$ is the strain tensor, $\mathrm{E}_{\mathrm{i}}$ is the electric field, and $\mathrm{d}_{\mathrm{ijk}}$ is a tensor that describes the magnitude of the piezoresponse [12]. Stated in words, when an electric field is placed across a piezoelectric material a deformation will occur. The deformation along the field direction is the longitudinal response, the deformation perpendicular to the field is the transverse response, and the deformations in other directions are shear piezoresponse. What makes the converse piezoelectric effect useful for domain imaging in ferroelectrics is that the direction of the deformation, expansion or contraction, is related to the orientation of the polarization with respect to the applied field. Consider the two cases in Figure 3.4a. The difference between the left and right schematics is the direction of the applied bias and hence the direction of the applied field in the ferroelectric. When the applied field is oriented in the same direction as the polarization the converse piezoelectric effect will result in an expansion of the material and vice versa. In-plane polarization is also detectable using PFM. In this case, shown in Figure 3.4b, transverse piezoresponse results in torsion of the cantilever instead of a vertical displacement. It is only possible to measure in-plane polarization that is perpendicular to the cantilever orientation. Piezoresponse along the cantilever direction will not be able to cause a torsion of the tip and in some cases will result in buckling of the cantilever which will show up as weak out of plane piezoresponse. To fully investigate in-plane response two measurements must be performed in the same location rotating the sample by $90^{\circ}$ after the first image. 
a)
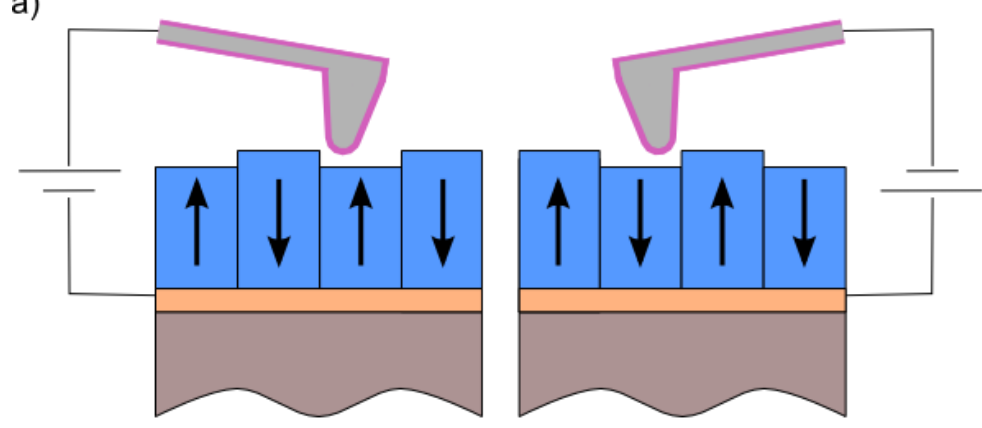

b)
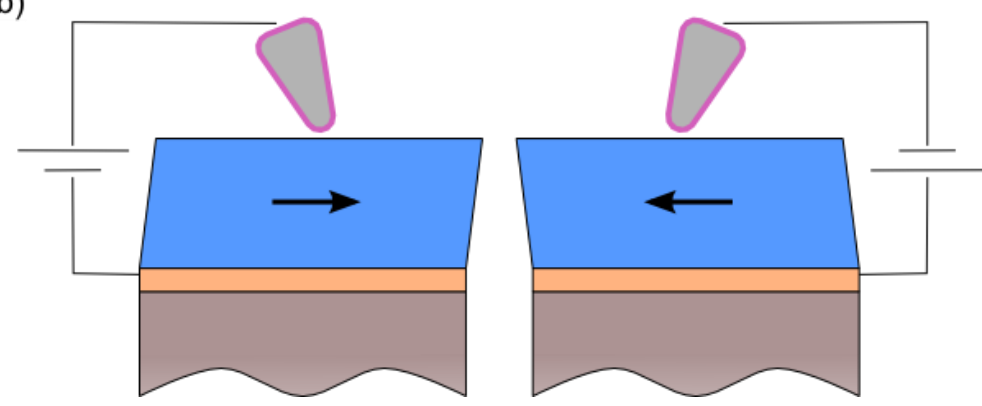

Figure 3.4 Schematic of a PFM experiment a) out of plane piezoresponse to an applied DC voltage of opposite polarity. b) in-plane piezoresponse to an applied DC voltage of opposite polarity showing the torsion of the tip.

For typical ferroelectrics the deformation, $\Delta \mathrm{z}=-\mathrm{d}_{33} \mathrm{~V} / \Delta \mathrm{x}=-\mathrm{d}_{15} \mathrm{~V}$, caused by the converse piezoelectric effect is very small $\sim 0.2 \mathrm{~nm}$. Therefore detecting these small changes can be challenging especially if there is also surface roughness. To get around this problem an AC voltage is applied and the piezoresponse is detected using a lock-in amplifier. By applying an AC voltage the deformation of the ferroelectric from the converse piezoresponse is now $\mathrm{z}(\mathrm{t})=-\mathrm{d}_{33} \sin (\omega \mathrm{t}+\theta) / \mathrm{x}(\mathrm{t})=-\mathrm{d}_{15} \sin (\omega \mathrm{t}+\theta)$ for the out of plane and in-plane response respectively where $\omega$ is the frequency of the AC voltage and $\theta$ is the phase of the response with respect to the AC voltage. Now information on the polarization orientation is given by the phase of the response. If the polarization is allied in the same direction as the applied field then the phase of the piezoresponse is $\theta=0^{\circ}$. When the polarization is antiparallel to the applied field the phase of piezoresponse is $\theta=180^{\circ}$. In most PFM experiments the piezoresponse is broken into its constituent components, the amplitude (A) and the phase response $(\theta)$. The phase response provides information on the polarization orientation while the amplitude is related to the piezoelectric coefficient. 
It is important to note that when a biased tip is brought in contact with a material the piezoresponse is not the only effect which acts on the cantilever. Electrostatic and dielectric effects will also be acting on the cantilever and these can interfere with the measurement creating anomalous results. However, these effects can be minimized by using appropriate tips and measurement conditions. Modeling of the contact mechanics of PFM has shown that the electrostatic contributions are minimized when using stiff tips where $\mathrm{k}>1$ and high contact forces $>10 \mathrm{nN}$ [13]. As stated earlier, the tips used for the PFM measurements in this thesis has a spring constant of $\mathrm{k}=3 \mathrm{~N} / \mathrm{m}$ and using strong contact forces $<10 \mathrm{nN}$. 


\section{References}

[1] J. E. Kleibeuker, G. Koster, W. Siemons, D. Dubbink, B. Kuiper, J. L. Blok, et al., "Atomically Defined Rare-Earth Scandate Crystal Surfaces," Advanced Functional Materials, vol. 20, pp. 3490-3496, 2010.

[2] M. Nijland, S. Kumar, R. Lubbers, D. H. Blank, G. Rijnders, G. Koster, et al., "Local Control over Nucleation of Epitaxial Thin Films by Seed Layers of Inorganic Nanosheets," ACS applied materials \& interfaces, 2014.

[3] H. M. Smith and A. F. Turner, "Vacuum Deposited Thin Films Using a Ruby Laser," Applied Optics, vol. 4, pp. 147-148, 1965/01/01 1965.

[4] D. Dijkkamp, T. Venkatesan, X. D. Wu, S. A. Shaheen, N. Jisrawi, Y. H. Min-Lee, et al., "Preparation of $\mathrm{Y}-\mathrm{Ba}-\mathrm{Cu}$ oxide superconductor thin films using pulsed laser evaporation from high Tc bulk material," Applied Physics Letters, vol. 51, pp. 619-621, 1987.

[5] G. J. H. M. Rijnders, G. Koster, D. H. A. Blank, and H. Rogalla, "In situ monitoring during pulsed laser deposition of complex oxides using reflection high energy electron diffraction under high oxygen pressure," Applied Physics Letters, vol. 70, pp. 1888-1890, 1997.

[6] R. Eason, Pulsed laser deposition of thin films: applications-led growth of functional materials: John Wiley \& Sons, 2007.

[7] R. Groenen, "Title," unpublished|.

[8] G. Binnig, H. Rohrer, C. Gerber, and E. Weibel, "Surface Studies by Scanning Tunneling Microscopy," Physical Review Letters, vol. 49, pp. 57-61, 07/05/ 1982.

[9] G. Binnig, H. Rohrer, C. Gerber, and E. Weibel, "7 $\times 7$ Reconstruction on Si(111) Resolved in Real Space," Physical Review Letters, vol. 50, pp. 120-123, 01/10/ 1983.

[10] G. Binnig, C. F. Quate, and C. Gerber, "Atomic force microscope," Physical review letters, vol. 56, p. 930, 1986.

[11] S. M. Salapaka and M. V. Salapaka, "Scanning Probe Microscopy," Control Systems, IEEE, vol. 28, pp. 65-83, 2008.

[12] A. Gruverman and S. V. Kalinin, "Piezoresponse force microscopy and recent advances in nanoscale studies of ferroelectrics," Journal of materials science, vol. 41, pp. 107-116, 2006.

[13] S. V. Kalinin and D. A. Bonnell, "Imaging mechanism of piezoresponse force microscopy of ferroelectric surfaces," Physical Review B, vol. 65, p. 125408, 2002. 


\section{Chapter 4}

\section{Piezoresponse Force}

\section{Microscopy in Ultra High}

\section{Vacuum}

\subsection{Introduction}

The development of many electronic devices utilizing ferroelectric components and the continuing drive for miniaturization of these devices require a characterization technique that is able to probe local ferroelectric properties on the nanoscale. This desire to nondestructively probe the local ferroelectric properties of a material led to the creation of Piezoresponse Force Microscopy (PFM) in the mid-1990s. This scanning probe technique takes advantage of the converse piezoelectric effect using an electrically biased conducting nanometer sized tip to create local electric fields and simultaneously measure the local electromechanical response of the material. More details on the operation of PFM are found in Chapter 2. PFM has rapidly became the most widely used technique to study local ferroelectric properties and has successfully been used to characterize all sorts of ferroelectric materials including thin films [1] and ceramics [2] as well as ferroelectric polymers and living tissue $[3,4]$.

Determination of the resolution of any characterization technique is important to establish the appropriate conditions under which useful information can be obtained from a measurement. Intuitively the concept of resolution is easy to understand, what is the smallest possible feature that can be measured by a given characterization technique. However, a mathematical rigorous definition of resolution is not as straight forward. The concept of resolution was first explored in the field of optical microscopy giving rise to the famous Rayleigh criterion. Rayleigh stated that two point sources are resolved if the central maximum of the diffraction pattern of one source is located at the first minimum in the diffraction pattern of the second point source and vice versa [5]. The Raleigh criterion is illustrated in 
Figure 4.1a where two point sources (dashed lines) and the combined intensity (solid lines) of two point sources are shown at three different separations from fully resolved, to just resolved, to unresolved. The Raleigh criterion is not absolute; two point sources might still be distinguishable even at distances less than the Raleigh criterion as long as there is still a central dip in the combined intensity distribution which has led to many alternate proposed criterion [6]. In scanning probe microscopy a different definition of resolution needs to be defined as there are no point scatterers in SPM techniques. Instead, in SPM the response signal changes continuously from one value to another as the atomically sharp probe scans over the surface. As a result the $25 \%-75 \%$ criterion is generally used to define the resolution of the measurement. This definition of the resolution is shown in Figure 4.1b. The 25\%-75\% criterion is defined as the distance the signal needs to change from $25 \%$ to $75 \%$ between two constant signal regions. Again, this definition is arbitrary and is used as a conservative measure of the resolution in SPM as smaller spatial changes in the signal can still be distinguished.

a)

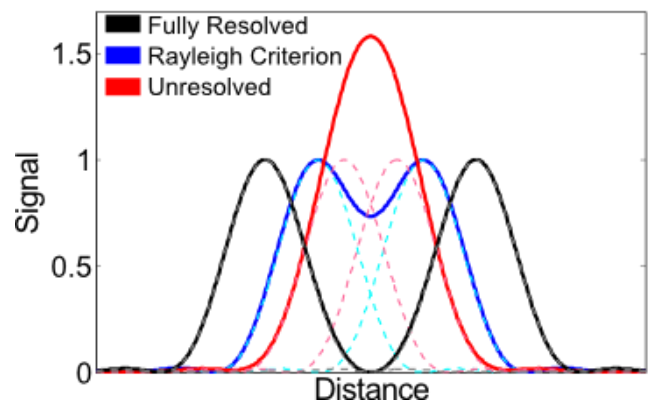

b)

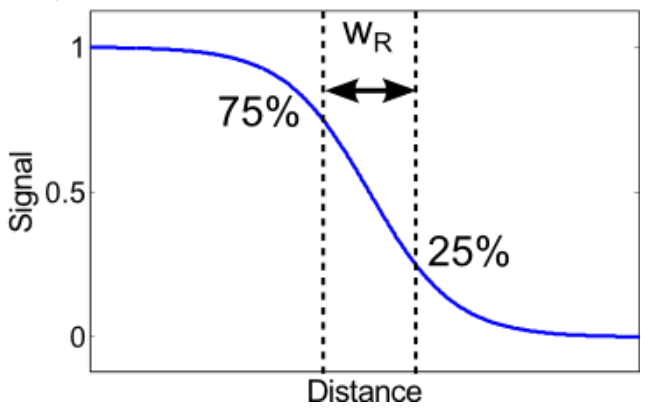

Figure 4.1 Schematic of the a) Raleigh criterion and b) 25-75 rule.

Based on the results of the many ferroelectric domain studies characterized by PFM the spatial resolution of the polarization orientation determined by PFM is in the nanometer regime. However, it is still important to establish the ultimate PFM resolution for this technique. This is an important question for the study of some ferroelectric systems where domain sizes are predicted to have a width of only a few nanometers such as the adaptive phase near the morphotropic phase boundary in PZT and certain nano-polar regions in relaxor ferroelectrics. Several studies, both experimental and theoretical, have been devoted to determining resolution of the PFM.

Experimentally the resolution of the PFM technique was explored by Kalinin et. al. in 2006 [7]. Using a PZT thin film on a $\mathrm{SrRuO}_{3}$ bottom electrode the PFM resolution was determined from diffractograms of periodically written domains and the measured width of a naturally occurring $180^{\circ}$ domain walls. The difractogram study consisted of writing a checkerboard pattern of alternating up and down $180^{\circ}$ domains. The checkerboard pattern 
was then imaged using PFM and the intensity of the peaks in Fourier transform was analyzed to determine a resolution of $82 \mathrm{~nm}$. Alternatively, a profile of the piezoresponse across one of the domain walls was extracted and using the 25-75 rule resulting in a resolution of $16 \mathrm{~nm}$. The large difference in these two resolutions was attributed to the difference between a readwrite process for the diffractogram approach and a read only process for the domain wall approach. Furthermore the resolution from the phase response profile across a $180^{\circ}$ domain wall as also determined and found to be 0.5 to 1 order of magnitude lower than the resolution from the full piezoresponse across a $180^{\circ}$ domain wall. This reduction is due to the limiting value of the phase response which can only take values of $0^{\circ}$ and $180^{\circ}$. However, the improved resolution comes at a price as the phase response does not contain all of the information of the piezoresponse and the phase resolution is thought of as the information limit of the PFM technique.

Theoretically, Morozovska et. al. developed analytical expressions for the resolution of the PFM in terms of tip geometry and materials parameters [8]. The contrast formation in PFM was modeled by determining the electroelasitic fields inside the ferroelectric material using a decoupled approach. With this approach first the electric field in the material due to a biased conducting tip is calculated using a rigid electrostatic model. Then the strain or stress field is calculated using the constitutive relations for a piezoelectric solid. Finally the resulting displacement field is determined using Green's functions. As a result the vertical PFM signal can be written as an expression for the spatial distribution of the material parameters and the tip parameters. Using this approach the functional dependence of the resolution was determined to be $r_{\min } \sim \frac{\varepsilon_{e} R_{0}}{\varepsilon_{11}}$ where $\mathrm{R}_{0}$ is the tip radius, $\varepsilon_{\mathrm{e}}$ is the external permittivity and $\varepsilon_{11}$ is the dielectric constant of the material. Therefore the highest resolution is obtained by PFM in vacuum with atomically sharp tips.

What is missing in these studies is a direct experimental determination of the PFM resolution under different conditions. Therefore, this chapter analyzes the PFM resolution from a $\mathrm{PbTiO}_{3}$ thin film in situ in UHV and in ambient conditions. This will provide direct evidence for how the ambient imaging conditions influence the PFM resolution. To determine the resolution profiles across naturally occurring $180^{\circ}$ domain are fitted using the 25-75 criteria and the resolution from the mixed piezoresponse and the phase only signal are determined. A schematic of the effects investigated in this chapter are shown in Figure 4.2. In UHV, Figure 4.2a, the external parameters that influence the resolution are the tip radius and the permittivity of free space. It would not seem to make much difference measuring in UHV or air since the relative permittivity of air is 1.000589 at standard temperature and pressure. However, water is also present in the atmosphere and will coat the surface of the ferroelectric. As a result a meniscus will form between the tip and surface water layer the size of which is determined by the relative humidity. Water, being a polar molecule has a much higher relative permittivity of $\sim 80$ and can have a great influence on the resolution. Finally Figure 4.2c shows how the 
polarization, piezoresponse and phase response are expected to change across a $180^{\circ}$ domain wall and shows examples of the resolution defined by the 25-75 rule.

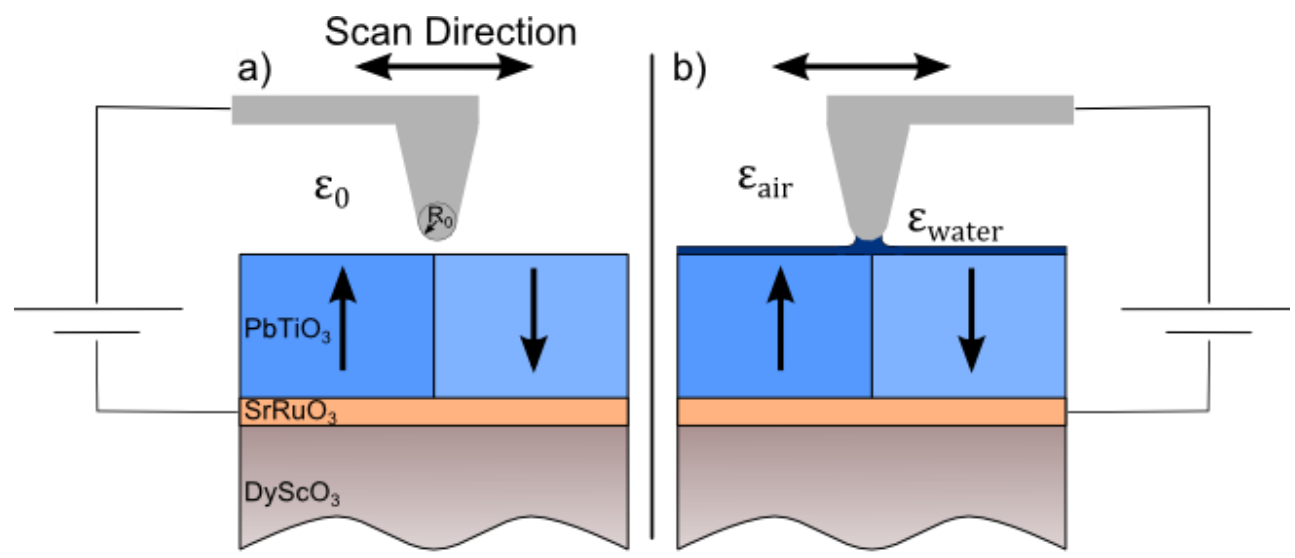

c)

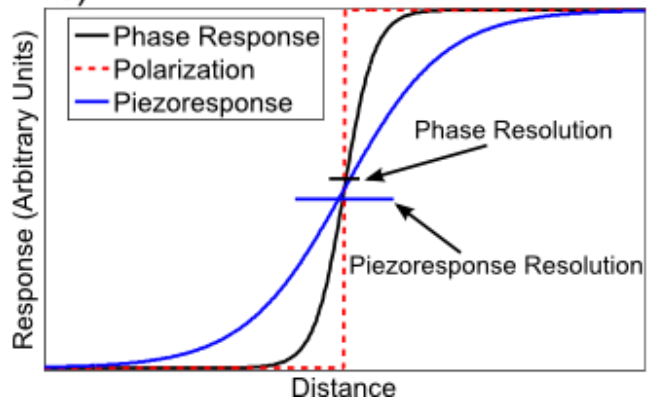

Figure 4.2 Schematic of PFM imaging in a) UHV, b) air and c) relation between the polarization and the piezoresponse and phase signals

\subsection{Experimental}

Naturally occurring $180^{\circ}$ domain walls in a $\mathrm{PbTiO}_{3}$ thin film were used to determine the resolution of the PFM in UHV and in atmospheric conditions. First a $\mathrm{PbTiO}_{3}$ film of 30 $\mathrm{nm}$ was grown by PLD on a $\mathrm{DyScO}_{3}$ substrate with a $5 \mathrm{~nm} \mathrm{SrRuO}_{3}$ bottom electrode for electrical contact for the PFM experiment. After growth the $\mathrm{PbTiO}_{3}$ sample was transferred in UHV into the PFM chamber for characterization. PFM amplitude and phase images were recorded after optimizing the AC voltage and frequency, tip contact force, scan rate and the lock-in amplifier settings. During the PFM measurements the pressure in the chamber was 1011 mbar. After the UHV PFM images were recorded the AFM tip was retracted from the $\mathrm{PbTiO}_{3}$ surface and the chamber was exposed to atmosphere by removing one of the windows. The AFM tip was then brought back into contact with the surface and once again 
PFM amplitude and phase images were recorded after optimizing the measurement parameter settings. The time between subsequent PFM measurements in UHV and atmosphere was approximately 30 minutes which should minimize any time varying effects.

The amplitude and phase images are combined to obtain images of the full piezoresponse with $\operatorname{PR}(\mathrm{x}, \mathrm{y})=\mathrm{A}(\mathrm{x}, \mathrm{y}) \times \cos [\theta(\mathrm{x}, \mathrm{y})]$, where $\mathrm{A}(\mathrm{x}, \mathrm{y})$ is the piezoresponse amplitude image and $\theta(\mathrm{x}, \mathrm{y})$ is the piezoresponse phase image. Then profiles are extracted across the $180^{\circ}$ domain walls along the fast scan axis where the tip is moving perpendicular to the domain wall for the mixed piezoresponse image and the phase image. These profiles are then fit using Boltzmann fit where $\mathrm{PR}(\mathrm{x})=\frac{\mathrm{PR}^{-}+\left(\mathrm{PR}^{+}-\mathrm{PR}^{-}\right)}{1+\mathrm{e}^{\frac{\left(\mathrm{x}-\mathrm{x}_{0}\right)}{\mathrm{x}_{\mathrm{d}}}}}$ where $\mathrm{PR}^{-}$and $\mathrm{PR}^{+}$are the positive and negative constant piezoresponse signal on each side of the domain wall and $\mathrm{x}_{0}$ is the position where the signal changes sign. Using the 25-75 rule the resolution of the domain wall is $\mathrm{w}_{\mathrm{r}}=2 \mathrm{x}_{\mathrm{d}}$. The lock-in amplifier only records values of the phase from $-180^{\circ}$ to $180^{\circ}$ so if the phase signal decreases from $-180^{\circ}$ it will "jump" to $180^{\circ}$. An example of this jump is shown in Figure $4.3 \mathrm{a}$ while the proper evolution of the signal across a $180^{\circ}$ is shown in Figure 4.3b. As a result the domain wall appears to be infinitely sharp and will not be reflective of the true resolution of the instrument. Domain wall profiles that exhibit this "jumping" behavior are therefore excluded from the calculation of the PFM resolution.
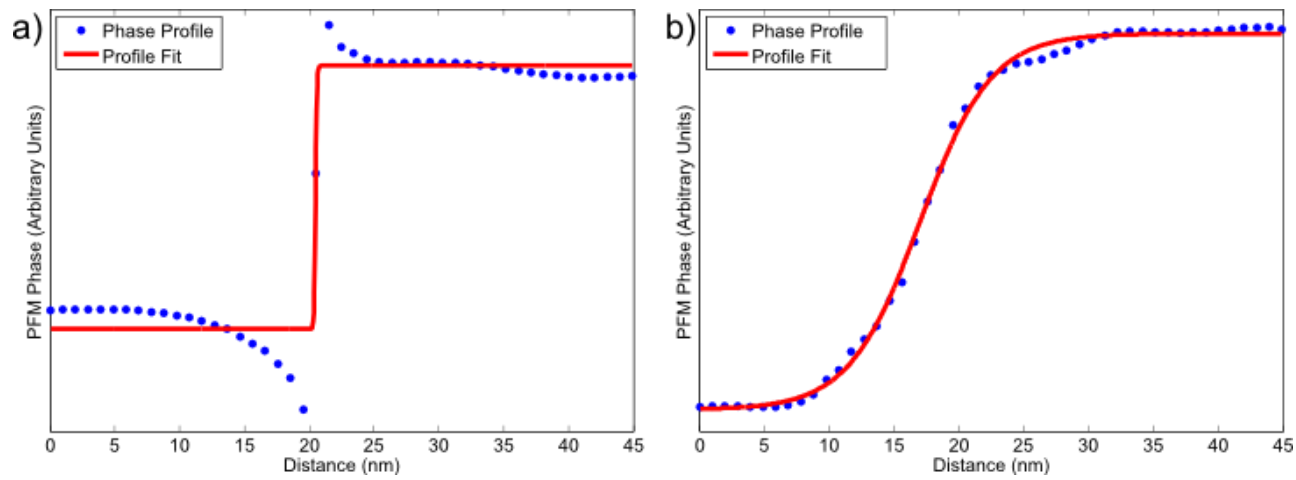

Figure 4.3 Phase profile across a $180^{\circ}$ domain wall showing a) a discontinuous b) a continuous jump in the phase signal.

\subsection{Results}

The mixed piezoresponse and phase PFM images for the UHV and ambient measurements are shown in Figure 4.4. The piezoresponse images are displayed in Figure 4.4a for UHV and Figure 4.4b for the measurement in air. Similarly the phase images for the UHV and air experiments are shown in Figure $4.4 \mathrm{c}$ and $4.4 \mathrm{~d}$ respectively. In both UHV and air $180^{\circ}$ 
domains are observed as indicated by the alternating dark and light contrast regions in the mixed signal and phase response. Additionally $90^{\circ}$ domains are also seen in the piezoresponse images as the out of plane piezoresponse goes to zero where the polarization of the $\mathrm{PbTiO}_{3}$ lies in the plane of the film surface. The domain walls used for the profile fitting are highlighted and shown below the phase image. These domain walls were chosen because they were relatively straight and long providing many profiles for analysis across each domain wall.

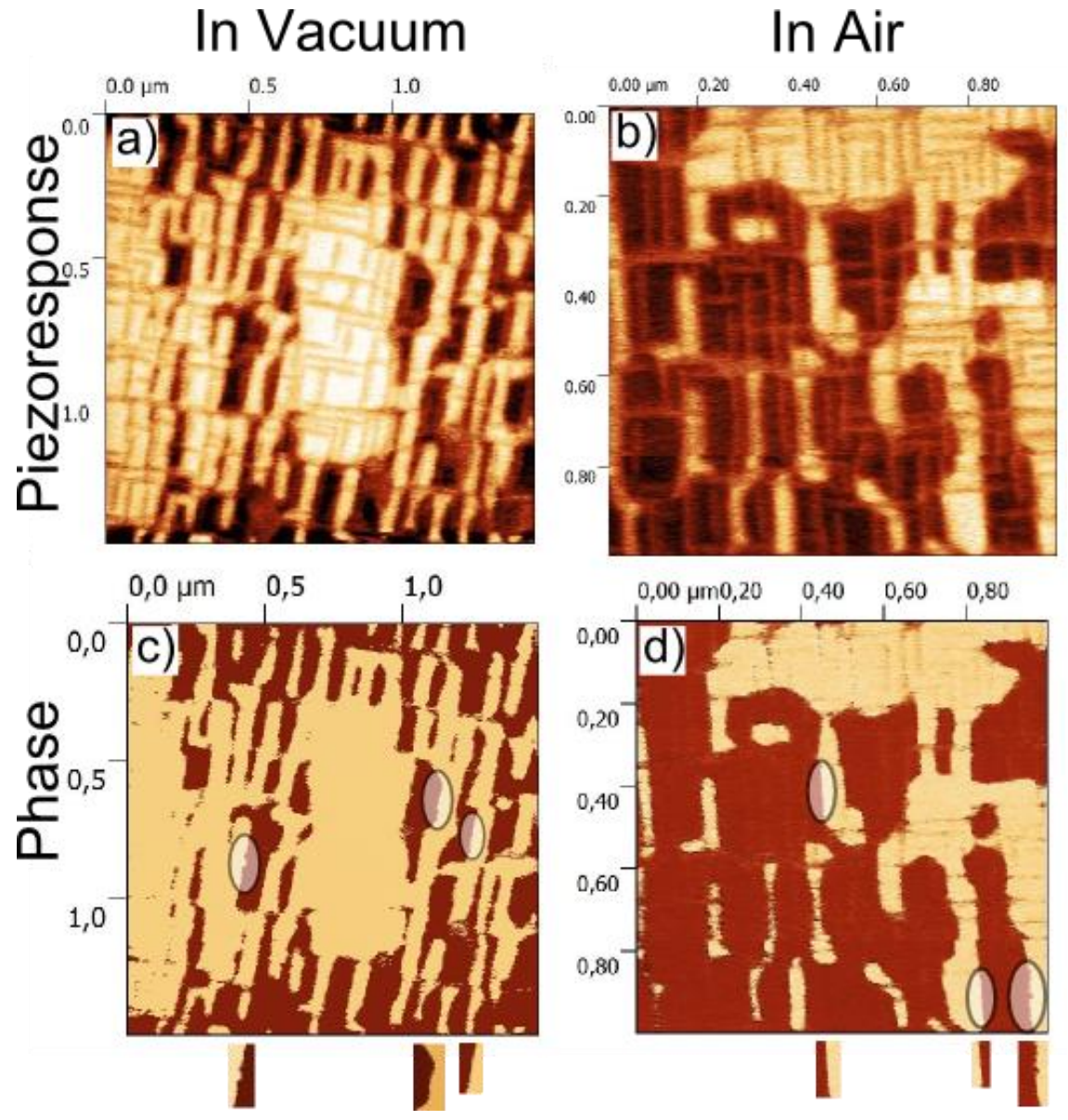

Figure 4.4 Out of plane PFM images from a 30nm $\mathrm{PbTiO}_{3}$ film a) piezoresponse in $\mathrm{UHV}$, b) piezoresponse in air, c) phase in UHV and d) phase in air.

The resulting resolution extracted from the domain wall profiles are shown in Figure 4.5. The UHV and air resolution for the mixed piezoresponse signal are shown in Figure 4.5a. For all of three domain walls analyzed the UHV PFM measurement always showed a better resolution. Combining all three domain walls the average resolution of the mixed 
piezoresponse in UHV was $10.66 \pm 4.06 \mathrm{~nm}$ compared to $16.95 \pm 5.89 \mathrm{~nm}$ when imaging in air. A similar trend is observed for the resolution determined from the phase profiles, Figure $4.5 \mathrm{~b}$, where the UHV resolution for each domain wall is smaller than when measured in air. The phase response resolution for UHV PFM was $1.46 \pm 0.30 \mathrm{~nm}$ compared to $2.72 \pm 1.83$ $\mathrm{nm}$. It is also interesting to note that while the error bars for the piezoresponse resolution are of the same magnitude for the phase resolution the error bars on the UHV measurement are an order of magnitude lower than the air measurement.

a)

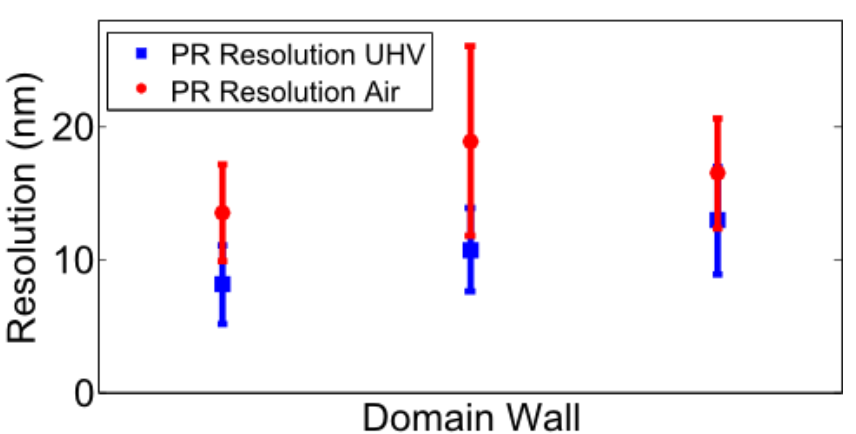

b)

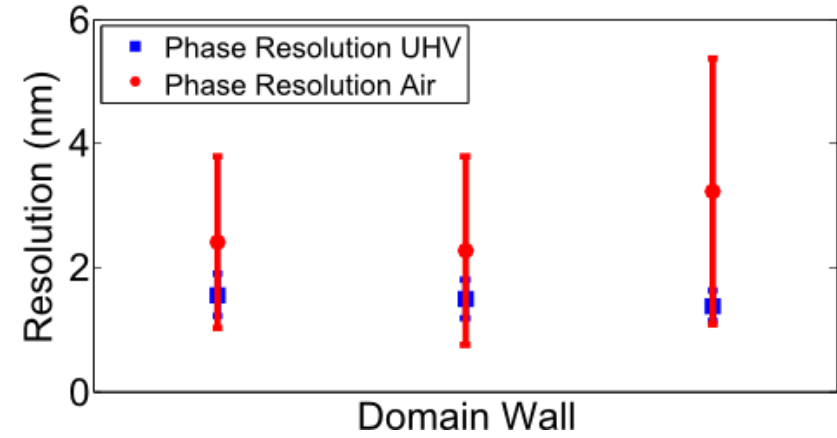

Figure 4.5 Average a) piezoresponse resolution and b) phase resolution for the three domain walls in the UHV and air PFM images

\subsection{Discussion}

The increase in the PFM resolution due to imaging the $\mathrm{PbTiO}_{3}$ film in UHV vs. air was $\sim 1.5$ for the mixed signal piezoresponse and the phase signal. This value does not match up with the analytical model which predicted that the resolution is directly proportional to the relative permittivity of the external medium. In a humid atmosphere we would expect a large difference of about 80 times between the measurements. If however, the air was dry then the difference in the resolution should be negligible. The small improvement in the resolution could be due only a few monolayers of water being present on the film surface preventing a 
meniscus from forming. As a result the influence of the water on the resolution is reduced compared to a scenario where a larger meniscus surrounds a large portion of the tip. Another explanation is that the water layer is not playing any role in the change in resolution observed in the two environments and a change in the tip radius is responsible. Tip damage or wear during the engagement of the tip with the film surface and or while the tip is scanned over the surface during imaging are expected to result in an increase in the tip radius. If the tip was damaged a drastic change in the resolution would be observed mid-image while tip wear would result in a gradually change in the resolution. No sudden change in the resolution was seen so tip damage can be ruled out. Since the imaging in air was performed after the UHV measurement the resulting change in the resolution from a change in tip wear cannot be completely ruled out. However, there were no large surface features so the tip wear should be minimal.

Another result from these two experiments is the large difference in the error bars for the phase resolution. The error bars while imaging in air $\sim 6$ times larger than in UHV. The phase response acts similar to a threshold operation on the mixed signal piezoresponse image. Therefore the phase will change rapidly at the crossover between positive and negative piezoresponse and will be strongly affected by the background noise [7]. Therefore, the error bars in the phase resolution are indicative of the background noise in the image. Since the PFM in air was performed with one of the chamber windows open background sound during the measurement will result in an increased signal noise during the measurement.

\subsection{Conclusion}

Performing PFM in UHV was found to improve the resolution of both the mixed signal piezoresponse and the phase response by a factor of $\sim 1.6$. This change in the resolution is due to either the presence of a surface layer of water when the $\mathrm{PbTiO}_{3}$ is exposed to air or from wear of the tip during the two measurements. Tip wear is expected to be minimal due to the smooth film surface. In order to fully understand the influence of the surface water layer and formation of a tip -surface meniscus PFM images in a controlled humidity environment should be performed. Finally, background sound during the measurement in air resulted in an increase in the background noise in the PFM in air images creating a large error in the phase resolution of the domain walls. 


\section{References}

[1] C. S. Ganpule, V. Nagarajan, H. Li, A. S. Ogale, D. E. Steinhauer, S. Aggarwal, et al., "Role of 90 degrees domains in lead zirconate titanate thin films," Applied Physics Letters, vol. 77, pp. 292-294, Jul 102000.

[2] V. V. Shvartsman, W. Kleemann, R. Haumont, and J. Kreisel, "Large bulk polarization and regular domain structure in ceramic $\mathrm{BiFeO}_{3}$," Applied Physics Letters, vol. 90, pp. -, 2007.

[3] C. Halperin, S. Mutchnik, A. Agronin, M. Molotskii, P. Urenski, M. Salai, et al., "Piezoelectric Effect in Human Bones Studied in Nanometer Scale," Nano Letters, vol. 4, pp. 1253-1256, 2004/07/01 2004.

[4] S. V. Kalinin, B. J. Rodriguez, S. Jesse, T. Thundat, and A. Gruverman, "Electromechanical imaging of biological systems with sub-10nm resolution," Applied Physics Letters, vol. 87, pp. -, 2005.

[5] Rayleigh, "XV. On the theory of optical images, with special reference to the microscope," Philosophical Magazine Series 5, vol. 42, pp. 167-195, 1896/08/01 1896.

[6] A. J. den Dekker and A. van den Bos, "Resolution: a survey," Journal of the Optical Society of America A, vol. 14, pp. 547-557, 1997/03/01 1997.

[7] S. V. Kalinin, S. Jesse, B. J. Rodriguez, J. Shin, A. P. Baddorf, H. N. Lee, et al., "Spatial resolution, information limit, and contrast transfer in piezoresponse force microscopy," Nanotechnology, vol. 17, p. 3400, 2006.

[8] A. N. Morozovska, E. A. Eliseev, S. L. Bravina, and S. V. Kalinin, "Resolutionfunction theory in piezoresponse force microscopy: Wall imaging, spectroscopy, and lateral resolution," Physical Review B, vol. 75, p. 174109, 05/17/ 2007. 


\section{Chapter 5}

\section{$\mathrm{PbTiO}_{3}$ and $\mathrm{BiFeO}_{3}$ on $\mathrm{SrRuO}_{3} \mathrm{Nanowires}$}

\subsection{Introduction}

Ferroelectric materials have a found a wide range of applications utilizing the many attractive properties of these materials. Nonvolatile memories, piezoelectric actuators and sensors, high $\mathrm{K}$ dielectrics for capacitors, and tunable microwave devices [1-9] all benefit from the various properties that ferroelectrics offer. In order to optimize the material performance for each application it is necessary to be able to precisely control the crystal structure, defects, and domain configuration in the ferroelectric. Traditionally, altering the material properties was accomplished by doping the ferroelectric with various elements. However, development of thin film growth techniques driven by the promise of new thin film device applications created possibilities to control the properties of a ferroelectric thin film by carefully controlling the boundary conditions under which the film is subjected.

The nature of ferroelectricity, where spontaneous electric polarization is the result of a structural deformation of the unit cell, makes these materials sensitive to the mechanical and electrical environment in which the material is placed. Thin film growth of ferroelectrics significantly expands the range of boundary conditions which a ferroelectric can be subjected. First thin film growth allows for well-defined interfaces between the metallic electrodes and the ferroelectric resulting improved screening of bound surface charges. Secondly, strain engineering is possible utilizing epitaxial growth of ferroelectrics on substrates with different unit cell sizes allowing for precise control of the strain in the ferroelectric and permits the material to be subjected to large strains that would crack bulk materials. These new possibilities spurred many theoretical and experimental studies examining the how the properties of ferroelectric thin films can be altered by precise control of the boundary conditions. 
There have been many theoretical studies analyzing the effect of strain and temperature on the domain structure of ferroelectric films. For $\mathrm{PbTiO}_{3}$, strain-phase diagrams have been calculating using various methods; from simple thermodynamic calculations assuming a single domain state [10], to allowing two domain states [11], all the way to full three dimensional phase field simulations [12]. Qualitatively, these strain-phase diagrams predict a cphase oriented ferroelectric with compressive strain, large tensile strains will result in fully inplane oriented films, and in between alternating c/a polydomains will form. Quantitatively, however, the different theoretical methods and assumptions predict these phases forming at different strains and temperatures.

Experimentally $\mathrm{PbTiO}_{3}$ films have been grown on many different substrates each with different lattice parameters from $\mathrm{SrTiO}_{3}$ to $\mathrm{DyScO}_{3}$ to $\mathrm{MgO}[13,14]$. Through X-ray diffraction studies the $\mathrm{c}$ and $a$ domain abundance were found to depend on the strain imparted on the ferroelectric by the substrate. Microscopic studies using transmission electron microscopy of $\mathrm{PbTiO}_{3}$ grown on a $\mathrm{DyScO}_{3}$ substrate show the a domains are uniformly sized and spaced to minimize misfit strain while remaining laterally coherent with the rest of the film [15].

In all of the studies cited above the entire ferroelectric film was subjected to a uniform set of boundary conditions. Much less is known about how a ferroelectric film behaves when the film is subjected to boundary conditions that periodically change across the lateral dimensions of the film. Changing the boundary conditions across a ferroelectric film will allow new customized domain structures to be fabricated further optimizing the properties of the ferroelectric. One obvious way to subject a continuous film to changing boundary conditions is to pattern the bottom electrode. This has been tried using PLD stencil deposited nanodots of $\mathrm{SrRuO}_{3}$ and $\mathrm{La}_{0.3} \mathrm{Sr}_{0.7} \mathrm{MnO}_{3}$ on $\mathrm{Nb}$ doped $\mathrm{SrTiO}_{3}$ and growing a $\mathrm{PbTiO}_{3}$ film on over the patterned bottom electrode [16]. In this study the entire $\mathrm{PbTiO}_{3}$ film on the $\mathrm{SrRuO}_{3}$ nanodots was in a uniform polarization state and the ferroelectric domains and piezoresponse were not influenced by the presence or absence of a nanodot under a particular region of the $\mathrm{PbTiO}_{3}$ film. For the $\mathrm{PbTiO}_{3}$ on $\mathrm{La}_{0.3} \mathrm{Sr}_{0.7} \mathrm{MnO}_{3}$ nanodots $180^{\circ}$ domains formed in circular patterns around the dots. This pattern was attributed to a metal to insulator transition that occurred as the $\mathrm{La}_{0.3} \mathrm{Sr}_{0.7} \mathrm{MnO}_{3}$ gradually thinned out at the edges of the nanodots. In this study no change in the mechanical boundary conditions took place as the aspect ratio of the patterned dots was small creating a gentle slope up to the maximum height of the dots.

In this chapter we will investigate the influence varying boundary conditions has on the domain structure of a ferroelectric thin film using patterned bottom electrodes. Unlike the previous study, where the only the electrical boundary conditions where changed in a very small region around the nanodots, we will use a self-assembly technique to produce nanowires and nanoislands of $\mathrm{SrRuO}_{3}$ on a $\mathrm{DyScO}_{3}$ substrate. A schematic highlighting the important differences in the boundary conditions between a ferroelectric film on a flat bottom electrode 
and on a self-assembled nanostructured $\mathrm{SrRuO}_{3}$ bottom electrode is shown in Figure 5.1. On a flat bottom electrode, shown in Figure 5.1a, the ferroelectric film is subjected to uniform screening from the bottom electrode and uniform strain in the ferroelectric due to misfit strain. As a result the domain structure in the ferroelectric will have the same pattern over the entire area of the film. For a ferroelectric film on a patterned bottom electrode, Figure 5.1b, the boundary conditions change depending on the location of the ferroelectric film is with respect to the underlying nanostructure. The difference between the conducting $\mathrm{SrRuO}_{3}$ and insulating $\mathrm{DyScO}_{3}$ should produce a large change in the electrical boundary conditions as screening charges will only be present when a ferroelectric is grown on the $\mathrm{SrRuO}_{3}$. Furthermore, the self-assembly method employed is capable of producing high aspect ratio patterns creating sharp edges which the ferroelectric thin film must conform, allowing the possibility of strain at the edges of the wire patterns therefore changing the mechanical boundary condition across the ferroelectric film.

\section{a)}

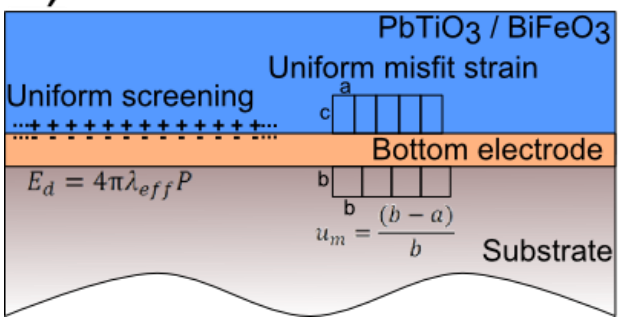

b)

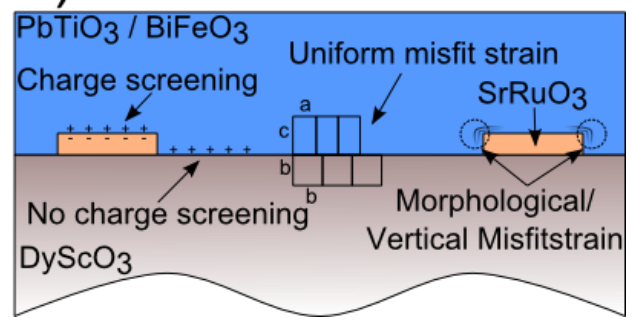

Figure 5.1 Schematic boundary conditions acting on $\mathrm{PbTiO}_{3}$ and $\mathrm{BiFe}_{3}$ films on a) a continuous bottom electrode and b) on a patterned bottom electrode.

\subsection{Experimental}

Thin films of $\mathrm{PbTiO}_{3}$ and $\mathrm{BiFeO}_{3} 25 \mathrm{~nm}$ thick were grown on $5 \mathrm{~nm} \mathrm{SrRuO}_{3}$ nanowires to investigate influence of varying boundary conditions on the domain structure of a ferroelectric thin film. A self-assembly technique using pulsed laser deposition of $\mathrm{SrRuO}_{3}$ on mixed terminated $\mathrm{DyScO}_{3}$ substrates was used to pattern the bottom electrode [17]. Depending on the initial pattern of the mixed termination different $\mathrm{SrRuO}_{3}$ nanostructures are obtained. If the mixed termination is randomly arranged on the substrate then $\mathrm{SrRuO}_{3}$ nanoislands are formed. In some cases the mixed termination is aligned with the vicinal steps of the $\mathrm{DyScO}_{3}$ and when this happens nanowires of $\mathrm{SrRuO}_{3}$ are obtained. The nanowires run across the surface and can be electrically contacted at the edges of the sample.

$\mathrm{BiFeO}_{3}$ and $\mathrm{PbTiO}_{3}$ films were grown on $\mathrm{SrRuO}_{3}$ nanowires to investigate the influence periodically modulated boundary conditions has on the domain structure. Using 
these samples the electrical and mechanical boundary conditions change periodically across the sample perpendicular to the underlying nanowires. Parallel to the nanowires each region of the ferroelectric film (on or off the nanowires) is subjected to the same boundary conditions. To ensure high quality epitaxial growth and to minimize contamination from the atmosphere the nanowires and ferroelectric film are grown in a single deposition run. Afterwards, the domain structures in the ferroelectric films are investigated using PFM. This technique allows the local polarization orientation of the ferroelectric films to be mapped on the nanoscale allowing a direct correlation between the domain structure of the film and the position relative to the nanowires. Further investigation to determine exactly how and where the nanowires are influencing the $\mathrm{PbTiO}_{3}$ domain structure is done using TEM. Using TEM the positions of the atomic columns are mapped allowing structural a/c domains to be identified. Since TEM requires a cross section of the samples the locations of the $\mathrm{a} / \mathrm{c}$ domains through the thickness of the film are mapped and their locations relative to the nanowires are easily determined.

To understand how the thin film growth process influences the boundary conditions and hence the final domain structure a series of $3 \mathrm{~nm} \mathrm{PbTiO}_{3}$ and $\mathrm{BiFeO}_{3}$ thin films were grown on $5 \mathrm{~nm} \mathrm{SrRuO}$ islands. During these experiments $3 \mathrm{~nm}$ films were grown on the nanoislands and then the surface of the film was characterized using AFM. Afterwards another $3 \mathrm{~nm}$ of material was deposited and then characterized again. In this way the evolution in the morphology of the $\mathrm{PbTiO}_{3}$ and $\mathrm{BiFeO}_{3}$ films during the initial few nanometers of film growth was explored. To insure that growth and morphology of the films is not influenced by contamination of water or other molecules present in the atmosphere the films were kept in UHV conditions during the entire growth and characterization process utilizing an in situ AFM for the characterization steps. A schematic of the experimental process of the ferroelectric film growth on nanowires and nanoislands is shown in Figure 5.2. 


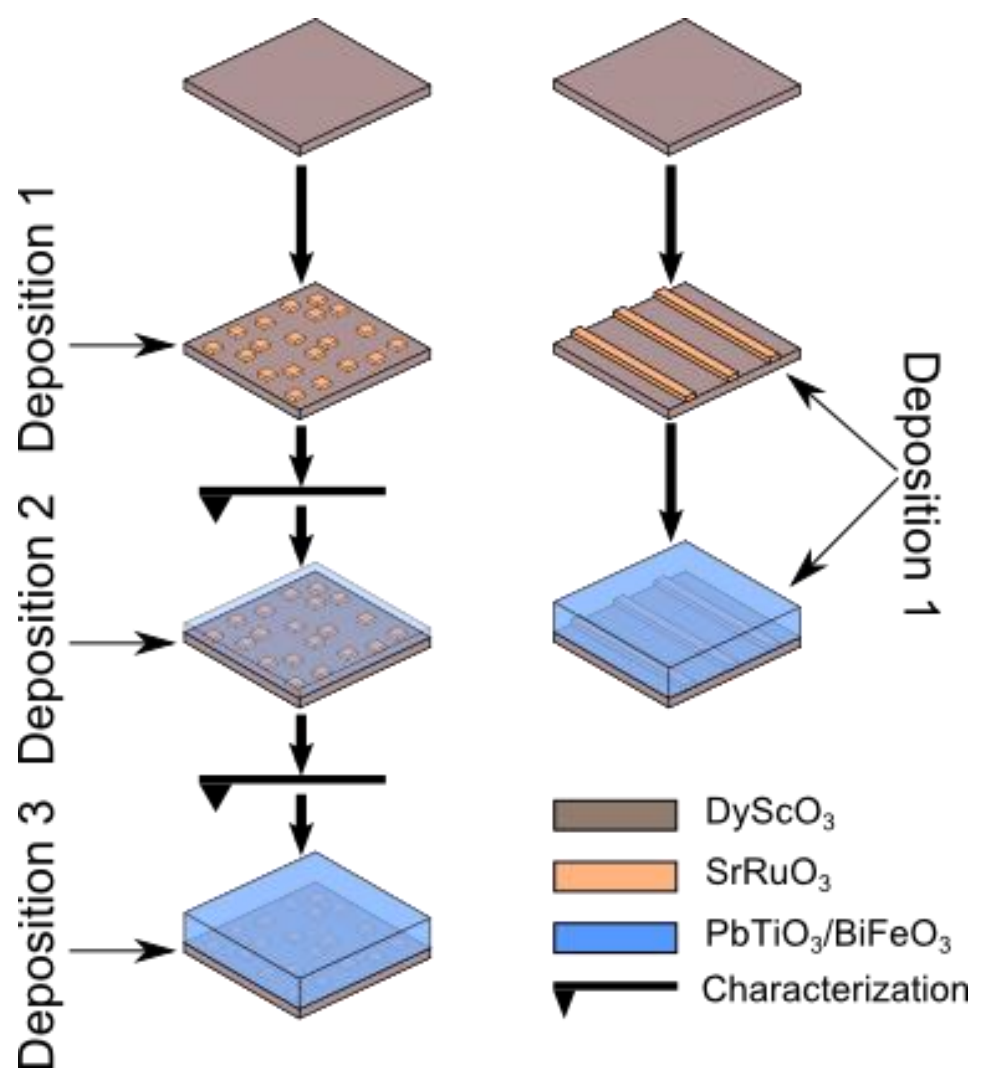

Figure 5.2 Schematic of the deposition procedure used for the samples in this chapter. Left) schematic of the growth on $\mathrm{SrR} u \mathrm{O} 3$ nanoislands study, $5 \mathrm{~nm}$ high $\mathrm{SrR} u \mathrm{O} 3$ nanoislands were deposited and then characterized in situ using AFM. Next $3 \mathrm{~nm}$ of ferroelectric was deposited and then characterized using AFM/PFM before an additional $3 \mathrm{~nm}$ of ferroelectric was grown and then characterized for a final time. Right) schematic of the ferroelectric on $\mathrm{SrR} u \mathrm{O} 3$ nanowires study, the $\mathrm{SrR} u \mathrm{O} 3$ nanowires and ferroelectric film were grown in a single deposition run.

\subsection{Results}

\subsubsection{Growth of $\mathrm{PbTiO}_{3}$ and $\mathrm{BiFeO}_{3}$ on $\mathrm{SrRuO}_{3}$ nanowires}

Atomic force microscopy images of the mixed terminated $\mathrm{DyScO}_{3}$ substrates used for the nanowire growth of $\mathrm{SrRuO}_{3}$ are shown in Figure 5.3. In Figure 5.3a, the substrate used for $\mathrm{PbTiO}_{3}$, the mixed termination is seen in the surface topography with the appearance of $2 \AA$ steps corresponding to half a unit cell. For the substrate used for the $\mathrm{BiFeO}_{3}$, Figure 5.3c, the mixed termination is not easily seen in the surface topography. However, for both 
substrates the presence of mixed termination is clearly observed in the phase contrast of a tapping mode AFM image, Figures $5.3 \mathrm{~b}$ and $\mathrm{d}$.
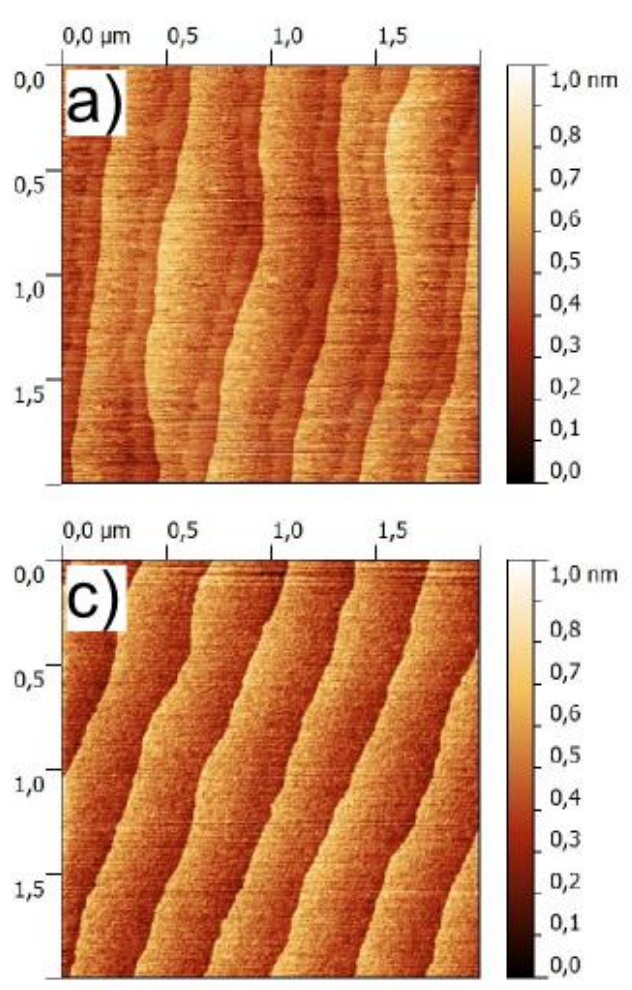
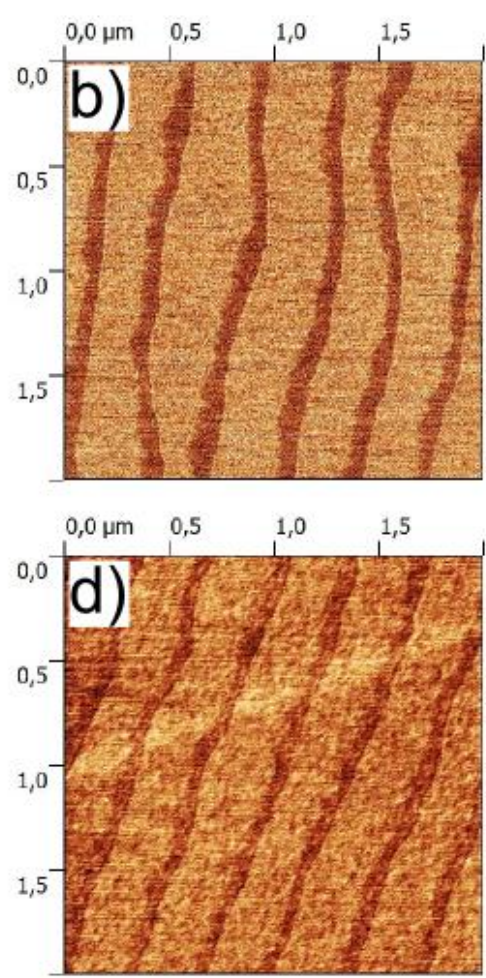

Figure 5.3 a) surface topography and b) phase contrast of mixed terminated $\mathrm{Dy} \mathrm{ScO}_{3}$ substrate used for the growth of $\mathrm{PbTiO}_{3}$ on nanowires; c) surface topography and d) phase contrast of mixed terminated $\mathrm{DySCO}_{3}$ substrate used for the growth of $\mathrm{BiFeO}_{3}$ on nanowires

The two different chemical surfaces, $\mathrm{SrO}_{2}$ and $\mathrm{DyO}$, will interact slightly differently with the AFM tip producing different phase shifts in the response of the cantilever providing the contrast observed in the phase images. In these substrates the mixed termination aligns along the vicinal surface steps. Since the surface steps run across the entire substrate the resulting nanowires will also run across the length of the substrate where they can be contacted at the edges of the substrate. The periodicity of the surface steps provides the periodicity of the mixed termination, and after $\mathrm{SrRuO}_{3}$ growth, the nanowire periodicity. The surface step periodicity is determined from a 2D fast Fourier transform analysis of the surface topography AFM image. For the substrate used for the growth of $\mathrm{PbTiO}_{3}$, Figure 5.3a and $\mathrm{b}$, this periodicity is $\sim 400 \mathrm{~nm}$ while for the substrate where the $\mathrm{BiFeO}_{3}$ will be grown, Figure $5.3 \mathrm{c}$ and $\mathrm{d}$, the surface periodicity is $\sim 325 \mathrm{~nm}$. What cannot be determined from AFM is which surface 
belongs to which termination. Although the periodicity of the wires will be the same one case will result in wide wires with skinny spacing between them and vice versa.

Surface topography images taken by AFM after the growth of $5 \mathrm{~nm}$ SrRuO3 nanowires and $25 \mathrm{~nm}$ of $\mathrm{PbTiO} 3 / \mathrm{BiFeO} 3$ are shown in Figure 5.4. Although thickness of the bottom electrode and ferroelectric film are the same for each ferroelectric material there is a striking difference in the surface topography. For the PbTiO3, Figure 5.4a, the underlying nanowires are clearly visible in the surface topography showing the $\mathrm{SrRuO} 3$ growth resulted in skinny continuous nanowires. The film covers the surface creating a conformal coating maintaining the topography of the nanowires. However, there is some flattening of the surface, originally $5 \mathrm{~nm}$ high, the surface height difference is now only $2 \mathrm{~nm}$ after the growth of $25 \mathrm{~nm}$ of PbTiO3. On the other hand, for the BiFeO3, Figure 5.4b, the top surface is atomically smooth with only unit cell high steps on the surface. There is no visible evidence of the SrRuO3 nanowires in the surface topography. This could be an indication that the $\mathrm{SrRuO} 3$ did not form nanowires and the growth resulted in a flat bottom electrode. However, conductivity measurements, which will be discussed in Chapter 5 particularly Figure $5.2 \mathrm{~b}$ and $\mathrm{d}$, confirm the presence of $\mathrm{SrRuO} 3$ nanowires underneath the flat $\mathrm{BiFeO} 3$ film.
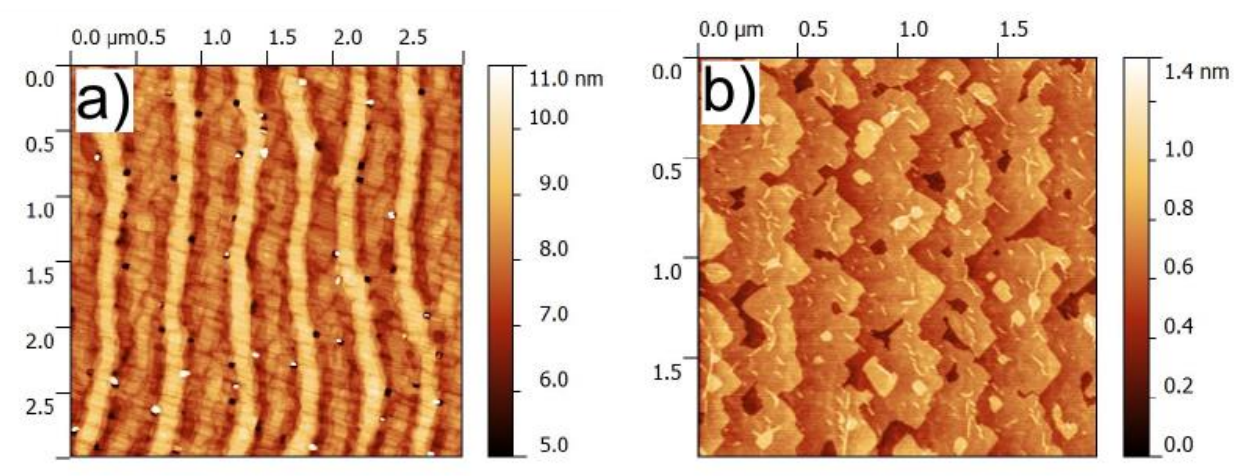

Figure 5.4 AFM surface topography of $25 \mathrm{~nm}$ a) $\mathrm{PbTiO}_{3}$ and b) $\mathrm{BiFe}_{3}$ on $5 \mathrm{~nm} \mathrm{SrR}_{2} \mathrm{O}_{3}$ nanowires

\subsubsection{Domain structure of $\mathrm{PbTiO}_{3}$ and $\mathrm{BiFeO}_{3}$ on $\mathrm{SrRuO}_{3}$ nanowires}

To investigate the domain structure of the PbTiO3 and $\mathrm{BiFeO} 3$ films PFM was performed and the resulting images are shown in Figures 5.5 and 5.6 for PbTiO3 and $\mathrm{BiFeO} 3$ respectively. The surface topography of the PbTiO3 during the PFM measurements is shown in Figure 5.5a. 

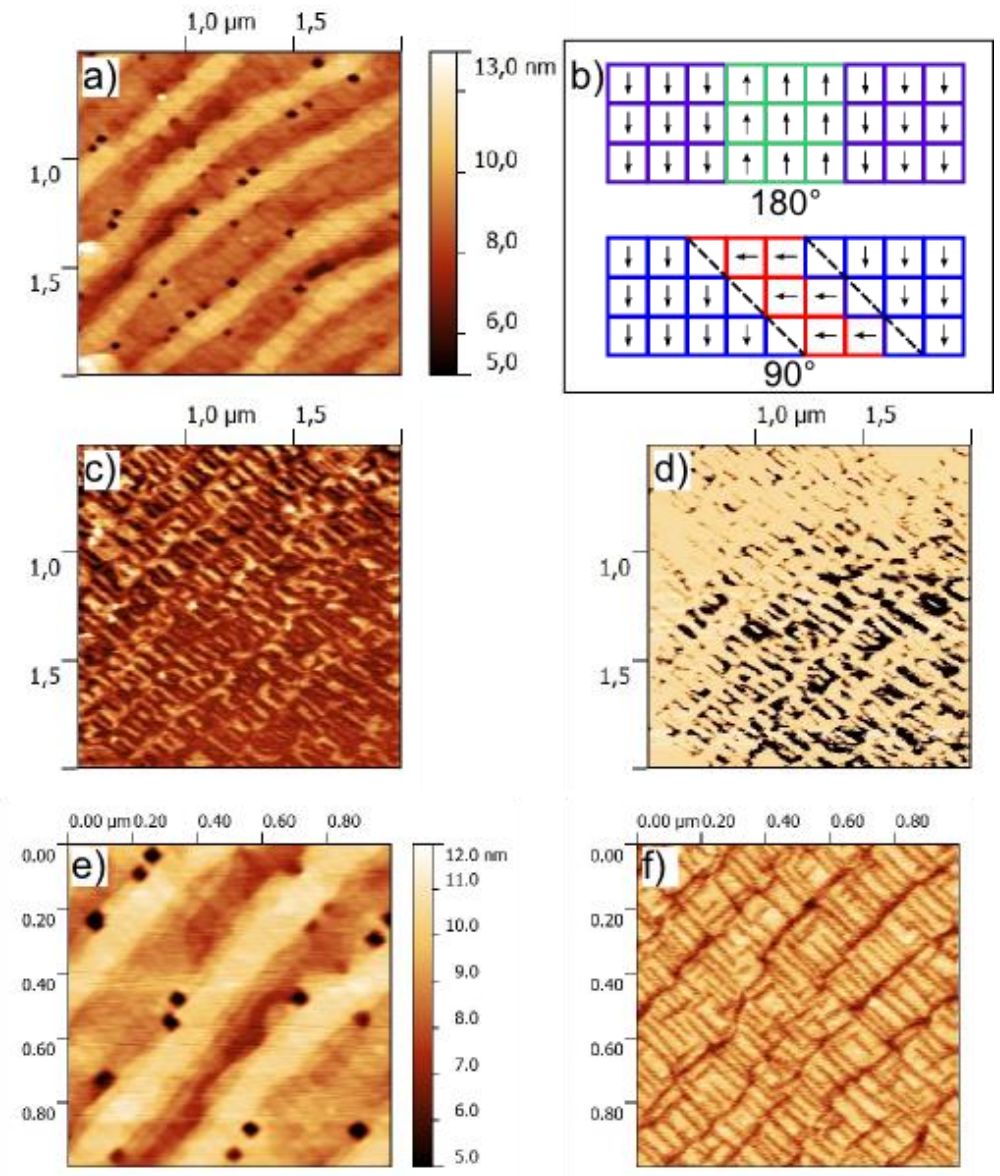

Figure 5.5 a) Surface topography of $\mathrm{PbTiO}_{3}$ on $\mathrm{SrRu} \mathrm{O}_{3}$ nanowires b) schematic of $180^{\circ}$ and $90^{\circ}$ domains c) corresponding out of plane amplitude and d) phase images for the $\mathrm{PbTiO}_{3}$ film. e) Surface topography and f) out of plane amplitude image after switching to show the $90^{\circ}$ domain structure.

For the $\mathrm{PbTiO} 3$ two types of domain structures are observed; $180^{\circ}$ and $90^{\circ}$, these domain structures are shown schematically in Figure 5.5b. As is seen in the out of plane PFM amplitude and phase images, Figures $5.5 \mathrm{c}$ and d respectively, the $180^{\circ}$ domains align across the nanowires, starting and stopping at the edges of the nanowire. As a result, perpendicular to the nanowires the $180^{\circ}$ domain periodicity adopts the periodicity of the nanowires. In the direction of the nanowires the $180^{\circ}$ domains have a characteristic striped domain pattern. In this direction is no significant difference between the periodicity of the $180^{\circ}$ domains on or off the nanowires. In the initial out of plane amplitude image, Figure $5.5 \mathrm{c}$, a few $90^{\circ}$ domains are observed. However, due to the much larger contrast of the $180^{\circ}$ domains the $90^{\circ}$ are hard to visualize. Therefore a region of the film was switched using a DC voltage applied to the AFM 
tip so the film is in a single out of plane domain state. After switching the $90^{\circ}$ domains are clearly visible in the out-of plane amplitude image as regions where the out of plane PFM amplitude becomes zero. The topography of the surface during the $90^{\circ}$ domain investigation is shown in Figure 5.5d and the PFM image of the $90^{\circ}$ domains are shown in Figure 5.5e. The $90^{\circ}$ domains also reflect the underlying nanowire periodicity but in a different way from the $180^{\circ}$ domains. There is a clear preference for the $90^{\circ}$ domains to form along the edges of the nanowires. In the rest of the film there $90^{\circ}$ domains prefer to align perpendicular to the nanowires although there are some small domains parallel to the nanowires.
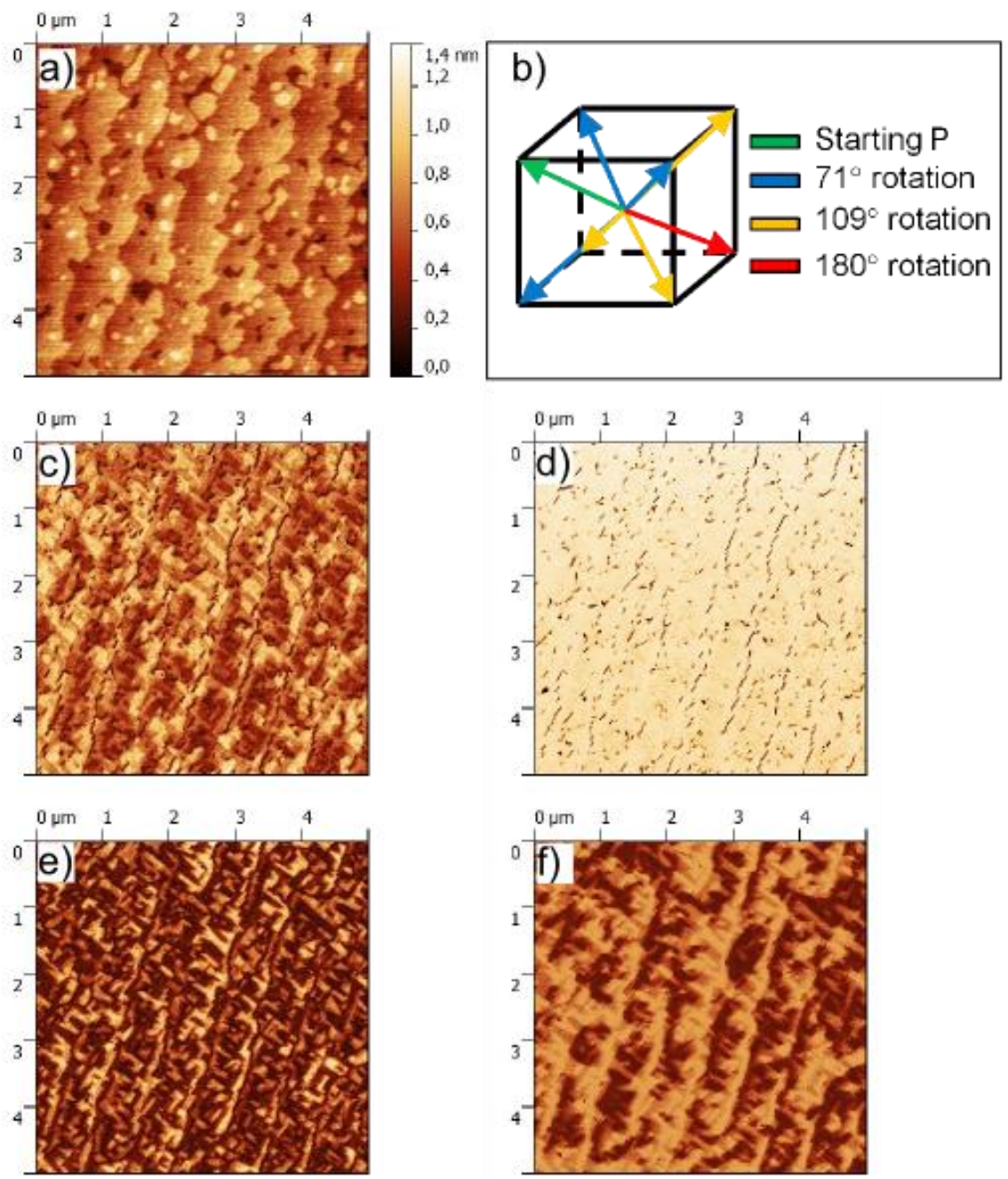

Figure 5.6 a) Surface topography of $\mathrm{BiFeO}_{3}$ on $\mathrm{SrR}_{\mathrm{R}} \mathrm{O}_{3}$ nanowires b) schematic of domain structures in $\mathrm{BiFe}_{3}$ c) corresponding out of plane amplitude and d) phase and in-plane e) amplitude and f) phase images. 
The surface topography and corresponding out of plane and in-plane PFM images for the $\mathrm{BiFeO} 3$ film are shown in Figure 5.6. The surface topography shows periodic unit cell steps with the same periodicity of the mixed termination of the $\mathrm{DyScO} 3$ substrate used for the $\mathrm{SrRuO} 3$ nanowire growth. The majority of out of plane polarization, Figure 5.6e and $\mathrm{f}$, for the $\mathrm{BiFeO} 3$ film is oriented down, towards the substrate which is typical for $\mathrm{BiFeO} 3$ grown on flat $\mathrm{SrRuO} 3$ bottom electrode [18, 19] although there are some small regions of upward oriented polarization. For the in-plane polarization all four of the possible domain variants are present in the film, Figure 5.6e and f. Combining the out of plane and in-plane polarization information the $\mathrm{BiFeO} 3$ film contains only $71^{\circ}$ domain walls. There is no difference in the types of the domains on or off the nanowires. There is a slight alignment of out of plane polarization along the nanowires. For the in-plane polarization there is once again there is some alignment with the underlying nanowires. Besides these few domains the rest of the inplane domain walls do not align with the nanowires. Instead of lying at $0^{\circ}$ and $90^{\circ}$ to the nanowires the in-plane domain walls are at $\sim 21^{\circ}$ and $\sim 67^{\circ}$ to the nanowire direction.

To investigate the microscopic origins for the preferential alignment of the $90^{\circ}$ domains transmission electron microscopy was performed on the $\mathrm{PbTiO}_{3}$ film looking at slices perpendicular and parallel to the nanowires, shown in Figure 5.7.
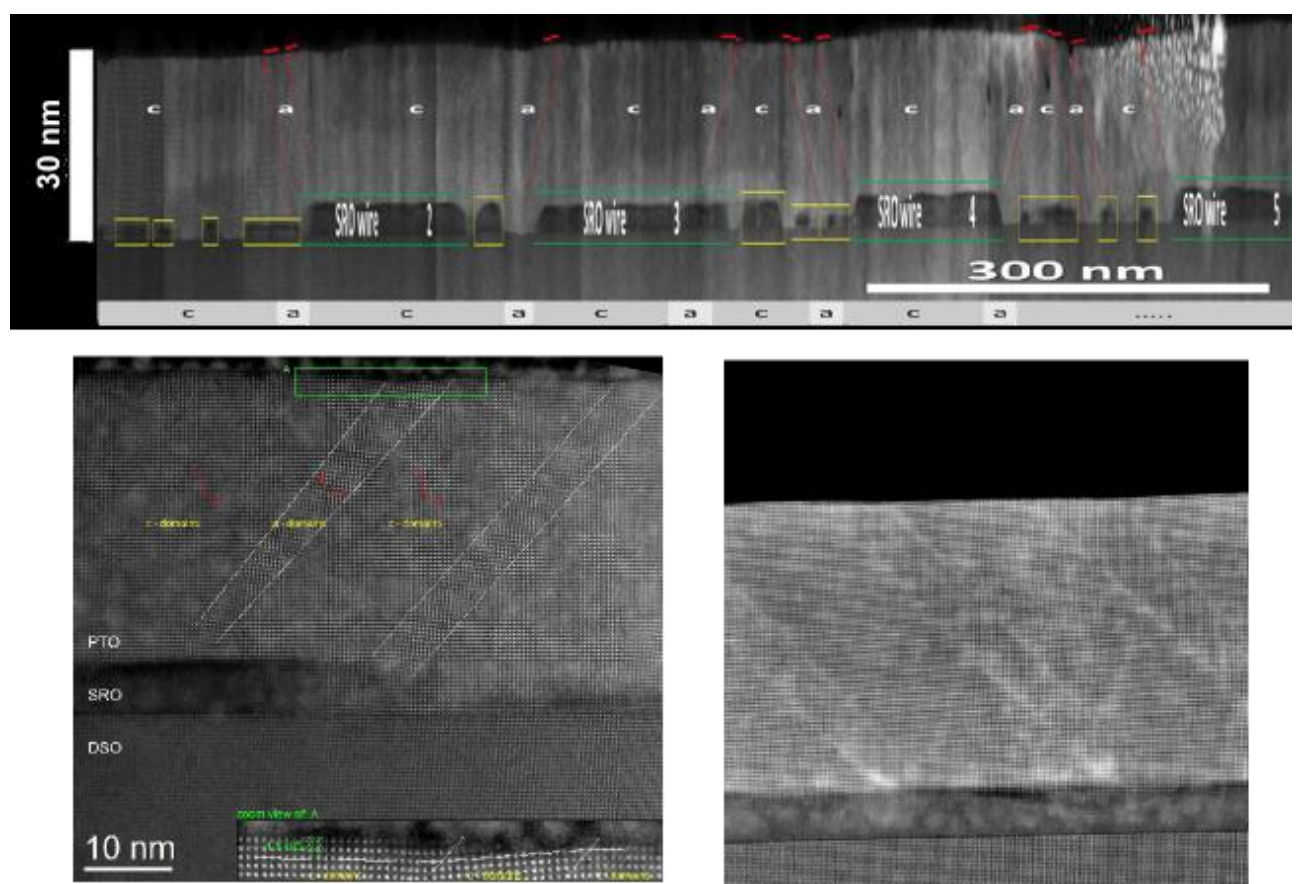

Figure 5.7 a) Composite TEM image of $\mathrm{PbTiO}_{3}$ film on $\mathrm{SrRuO}$ nanowires taken perpendicular to the nanowires note the two axes do not have the same scale, b) zoomed in view of at the edge of a $\mathrm{SrRuO}$ n nanowire. c) TEM parallel and on top of a $\mathrm{SrRuO}$ n nanowire. 
A large composite image showing a cross-section through four $\mathrm{SrRuO}_{3}$ nanowires is shown in Figure 5.7a. The a domains, highlighted by the red lines, are seen originating from either the edges of $\mathrm{SrRuO}_{3}$ nanowires or from small $\mathrm{SrRuO}_{3}$ islands that formed in between the wires and rarely from the either the substrate interface or the flat $\mathrm{SrRuO}_{3}$ interface. A zoomed in image of one nanowire edges, Figure 5.7b, shows two a domains originating with-in close proximity of the $\mathrm{SrRuO}_{3}$ corner. Analyzing the starting locations of 131 a domains $54 \%$ originated from the nanowire edges while $27 \%$ are due to $\mathrm{SrRuO}_{3}$ nanoislands. Finally, a domains from the flat $\mathrm{SrRuO}_{3}$ or the substrate accounted for only $15 \%$ and $3 \%$ of all those $a$ domains observed respectively. The cross-section taken along a $\mathrm{SrRuO}_{3}$ nanowire is shown in Figure 5.7c. In this direction the a domains are evenly spaced showing a nice periodic structure typical for $\mathrm{PbTiO}_{3}$ grown on a flat $\mathrm{SrRuO}_{3}$ bottom electrode [15].

\subsubsection{Growth morphology of $\mathrm{PbTiO}_{3}$ and $\mathrm{BiFeO}_{3}$ on $\mathrm{SrRuO}_{3}$ islands}

The most striking difference between the $\mathrm{PbTiO}_{3}$ and $\mathrm{BiFeO}_{3}$ films on $\mathrm{SrRuO}_{3}$ nanowires is the surface morphology of the two films. To investigation how these two ferroelectrics grow on 3D patterned surfaces and what influence this might have on the domain structure of the ferroelectric $5 \mathrm{~nm}$ high $\mathrm{SrRuO}_{3}$ islands were grown on randomly ordered mixed terminated $\mathrm{DyScO}_{3}$ surface. The starting $\mathrm{SrRuO}_{3}$ nanoisland pattern for the $\mathrm{PbTiO}_{3}$ films is shown in Figure 5.8a.

The $\mathrm{SrRuO}_{3}$ islands form a dense random arrangement of uniformly high $5 \mathrm{~nm}$ islands with island sizes $\sim 50 \mathrm{~nm}$ in diameter. The root mean square surface roughness is 2.48 $\mathrm{nm}$ with an average peak to peak height for each horizontal scan line of $11.11 \mathrm{~nm}$. Although the islands are much closer together than the nanowires in the previous section these samples should still provide a good indication of how the ferroelectric will grow around the edges of the wires where the strongest effects on the domain structure were observed.

The AFM topography images taken in UHV after the deposition of $3 \mathrm{~nm}$ and then another $3 \mathrm{~nm}$ of $\mathrm{PbTiO}_{3}$ are shown in Figure $5.8 \mathrm{~b}$ and $\mathrm{c}$ respectively. In both cases the starting $\mathrm{SrRuO}_{3}$ island pattern is still clearly visible. After the final growth of six total nanometers of material some of the islands have grown together but other than that there is not much difference between the three surfaces.

For the $\mathrm{BiFeO}_{3}$ a similar starting $\mathrm{SrRuO}_{3}$ islands pattern was grown, shown in Figure 5.8c. In this case the surface roughness was slightly smaller with an RMS of $2.25 \mathrm{~nm}$ and an average peak to peak height of $7.67 \mathrm{~nm}$. The AFM topography images after the two $3 \mathrm{~nm}$ $\mathrm{BiFeO}_{3}$ depositions are shown in Figures 5.8e and $\mathrm{f}$. After the deposition of only $3 \mathrm{~nm}$ of $\mathrm{BiFeO}_{3}$, Figure 5.8e, the surface is remarkable changed. Only the tops of the $\mathrm{SrRuO}_{3}$ islands remain visible while most of the regions in-between the islands are filled-in with the $\mathrm{BiFeO}_{3}$. 

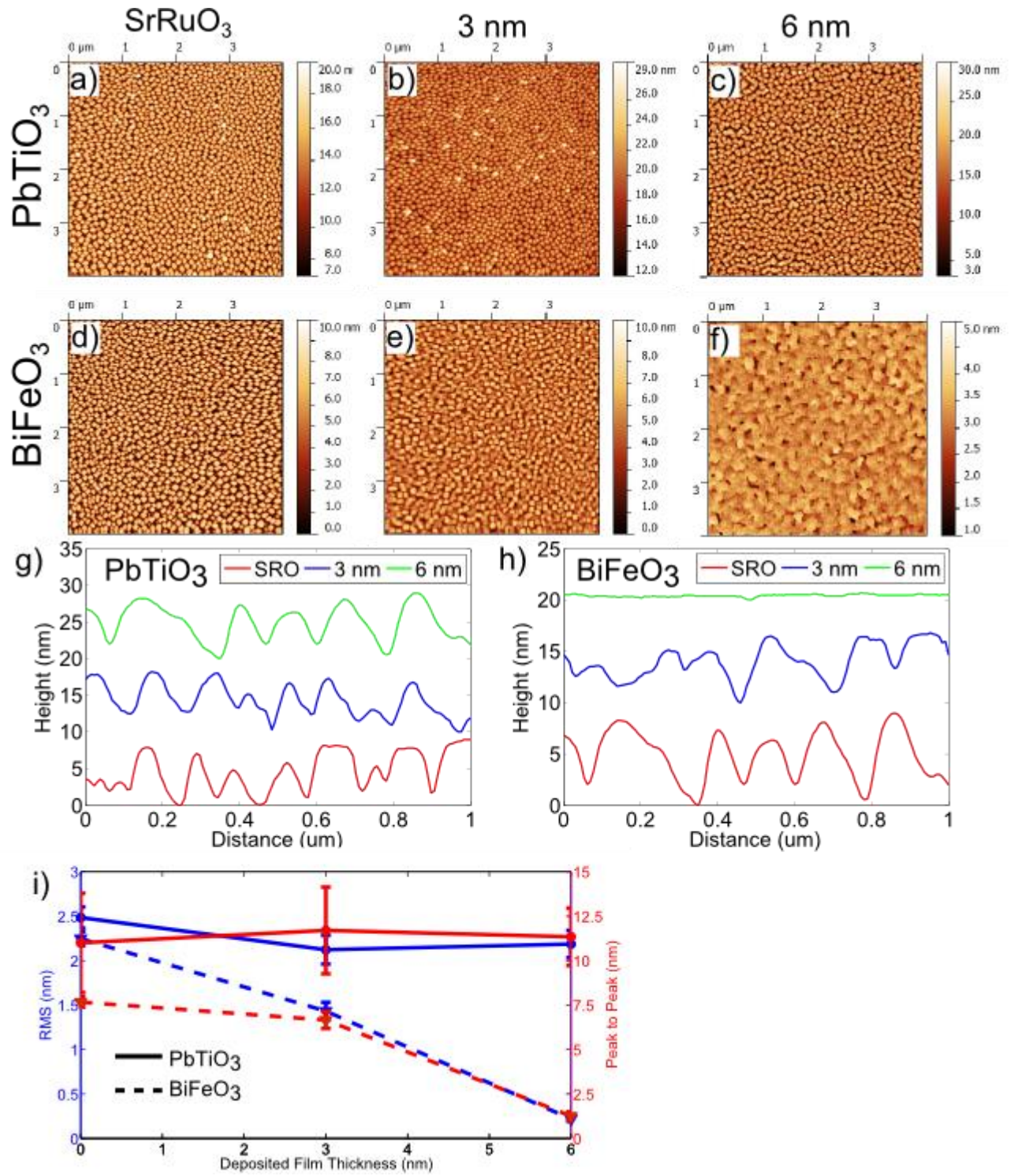

Figure 5.8 a) Surface topography of $5 \mathrm{~nm}$ high $\mathrm{SrRuO}$ nanoislands, b) surface topography after $3 \mathrm{~nm}$ deposition of $\mathrm{PbTiO}_{3}$, c) surface topography after a total of $6 \mathrm{~nm} \mathrm{PbTiO}_{3}$. d) Surface topography of $5 \mathrm{~nm}$ high $\mathrm{SrR} u \mathrm{O}_{3}$ nanoislands, e) surface topography after $3 \mathrm{~nm}$ deposition of $\mathrm{BiFe}_{3}, f$ ) surface topography after a total of $6 \mathrm{~nm} \mathrm{BiFeO}$. Line profile of $\mathrm{SrR} u \mathrm{O}_{3}$ surface and after each g) $\mathrm{PbTiO}_{3}$ and h) $\mathrm{BiFeO}_{3}$ deposition. i) $\mathrm{RMS}$ and average peak to peak height of the starting $\mathrm{SrRu} \mathrm{O}_{3}$ nanoislands and after each $\mathrm{PbTiO}_{3}$ and $\mathrm{BiFeO}_{3}$ deposition 
With the deposition of three more nanometers of $\mathrm{BiFeO}_{3}$ the $\mathrm{SrRuO}_{3}$ island pattern can no longer been seen in the surface topography. Most of the $\mathrm{BiFeO}_{3}$ surface is atomically smooth with a few deeper trenches that have not completely been filled-in yet. The differences between the growth of the $\mathrm{PbTiO}_{3}$ and the $\mathrm{BiFeO}_{3}$ are more clearly shown by looking at the line profiles of the surface and comparing the RMS and average peak to peak heights between the depositions. Line profiles from the starting $\mathrm{SrRuO}_{3}$ surface and after each $3 \mathrm{~nm}$ deposition are shown in Figure 5.8g for $\mathrm{PbTiO}_{3}$ and Figure 5.8h for $\mathrm{BiFeO}_{3}$. The profiles have been arbitrarily displaced by $5 \mathrm{~nm}$ from each other for visualization purposes. For the $\mathrm{PbTiO}_{3}$, Figure $5.8 \mathrm{~g}$, the three profiles are very similar to each other with the only slight difference being the islands tops becoming rounder with each deposition. For the $\mathrm{BiFeO}_{3}$, Figure 5.8h, the flattening of the surface in each successive deposition is very apparent. These observations are quantified by comparing the RMS and average peak to peak heights for the different surfaces, Figure 5.8i. For the $\mathrm{PbTiO}_{3}$ both the RMS and average peak to peak height remain constant at around 2.5 and $11 \mathrm{~nm}$ respectively. For the $\mathrm{BiFeO}_{3}$ the two surface parameters each decrease rapidly. The RMS goes from 2.25 to 1.43 to $0.22 \mathrm{~nm}$ while the peak to peak decreases from 7.67 to 6.66 to $1.26 \mathrm{~nm}$.

\subsection{Discussion}

From the results given above it is clear that the domain structure and growth dynamics of $\mathrm{PbTiO}_{3}$ and $\mathrm{BiFeO}_{3}$ grown on a patterned bottom electrode are very different. The $\mathrm{SrRuO}_{3}$ nanowires produced an alignment of both the $90^{\circ}$ and $180^{\circ}$ domains in the $\mathrm{PbTiO}_{3}$ film. From the TEM results, the nanowire edges act as nucleation sites for the creation of $a$ domains while along the nanowires a regular $90^{\circ}$ periodic domain structure was observed. The growth study of $\mathrm{PbTiO}_{3}$ showed that the film retained the initial 3D structure of the patterned electrode with most of the growth occurring from a lateral increase in the nanoislands size until the islands began to merge together. The $\mathrm{BiFeO}_{3}$ showed much less domain alignment with the underlying $\mathrm{SrRuO}_{3}$ nanowires. Only the area directly over the nanowires showed any preferential alignment in the nanowire direction. The growth studies revealed that $\mathrm{BiFeO}_{3}$ has a strong preference to grow atomically smooth surfaces by filling in the area between the nanostructures. As a result the original 3D pattern of the bottom electrode is quickly erased.

Taken together these results imply that how the material grows on a 3D surface is just as important as the $3 \mathrm{D}$ pattern in using a patterned bottom electrode to create periodic lateral boundary conditions to influence the domain structure of the ferroelectric. From the growth study of the $\mathrm{PbTiO}_{3}$ and $\mathrm{BiFeO}_{3}$ films two different growth modes are observed and shown schematically in Figure 5.9. 


\section{$\mathrm{PbTiO}_{3}$}

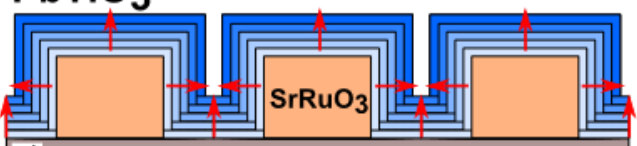

a)

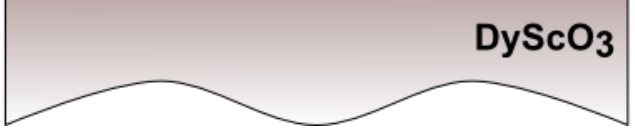

$\mathrm{BiFeO}_{3}$

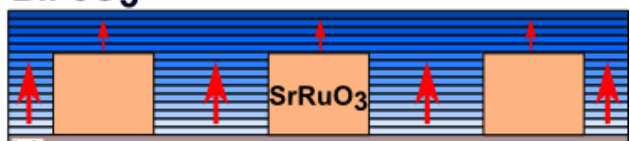

b)

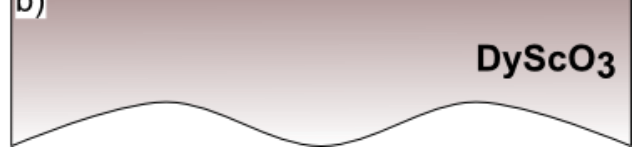

Figure 5.9 Schematic of the growth of the a) $\mathrm{BiFeO}_{3}$ and b) $\mathrm{PbTiO}_{3}$ films on nanostructures.

For the $\mathrm{PbTiO}_{3}$, Figure 5.9a, the arriving deposited material has an equal preference to nucleate and grow on both the $\mathrm{DyScO}_{3}$ substrate and the $\mathrm{SrRuO}_{3}$ islands. As a result, the $\mathrm{PbTiO}_{3}$ film grows at the roughly same rate on or off the $\mathrm{SrRuO}_{3}$ maintaining the initial pattern. For the $\mathrm{BiFeO}_{3}$, Figure 5.9b, there is a strong preference for the growth of the film to take place on the $\mathrm{DyScO}_{3}$ surface. This could be due to a much higher surface diffusivity of the $\mathrm{BiFeO} 3$ compared to $\mathrm{PbTiO} 3$ which will allow the arriving material to diffuse off of the nanowires to nucleate and grown over the $\mathrm{DyScO}$. As a result the $\mathrm{BiFeO}_{3}$ fills up the regions in-between the $\mathrm{SrRuO}_{3}$ until an atomically smooth film is produced. After this point the film continues to grow as a flat film with no influence of the $\mathrm{SrRuO}_{3}$ nanopattern.

These two different growth modes and the difference in the ferroelectric transition temperatures explain why the $\mathrm{PbTiO}_{3}$ showed a strong domain alignment with the nanowires and the $\mathrm{BiFeO}_{3}$ did not. For the $\mathrm{PbTiO}_{3}$ the film is grown at $600^{\circ} \mathrm{C}$ well above its $\mathrm{T}_{\mathrm{c}}$ of $490^{\circ} \mathrm{C}$ so the film is in a cubic state until the entire film is grown and the sample is cooled down to room temperature. The high aspect ratio of the $\mathrm{SrRuO}_{3}$ nanowires combined with the conformal coating by the growth mode of the $\mathrm{PbTiO}_{3}$ results in strain forming in the $\mathrm{PbTiO}_{3}$ film around the nanowires edges. When the film is cooled through the transition temperature and becomes ferroelectric $90^{\circ}$ domains form at the nanowires edges to relieve this stress resulting in the $90^{\circ}$ domains following the nanowires edges.

Another interesting aspect of the domain structure in the $\mathrm{PbTiO}_{3}$ on the nanowires is the lack of difference in the $180^{\circ}$ domain pattern of the films along the nanowires for regions of the film on or off the nanowires. The difference in electrical boundary conditions should result in a change in the domain pattern. The reason for this is most likely because of small $\mathrm{SrRuO}_{3}$ islands that form between the nanowires. Although there is still ferroelectric grown directly on the insulating substrate a significant region of the space in-between the nanowires is occupied by the $\mathrm{SrRuO}_{3}$ islands. These islands are not grounded but do provide screening to reduce the depolarizing field preventing a large difference in the electrical boundary conditions from developing. 
For the $\mathrm{BiFeO}_{3}$ the growth temperature of $600^{\circ} \mathrm{C}$ is below the ferroelectric transition temperature of $830^{\circ} \mathrm{C}$. This means that the $\mathrm{BiFeO}_{3}$ is in the ferroelectric state as the film is being grown. As a result, the domain structure is being set while the $\mathrm{BiFeO}_{3}$ is growing only on the $\mathrm{DyScO}_{3}$ substrate with no influence from the $\mathrm{SrRuO}_{3}$ structures. As the $\mathrm{BiFeO}_{3}$ fills in the $\mathrm{SrRuO}_{3}$ wires little to no strain forms around the nanowire edges. The combination of the growth mode and the high transition temperature means the nanowire pattern has little effect on the domain pattern in the ferroelectric film.

\subsection{Conclusions}

In summary thin films of $\mathrm{BiFeO}_{3}$ and $\mathrm{PbTiO}_{3}$ were grown on $\mathrm{SrRuO}_{3}$ nanowires and nanoislands. The two films were found to behave very differently in both their domain structure and surface morphology. $\mathrm{For}_{\mathrm{PbTiO}}$ the underlying nanowires were visible in the surface morphology and the $180^{\circ}$ and $90^{\circ}$ domains align with the nanowires. The opposite is true for $\mathrm{BiFeO}_{3}$ where very little domain alignment takes place and the film surface is atomically smooth. The results presented here are important for understanding the properties of miniaturization of ferroelectrics down to nanoscale components for standard and new electronic devices. At these dimensions not only are the intrinsic properties of the ferroelectric important but also extrinsic properties such as the growth mode of the material. However, this does not have to be a technological stumbling block. The ability to control the domain structure of a ferroelectric film by the use of a patterned bottom electrode opens up the possibility for novel domain structures beyond what is possible with traditional strain engineering, as long as the growth kinetics permit the desired boundary conditions to be transferred to the ferroelectric film. This creates the possibility to further tailor the properties of the ferroelectric film to the specific device application. 


\section{References}

[1] O. Auciello, C. M. Foster, and R. Ramesh, "Processing technologies for ferroelectric thin films and heterostructures," Annual Review of Materials Science, vol. 28, pp. 501-531, 19981998.

[2] O. Auciello, J. F. Scott, and R. Ramesh, "The physics of ferroelectric memories," Physics Today, vol. 51, pp. 22-27, Jul 1998.

[3] P. Muralt, "Ferroelectric thin films for micro-sensors and actuators: a review," Journal of Micromechanics and Microengineering, vol. 10, pp. 136-146, Jun 2000.

[4] R. Ramesh, S. Aggarwal, and O. Auciello, "Science and technology of ferroelectric films and heterostructures for non-volatile ferroelectric memories," Materials Science \& Engineering R-Reports, vol. 32, pp. 191-236, Apr 162001.

[5] J. F. Scott and C. A. P. Dearaujo, "Ferroelectric Memories," Science, vol. 246, pp. 1400-1405, Dec 151989.

[6] K. Uchino, Ferroelectric Devices: Marcel Dekker, 2000.

[7] N. Setter, "Electroceramics: looking ahead," Journal of the European Ceramic Society, vol. 21, pp. 1279-1293, 20012001.

[8] N. Setter and R. Waser, "Electroceramic materials," Acta Materialia, vol. 48, pp. 151 178, Jan 12000.

[9] D. L. Polla and L. F. Francis, "Processing and characterization of piezoelectric materials and integration into microelectromechanical systems," Annual Review of Materials Science, vol. 28, pp. 563-597, 19981998.

[10] N. A. Pertsev, A. G. Zembilgotov, and A. K. Tagantsev, "Effect of mechanical boundary conditions on phase diagrams of epitaxial ferroelectric thin films," Physical Review Letters, vol. 80, pp. 1988-1991, Mar 21998.

[11] N. A. Pertsev, A. K. Tagantsev, and N. Setter, "Phase transitions and strain-induced ferroelectricity in SrTiO3 epitaxial thin films," Physical Review B, vol. 61, pp. R825R829, Jan 12000.

[12] Y. L. Li, S. Choudhury, Z. K. Liu, and L. Q. Chen, "Effect of external mechanical constraints on the phase diagram of epitaxial PbZr1-xTixO3 thin films thermodynamic calculations and phase-field simulations," Applied Physics Letters, vol. 83, pp. 1608-1610, Aug 252003.

[13] K. S. Lee, J. H. Choi, J. Y. Lee, and S. Baik, "Domain formation in epitaxial $\mathrm{Pb}(\mathrm{Zr}, \mathrm{Ti}) \mathrm{O}-3$ thin films," Journal of Applied Physics, vol. 90, pp. 4095-4102, Oct 15 2001.

[14] S. Venkatesan, B. J. Kooi, J. T. M. De Hosson, A. H. G. Vlooswijk, and B. Noheda, "Substrate influence on the shape of domains in epitaxial $\mathrm{PbTiO}(3)$ thin films," Journal of Applied Physics, vol. 102, Nov 152007. 
[15] A. H. G. Vlooswijk, B. Noheda, G. Catalan, A. Janssens, B. Barcones, G. Rijnders, et al., "Smallest 90 degrees domains in epitaxial ferroelectric films," Applied Physics Letters, vol. 91, Sep 102007.

[16] I. Vrejoiu, A. Morelli, F. Johann, and D. Biggemann, "Ordered 180 degrees ferroelectric domains in epitaxial submicron structures," Applied Physics Letters, vol. 99, Aug 222011.

[17] B. Kuiper, J. L. Blok, H. J. W. Zandvliet, D. H. A. Blank, G. Rijnders, and G. Koster, "Self-organization of SrRuO3 nanowires on ordered oxide surface terminations," Mrs Communications, vol. 1, pp. 17-21, Nov 2011.

[18] H. W. Jang, D. Ortiz, S.-H. Baek, C. M. Folkman, R. R. Das, P. Shafer, et al., "Domain Engineering for Enhanced Ferroelectric Properties of Epitaxial (001) BiFeO3 Thin Films," Advanced Materials, vol. 21, pp. 817-+, Feb 162009.

[19] S. Farokhipoor and B. Noheda, "Conduction through 71 degrees DomainWalls in BiFeO3 Thin Films," Physical Review Letters, vol. 107, Sep 142011. 


\section{Chapter 6}

\section{$\mathrm{BiFeO}_{3}$ Domain Wall}

\section{Conductivity using $\mathrm{SrRuO}_{3}$}

\section{Nanowires}

\subsection{Introduction}

In the previous chapter $\mathrm{SrRuO}_{3}$ nanowires were used as a means to locally modulate the boundary conditions of a ferroelectric film changing these boundary conditions across the film creating new domain structures. In this chapter the same nanowires are used to locally probe the properties of a ferroelectric film particularly the properties of domain walls. The nanowires serve as bottom electrodes for ferroelectric switching and electrical conductivity measurements in $\mathrm{BiFeO}_{3}$ thin films. In this way the nanowires serve as a practical tool to access local properties which are useful for fundamental studies and possibly practical devices.

Domains in ferroic materials have been studied extensively for many years. The study of the domain walls, however, is much less developed due to several reasons. First, the size of ferroelectric domain walls is extremely small, down to a single unit cell in width for some ferroelectric domains, making experimental studies challenging. Recent advances in scanning probe techniques and transmission electron microscopy are finally providing researchers tools to examine domain walls [1-4]. Second, for practical purposes the properties of domains have generally been regarded as unimportant since the volume fraction of domain walls is generally orders of magnitude smaller than the volumes of the domains themselves making any response from the domain walls a miniscule component of the response from the entire material. As has been shown in the previous chapters improved thin film growth techniques allow the fabrication of sub-micron thick films where the domain wall density can become significant making response from the domain walls no longer negligible [5]. 
One of the most striking examples of new phenomena found to exist at domain walls is domain wall conductivity in $\mathrm{BiFeO}_{3}$. Domain wall conductivity was first reported in 2009 by Siedel et al. [6] In their study different domain walls were written by the application of a DC electric field using an AFM tip. Subsequent cAFM measurements revealed conductivity at $109^{\circ}$ and $180^{\circ}$ domain walls while the $71^{\circ}$ domain walls remained insulating. After this initial report rapid progress took place identifying domain wall conductivity in as-grown $180^{\circ}$ and $109^{\circ} \mathrm{BiFeO}_{3}$ domain walls [7], as-grown $71^{\circ} \mathrm{BiFeO}_{3}$ domain walls [8], $180^{\circ}$ domain walls in PZT [9], and in domain walls of rare earth manginates [10]. Furthermore, the conductivity at these domain walls has been shown to go beyond simple rigid conductors. Maksymovych et al. have shown that domain wall conductivity can be enhanced and even tuned showing memristive like behavior [11]. All of these observations have led to many proposed mechanisms for the conduction at ferroelectric domain walls from accumulation of oxygen vacancies to due local strain around domain walls [8], to band gap lowering from changes in the polarization normal to the domain wall [6], to charging of the domain wall due to slight inclination of the domain wall $[12,13]$.

In all of these studies the goal of the investigation is to determine the conduction mechanism from the electronic properties of the domain wall and then develop a physical model of the conduction from this mechanism. However, one necessary piece of information is missing which is important to distinguish the conduction mechanism; how the conductivity of the domain wall changes with length. As shown in Chapter 1 some of the different conduction mechanisms have similar I-V characteristics which can be easily distinguished by looking at how the current evolves the length. These studies cited above are limited to examining the conduction on only one length scale, the thickness of the film. Several films of different thicknesses can be grown but due to the time consuming nature of preparing new films only a few thicknesses are examined making trends difficult to identify. The use of the $\mathrm{SrRuO}_{3}$ nanowires provides a perfect solution to these problems and a schematic of the advantages of this experimental setup is shown in Figure 6.1. First, in only one sample we can easily identify the difference in conduction between domains that intersect a conducting wire and those that only make contact with the insulating substrate. In addition, domains that run perpendicular to the underlying nanowires can be used to examine how the conductivity evolves on length scale beyond just the film thickness with domain walls either running off of a nanowire or a domain wall that connects two adjacent nanowires. On these domain walls the evolution of the conductivity with domain wall length can be measured continuously from right off on nanowire all the way to the next nanowire. Preforming I-V curves on different categories of domain walls (on, off, and across wires) providing more information on the electronic properties of the domain walls of $\mathrm{BiFeO}_{3}$ thin films. 


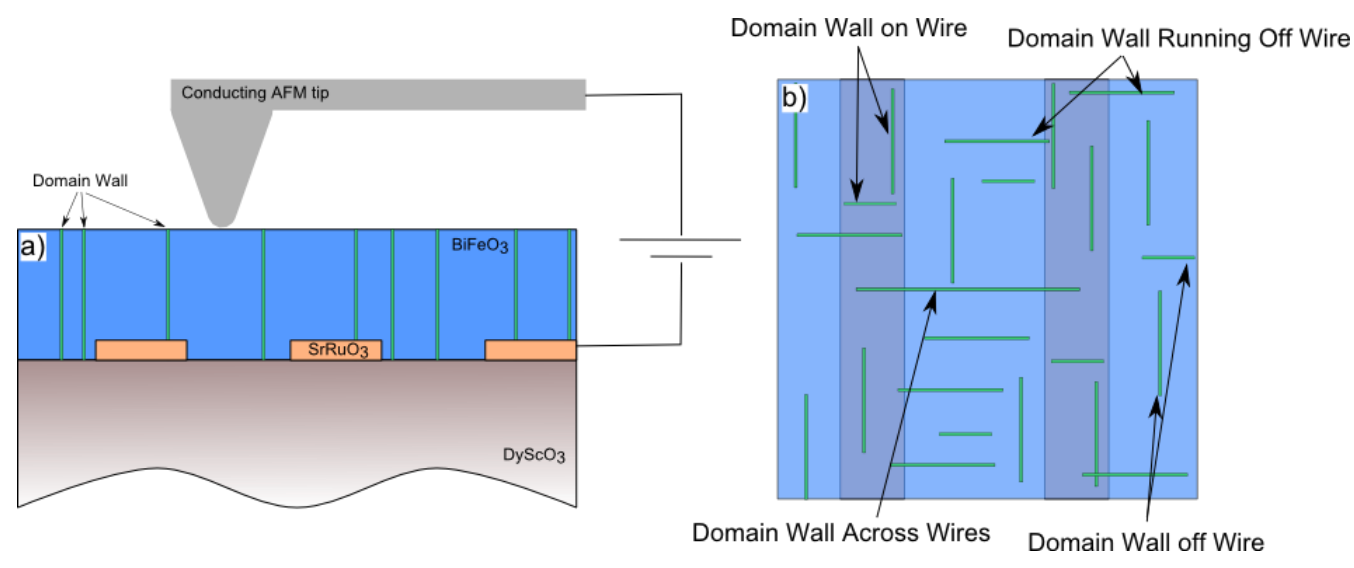

Figure 6.1 Experiment schematic for study of domain wall conductivity on local switching dynamics, a) side view with AFM tip and b) top view showing the different categories of domain walls.

\subsection{Experimental}

The $25 \mathrm{~nm} \mathrm{BiFeO}_{3}$ thin film on $5 \mathrm{~nm} \mathrm{SrRuO}_{3}$ nanowires from Chapter 4 was used to investigate the domain wall conductivity at new length scales. The atomically smooth surface of the $\mathrm{BiFeO}_{3}$ film is ideal for scanning probe investigation of local electronic properties eliminating cross talk with the surface topography. Examination of the electrical properties of the domain walls was done by conducting AFM (cAFM). Using an AFM tip to measure the current allows the local electrical properties to be measured on the nanoscale with currents ranging from picoamps to microamps. By switching between the cAFM and PFM modes it is possible to map the ferroelectric domains, determine the domain wall type and examine their electrical properties. The $\sim 325 \mathrm{~nm}$ nanowire periodicity will allow the study of domain wall conductivity over length scales not typically investigated as most studies investigate films with thicknesses of $100 \mathrm{~nm}$ or less. Current-voltage curves were performed on and off the nanowires to obtain more information on the electrical properties of the film in the two different regions.

\subsection{Results}

The topography and cAFM conductivity map of the $25 \mathrm{~nm} \mathrm{BiFeO} 3$ thin film on $5 \mathrm{~nm}$ $\mathrm{SrRuO}_{3}$ wires is shown in Figure 6.2 for two length scales $(6.5 \mu \mathrm{m}$ and $2 \mu \mathrm{m})$. The cAFM was done with $-4 \mathrm{~V}$ DC bias applied to the AFM tip with $\mathrm{SrRuO}_{3}$ wires grounded. Therefore regions of high conductivity will appear as dark and low conductivity regions will appear light. Figure 6.2a shows the surface topography of the $\mathrm{BiFeO}_{3}$ showing the atomically smooth surface with no indication of the underlying nanowires. However, in the cAFM image, Figure $6.2 \mathrm{~b}$, regular lines of high conductivity are visible. These high conductivity regions match the 
size and separation of the $\mathrm{SrRuO}_{3}$ nanowires. Closer examination of these higher conductive regions of the $\mathrm{BiFeO}_{3}$ over the $\mathrm{SrRuO}_{3}$ nanowires shows that the conductivity is not uniform over the nanowires. There is a fine structure to the conductivity that is more visible after zooming on a region of the film with only a few nanowires. The zoomed in topography and cAFM images are shown in Figure 6.2c and 6.2d respectively. Over the nanowires the high conductivity is only observed in very localized stripes while the rest of the surface over the nanowires has the same low conductivity as the $\mathrm{BiFeO}_{3}$ not over the nanowires.
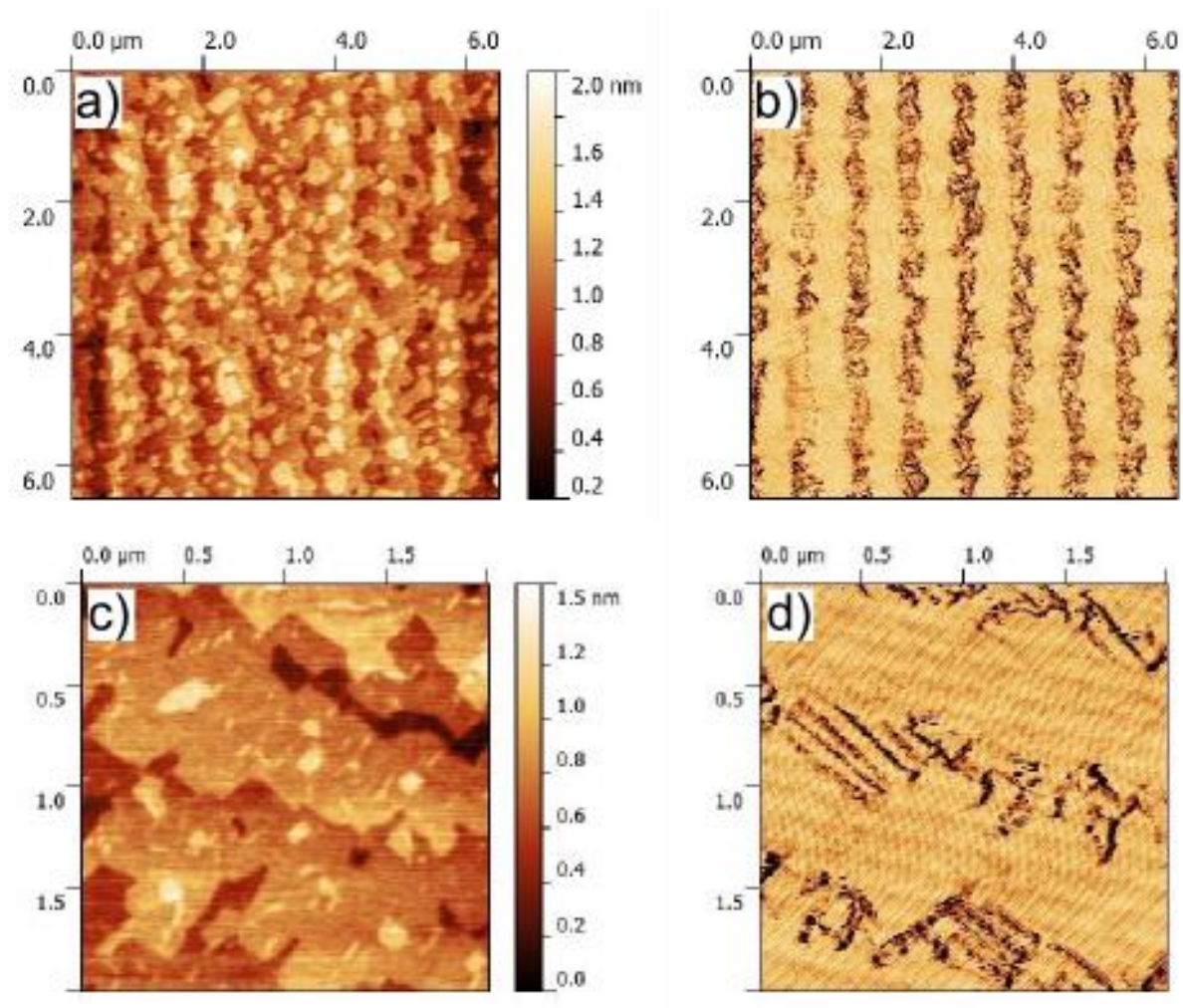

Figure 6.2 Topography (a,c) and cAFM (b.d) images of $25 \mathrm{~nm}$ of $\mathrm{BiFe}_{3}$ on $5 \mathrm{~nm} \mathrm{SrRuO}_{3}$ nanowires

The narrow shape of the high conductivity regions suggests that this conductivity is due to domain walls. Frist we must confirm that the domain walls are responsible for the observed conductivity by observing the domain structure through subsequent PFM images done in the same regions to visualize the ferroelectric domains. The topography, PFM and cAFM images are shown in Figure 6.3. The surface topography of the area used for these measurements is shown in Figure 6.3a. Figure $6.3 \mathrm{~b}$ and $\mathrm{c}$ show the out of plane PFM amplitude and phase respectively confirming that the majority of the film has one out of plane 
polarization orientation with some small regions of opposite polarization. The in-plane amplitude and phase images are shown in Figure 6.3e and $\mathrm{f}$ showing that all four in-plane polarization orientations are present. Considering the in-plane and out of plane PFM images together $\mathrm{BiFeO} 3$ film consists of 4 out of 8 possible polarization orientations. Analysis of the domain orientation reveals that the domain walls are $71^{\circ}$ walls. The cAFM image is shown in Figure $6.3 \mathrm{~d}$. There is a strong correlation between the position of the domain walls and the high conductivity regions in the CAFM image and the clearest examples of this correlation are circled in the in-plane PFM and cAFM images. Looking closely at the large green circle there are four distinct lines of high conductivity pointed out with the red arrows and labeled with roman numerals from top right to bottom left. The red arrows in the in-plane PFM amplitude and phase image at the same position on the substrate clearly correspond with the four domain walls.
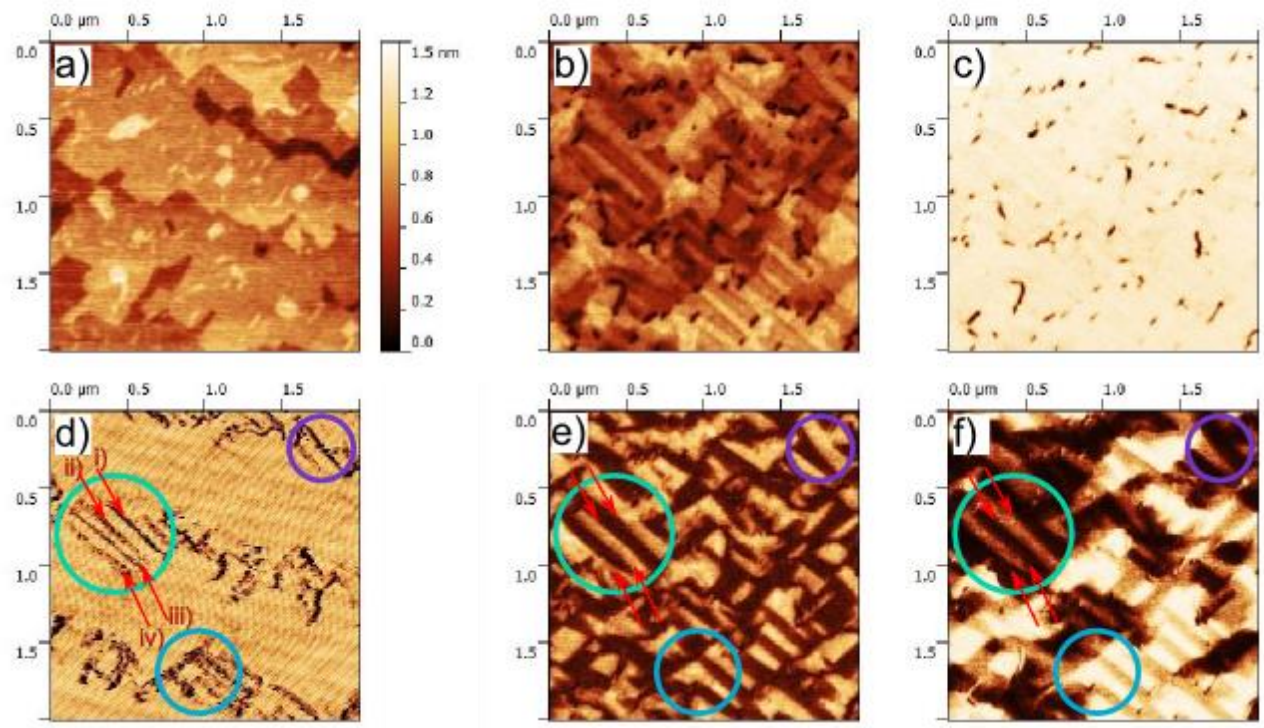

Figure 6.3 a) Surface topography, b) out of plane PFM amplitude c) out of plane PFM phase, d) current e) in-plane PFM amplitude and f) in-plane PFM phase showing $71^{\circ}$ domain wall conductivity over the $\mathrm{SrR}_{\mathrm{R}} \mathrm{O}_{3}$ nanowires

Positioning a conductive AFM tip at different locations on the film I-V curves were measured sweeping the voltage applied to the tip from $-5 \mathrm{~V}$ to $2 \mathrm{~V}$ (forward sweep) and back (backward sweep). The resulting curves for three domain walls on (positions 1,2,3) and three domain walls off (position 4,5,6) the SrRuO3 wire are shown in Figure 6.4. In all cases the current is only observed at negative applied voltage. For positions on the wire the current at $5 \mathrm{~V}$ is approximately 7 times larger than for positions off the nanowires ( $35 \mathrm{pA}$ to $5 \mathrm{pA}$ ). 
Furthermore, on the nanowires there is a significant difference between the forward and backward voltage sweeps. In the forward sweep there are several distinct jumps in the current. The size and number of current jumps changes depending on the position. Position 1 has 3 distinct jumps; position 3 has only 2 jumps while position 2 has many small current jumps. In between these jumps the current is mostly constant. On the backwards sweep these jumps disappear and an exponential increase in the current is observed. These jumps are also not observed if additional voltage sweeps are performed. For additional voltage sweeps the forward sweep matches the backward sweep. This behavior is also not observed on for the I$\mathrm{V}$ curves taken at positions off the nanowires.
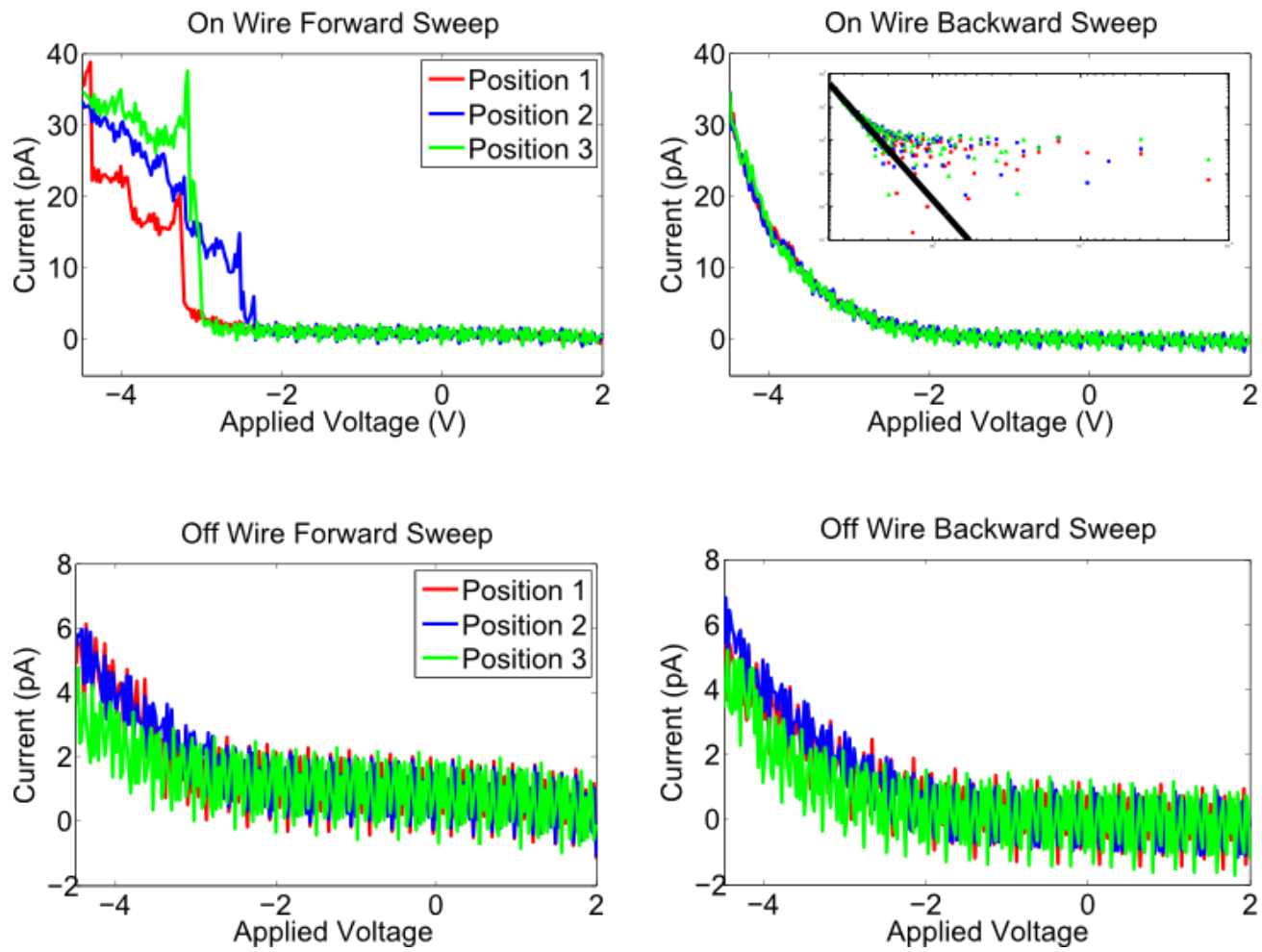

Figure 6.4 IV curves from $-4.5 \mathrm{~V}$ to $2 \mathrm{~V}$ on and off wires at three different positions for each region on and off the nanowires. The inset is a log-log plot of the data with an exponential fit IaV $V^{-5}$

To study how the domain wall conductivity changes with distance it is necessary to first to have a long domain wall that runs perpendicular to the nanowires. In Figure 6.3 none of the domain walls fulfill this criterion. Attempts at writing such a domain wall by applying a DC bias with the AFM tip were not successful. However, a domain wall running across two nanowires was found and is shown in Figure 6.5 with the corresponding PFM images. Figure 
6.5a shows the surface topography; again showing an atomically smooth surface with unit cell steps.

The two underlying nanowires are visualized by domain wall conductivity in the cAFM image in Figure 6.5b.

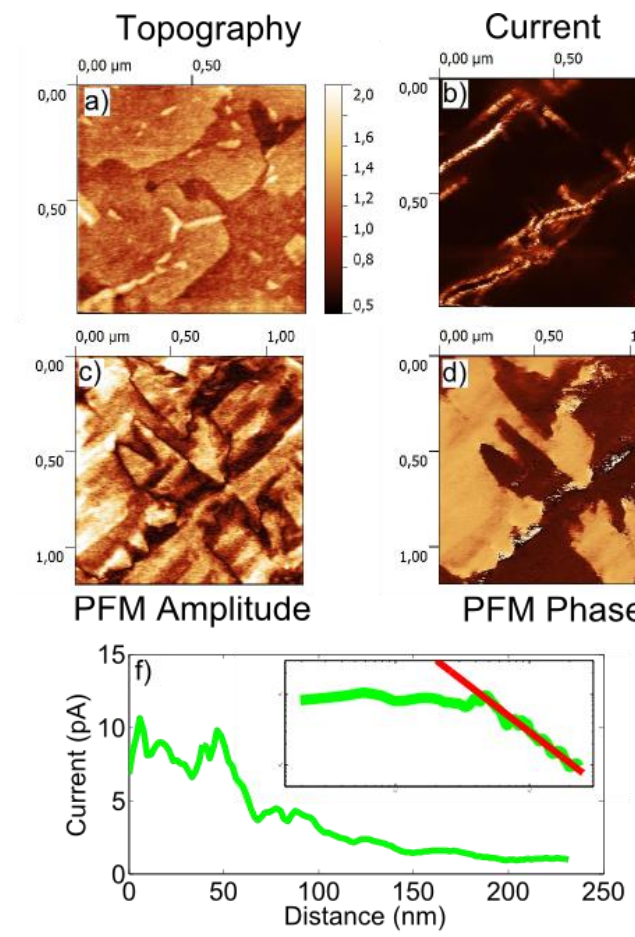

Current profile upper trace
Current and PFM amplitude

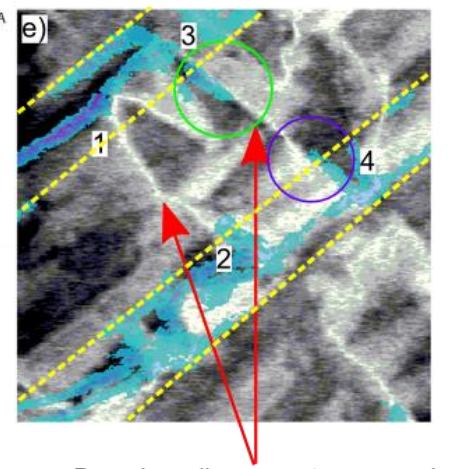

Domain walls across two nanowires

Current profiles along domain wall from each end

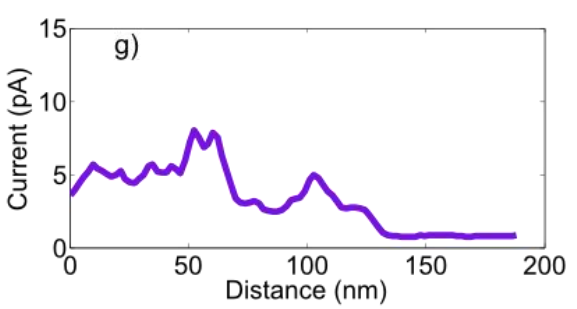

Current profile lower trace

Figure 6.5 a) surface topography b) (AFM c) in-plane PFM amplitude d) in-plane PFM phase e) PFM amplitude and $C A F M$ images overlaid on each other and ff,g) current profile along domain wall across two nanowires upper and lower trace respectively. Inset shows the fit of Iad $-{ }^{1.5}$.

In the PFM amplitude and phase image, Figure $6.5 \mathrm{c}$ and $\mathrm{d}$ respectively, several $71^{\circ}$ domain are present many of which are running perpendicular to the nanowires. Correcting for AFM drift and overlaying the PFM amplitude and cAFM images, Figure 6.5e, we can see there are two domain walls that run across adjacent nanowires and are identified with red arrows. The approximate positions of the nanowires are outlined with dashed yellow lines. For the domain wall on the left there is no conductivity extending off the upper nanowire (domain wall end 1). As the domain wall extends off the lower nanowire the conductivity stops abruptly (domain wall end 2). The domain wall on the right side shows conductivity extending off of both the upper and lower nanowires. Current profiles along this domain wall from the upper 
and lower nanowire are plotted to get more information about the behavior of the conductivity. From the upper nanowire the current along the nanowire decreases continuously from $\sim 10 \mathrm{pA}$ on the nanowire to $1 \mathrm{pA}$ between the two nanowires (domain wall end 3). Fitting the decrease of the current with distance from the nanowire shows that $\mathrm{I} \alpha \mathrm{d}-1.5$. From the lower nanowire the conductivity decreases in two clear steps of $4.5 \mathrm{pA}$ and $4 \mathrm{pA}$ at around 60 $\mathrm{nm}$ and $125 \mathrm{~nm}$ respectively down to $1 \mathrm{pA}$ in the middle of the nanowires (domain wall end 4). No observations were found where a domain wall retained high conductivity throughout the length of the domain wall as it extended off a nanowire.

\subsection{Discussion}

The cAFM images and I-V curves of the $71^{\circ}$ domain walls on, off, and across the nanowires provides a lot of information about their electronic properties. First, current is only measured in the film when a domain wall intersects a nanowire at the bottom interface. If a domain wall is only in contact with the insulting substrate then there is an open circuit and no current will flow through the tip into the current amplifier. When a domain wall is in contact with a nanowire then the circuit is complete and current is able to flow through the tip and current amplifier. The fact that a closed circuit is need to observe domain wall conductivity proves that the conductivity is not due to any transient phenomena such as switching current or ionic motion.

The jumps in current during the first sweep of the voltage in the I-V curves was unexpected and indicate that some process is taking place driven by the initial applied electric field. The two possible candidates are either motion of the domain wall under the tip or migration of defects; most likely oxygen vacancies. The more likely answer is motion of the domain wall. Migration of defects should not result in large steps as in position 1 as this would be a more continuous process as vacancies accumulate at the domain walls. However, discrete motion of the domain wall could produce jumps in the conductivity. Although on the surface there does not appear to be any change in the domain wall position with PFM images after the $\mathrm{I}-\mathrm{V}$ curves this does not rule out motion of the domain wall somewhere buried in the thickness of the film.

For the two domain walls that run across adjacent four different behaviors are observed as the four ends of the domain walls run off the nanowires. In the case of domain wall edge 1 no conductivity was observed off the nanowire. However it is not clear that the domain wall actually extends over the nanowire as the domain wall curves right around right near the nanowire edge and most likely indicates that the domain wall just misses the nanowire. The other three domain wall ends clearly intersect the underlying nanowire. The absence of conductivity in the center of the wire in these cases indicates that the conduction of the domain wall is bulk limited. Fitting the exponential decrease in the conductivity for domain 
wall edge 4 gave an exponent of -1.5. This exponent does not fit with either of the bulk limited conduction mechanism for semiconductors which should be -3 for space charge limited conduction and -0.5 for Poole-Frenkel hopping. The different behaviors for observed for the domain wall ends running of the nanowires indicate that several processes could be taking place. These processes are most likely related to different defects along the domain wall either from Poole-Frenkel hopping between oxygen vacancies which accumulate around the domain wall while the discrete jumps in conductivity could be from movement or deviations in the domain below the surface of the film.

\subsection{Conclusions}

In summary, this chapter has examined the conductivity of as-grown $71^{\circ}$ domain walls in a $25 \mathrm{~nm}$ thick $\mathrm{BiFeO}_{3}$ film. The use of $\mathrm{SrRuO}_{3}$ nanowires instead of a continuous bottom electrode has enabled the study of the domain wall conductivity of domain walls on the wire, off the wire and running across wires. This system allows easy study of the distance depends of domain wall conductivity when a domain wall runs across the nanowires. The lack of any observable domain wall conductivity for domain walls that do not intersect a nanowire prove that the domain wall conductivity is not due to any transient phenomena such as switching currents or ionic motion. The fact that the conductivity decreases with domain wall length as the domain walls run off a nanowire indicates that the conduction mechanism is bulk limited. However, the different behavior of the decrease in current with distance (exponentially or with discrete jumps) and the exponent of -1.5 does not fit in with any of the traditional bulk limited conduction mechanisms for semiconductors. This inconsistency along with the lack of temperature data makes a concrete determination of the conduction mechanism difficult to determine. 


\section{References}

[1] C. L. Jia, M. Lentzen, and K. Urban, "Atomic-Resolution Imaging of Oxygen in Perovskite Ceramics," Science, vol. 299, pp. 870-873, February 7, 20032003.

[2] C.-L. Jia, S.-B. Mi, K. Urban, I. Vrejoiu, M. Alexe, and D. Hesse, "Atomic-scale study of electric dipoles near charged and uncharged domain walls in ferroelectric films," Nature Materials, vol. 7, pp. 57-61, Jan 2008.

[3] L. M. Eng, "Nanoscale domain engineering and characterization of ferroelectric domains," Nanotechnology, vol. 10, pp. 405-411, Dec 1999.

[4] T. Tybell, P. Paruch, T. Giamarchi, and J. M. Triscone, "Domain Wall Creep in Epitaxial Ferroelectric $\mathrm{Pb}\left(\mathrm{Zr}_{-}\{0.2\} \mathrm{Ti}_{-}\{0.8\}\right) \mathrm{O}_{-}\{3\}$ Thin Films," Physical Review Letters, vol. 89, p. 097601, 08/09/ 2002.

[5] G. Catalan, J. Seidel, R. Ramesh, and J. F. Scott, "Domain wall nanoelectronics," Reviews of Modern Physics, vol. 84, pp. 119-156, 2012.

[6] J. Seidel, L. W. Martin, Q. He, Q. Zhan, Y. H. Chu, A. Rother, et al., "Conduction at domain walls in oxide multiferroics," Nat Mater, vol. 8, pp. 229-34, Mar 2009.

[7] J. Seidel, P. Maksymovych, Y. Batra, A. Katan, S. Y. Yang, Q. He, et al., "Domain Wall Conductivity in La-Doped BiFeO_\{3\}," Physical Review Letters, vol. 105, 2010.

[8] S. Farokhipoor and B. Noheda, "Conduction through $71^{\circ}$ Domain Walls in BiFeO_\{3\} Thin Films," Physical Review Letters, vol. 107, 2011.

[9] J. Guyonnet, I. Gaponenko, S. Gariglio, and P. Paruch, "Conduction at domain walls in insulating $\mathrm{Pb}(\mathrm{Zr0} .2$ Ti0.8)O3 thin films," Adv Mater, vol. 23, pp. 5377-82, Dec 1 2011.

[10] W. D. Wu, Y. Horibe, N. Lee, S. W. Cheong, and J. R. Guest, "Conduction of Topologically Protected Charged Ferroelectric Domain Walls," Physical Review Letters, vol. 108, Feb 2012.

[11] P. Maksymovych, J. Seidel, Y. H. Chu, P. Wu, A. P. Baddorf, L. Q. Chen, et al., "Dynamic conductivity of ferroelectric domain walls in $\mathrm{BiFeO}(3), "$ Nano Lett, vol. 11, pp. 1906-12, May 112011.

[12] R. K. Vasudevan, A. N. Morozovska, E. A. Eliseev, J. Britson, J. C. Yang, Y. H. Chu, et al., "Domain wall geometry controls conduction in ferroelectrics," Nano Lett, vol. 12, pp. 5524-31, Nov 142012.

[13] A. N. Morozovska, "Domain Wall Conduction in Ferroelectrics," Ferroelectrics, vol. 438, pp. 3-19, 2012. 


\section{Chapter 7}

\section{$\mathrm{PbTiO}_{3}$ on $\mathrm{Ca}_{2} \mathrm{Nb}_{3} \mathrm{O}_{10}$}

\section{Nanosheets}

\subsection{Introduction}

Widespread research into nanoscale ferroelectrics did not start until the end of the 1990s with the development of several techniques capable of producing ferroelectric objects with dimensions on the nanoscale. Top down approaches were commonly used to fabricate ferroelectric nanostructures allowing precise control over the size, shape and position of the ferroelectric objects. Using Focused Ion Beam (FIB) milling to remove material from a thin ferroelectric capacitor stack nanocapacitors of $\mathrm{Pb}\left(\mathrm{Nb}_{0.04} \mathrm{Z}_{0.28} \mathrm{Ti}_{0.68}\right) \mathrm{O}_{3}$ [1] and $\mathrm{SrBi}_{2} \mathrm{Ta}_{2} \mathrm{O}_{9}$ [2] down to $70 \mathrm{~nm}$ by $70 \mathrm{~nm}$ were fabricated. Initially these islands did not show any piezoresponse. However, post annealing of the structures enabled recovery of the piezoresponse. Further research revealed that the piezoresponse is increased for $\mathrm{PbZr}_{0.2} \mathrm{Ti}_{0.8} \mathrm{O}_{3}$ nanoislands compared to a continuous film. This increase in the piezoresponse measured by scanning force microscopy was attributed to reduced substrate clamping allowing movement of $90^{\circ}$ domain walls [3]. Similar studies were also done using electron beam lithography creating nanoislands of $\mathrm{PbZr}_{0.4} \mathrm{Ti}_{0.6} \mathrm{O} 3$ down to $100 \mathrm{~nm}$ in diameter [4]. High piezoresponse measured with scanning force microscopy was observed for islands with lateral sizes less than $200 \mathrm{~nm}$. The measured increase was attributed to a change in domain structure with a reduction in the number $a$ domains in the nanoislands compared to continuous films. The a domains, having their polarization lying parallel to the top surface do not contribute to the out of plane piezoresponse which is measured using the scanning force microscopy technique. For small lateral dimensions the stress in the ferroelectric nanoislands is relaxed, as the island is no longer clamped to the rest of the ferroelectric film, eliminating the need to form $a$ domains. As a result a greater percentage of the nanoisland is in the $c$ domain state providing more contribution to the measured piezoresponse.

A major concern for top down fabrication methods is the damage to the ferroelectric either directly to the ferroelectric lattice during removal of unwanted material or contamination 
from resputtering. In order to avoid these problems several bottom up approaches have been utilized to study nanoscale ferroelectricity. Chemical solution deposited of $\mathrm{PbTiO}_{3}$ nanograins were found to lose their ferroelectricity below 20nm [5]. However, a similar study this time using metalorganic chemical vapor deposition showed $\mathrm{PbTiO}_{3}$ remained ferroelectric below lateral dimensions of $20 \mathrm{~nm}$ [6]. As with top down approaches the piezoresponse of CSD grown $\mathrm{PbTiO}_{3}$ nanoislands was found to increase compared to a thin film and attributed to a reduction of $a$ domains and relaxation of substrate clamping with decreasing lateral sizes [7]. The drawbacks to bottom up approaches are that there is much less control over size, shape, and position of the ferroelectric objects. This can make their characterization more difficult. In addition it is difficult to fabricate a wide range of sizes in a single sample. Therefore, several samples need to be made to extract size effect trends which are time consuming.

In this chapter we will investigate ferroelectric size effects using a bottom up approach by growing $\mathrm{PbTiO}_{3}$ thin films on $\mathrm{Ca}_{2} \mathrm{Nb}_{3} \mathrm{O}_{10}$ (CNO) nanosheets on a silicon substrate. The $\mathrm{CNO}$ nanosheets act as a seed layer for epitaxial growth of the $\mathrm{PbTiO}_{3}$. $\mathrm{PbTiO}_{3}$ grown off the nanosheets on the silicon will have a polycrystalline structure and the difference between the polycrystalline and epitaxial growth is easily differentiated by the surface morphology. As a result nanoscale epitaxial $\mathrm{PbTiO}_{3}$ objects having the same size and shape of the initial $\mathrm{CNO}$ nanosheets are grown from the bottom up. Using this technique several drawbacks from top down and traditional bottom up techniques are avoided. First, being a bottom up technique there is no removal of material or other processing steps eliminating damage to the patterning of ferroelectric nanostructures. Also, no post processing is required after the deposition of the $\mathrm{PbTiO}_{3}$ allowing the films to be characterized in situ eliminating contamination from the atmosphere. The size of the ferroelectric nanostructure is determined by the size of the initial nanosheets which range from $1.5 \mu \mathrm{m}$ down to $100 \mathrm{~nm}$. Therefore, unlike other bottom-up approaches, which usually produce uniformly sized nanoscale ferroelectrics, a wide range of ferroelectric sizes can be studied in one sample. Furthermore, the nanosheet density changes from no nanosheets to sparse separate nanosheets to tightly packed nanosheets. This allows the measurement of the ferroelectric objects that are tightly packed and separate which is used to determine if neighboring ferroelectric objects influence each other. Growing $\mathrm{PbTiO}_{3}$ on nanosheets ranging in lateral sizes from $1.5 \mu \mathrm{m}$ to $100 \mathrm{~nm}$ it should be possible to identify at what lateral dimension the piezoresponse starts to increases, as the lateral dimensions of the nanosheets are in the same length scale as the observation of increased piezoresponse in literature. In addition, using piezoresponse microscopy to image the domain structure the mechanism of the increased piezoresponse can be identified whether it is due to a reduction of $a$ domains, which will be visible in the in-plane PFM response, or other mechanisms. 


\subsection{Experimental}

$\mathrm{PbTiO}_{3}$ films of $30 \mathrm{~nm}$ were grown on $\mathrm{Ca}_{2} \mathrm{Nb}_{3} \mathrm{O}_{10}$ nanosheets to investigate the influence of size effects on the properties of $\mathrm{PbTiO}_{3}$. The $\mathrm{Ca}_{2} \mathrm{Nb}_{3} \mathrm{O}_{10}$ nanosheets were formed by exfoliation of a parent compound of $\mathrm{KCa}_{2} \mathrm{Nb}_{3} \mathrm{O}_{10}$ which results in the nanosheets suspended in an aqueous solution [8]. After exfoliation single atomic layer CNO nanosheets are suspended in solution. These nanosheets have perovskite structure with $\mathrm{NbO}_{6}$ octahedra with a lattice parameter of $3.86 \AA$ which provides a pretty good lattice match with $\mathrm{SrTiO}_{3}$ ($1.16 \%), \mathrm{SrRuO}_{3}(-1.7 \%)$ and $\mathrm{PbTiO}_{3}(-1.15 \%)$. From suspension the nanosheets were then deposited on a silicon wafer by Langmuir-Blodgett deposition. Silicon was chosen as a substrate material because it is cheap, able to withstand the deposition conditions for $\mathrm{PbTiO}_{3}$ growth and because there should be an obvious difference between the $\mathrm{PbTiO}_{3}$ grown on silicon and the nanosheets. On the silicon the $\mathrm{PbTiO}_{3}$ should be polycrystalline due to growth on the native amorphous silicon oxide layer while on the nanosheets the $\mathrm{PbTiO}_{3}$ should be more ordered and hopefully single crystalline. Empirically, a thin $\mathrm{SrTiO}_{3}$ layer of $5 \mathrm{~nm}$ was found to improve the growth of the $\mathrm{SrRuO}_{3}$ on the nanosheets. A $10 \mathrm{~nm} \mathrm{SrRuO}$ layer was used as a bottom electrode for the PFM measurements.

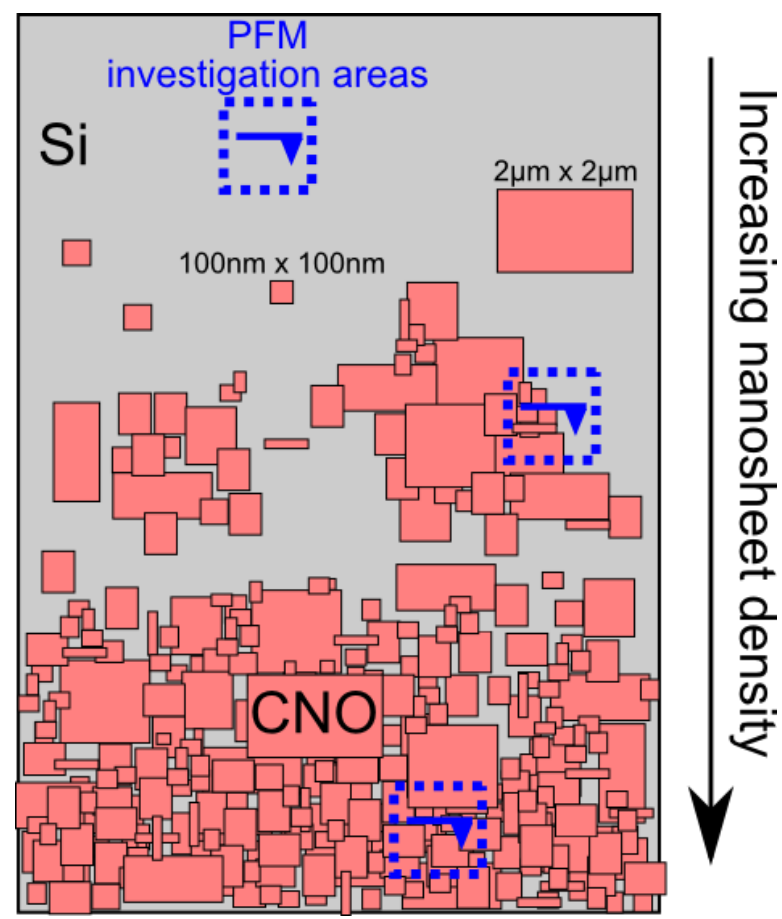

Figure 7.1 Schematic of the samples used to grow $\mathrm{PbTiO}_{3}$ nanoislands showing the multitude of nanosheets sizes and densities in a single sample. 
Shown schematically in Figure 7.1 each sample contains a variety of nanosheets sizes from $1.5 \mu \mathrm{m}$ to $100 \mathrm{~nm}$ and has regions with different nanosheet densities ranging from individually separated nanosheets to dense packing of the nanosheet with $\sim 97 \%$ coverage.

This range of nanosheet sizes and densities allows direct comparison of size effects in a single sample. PFM is used to investigate the piezoresponse and ferroelectric properties of the $\mathrm{PbTiO}_{3}$ due to this techniques high spatial resolution and ability to image the topography and piezoresponse at the same time. To fully investigate each sample PFM is done in several locations from $\mathrm{PbTiO}_{3}$ that was grown in areas devoid of nanosheets to an area with a dense packing of nanosheets and finally an area on the border between densely packed nanosheets and no nanosheets. This will allow direct comparison between the ferroelectric properties and the size, shape, and surrounding of the ferroelectric nano-objects.

The characterization of the nanosheets and size effects of $\mathrm{PbTiO}_{3}$ grown on said nanosheets is accomplished using the following procedure. First, in section 7.3.1 the size and surface coverage of the $\mathrm{Ca}_{2} \mathrm{Nb}_{3} \mathrm{O}_{10}$ nanosheets deposited on silicon are determined using atomic force microscopy. In the following section, 7.3.2, the growth of the $\mathrm{SrTiO}_{3}$ and $\mathrm{SrRuO}_{3}$ layers examining the difference between the surface morphology measured by AFM of the growth occurring on and off of the underlying nanosheets. Finally, the surface morphology, domain structure, piezoresponse amplitude, and ferroelectric switching properties of the $\mathrm{PbTiO}_{3}$ are characterized for the $\mathrm{PbTiO}_{3}$ deposited off, section 7.3.3, and on top of the $\mathrm{Ca}_{2} \mathrm{Nb}_{3} \mathrm{O}_{10}$ nanosheets, section 7.3.4. The domain structure, piezoresponse, and switching properties were measured using PFM. Finally, in section 7.3.4 the ferroelectric properties from an area containing an abrupt transition from a densely packed nanosheets region to a region without any nanosheets exploring what effect this border has on the ferroelectric response of the $\mathrm{PbTiO}_{3}$.

\subsection{Results}

\subsection{1 $\mathrm{Ca}_{2} \mathrm{Nb}_{3} \mathrm{O}_{10}$ Nanosheets on silicon}

The AFM images of the $\mathrm{Ca}_{2} \mathrm{Nb}_{3} \mathrm{O}_{10}$ nanosheet topography is shown in Figure 7.2. Figure 7.2 a shows a densely packed region of the nanosheets. In the image the size of the nanosheets range from $1.5 \mu \mathrm{m}$ down to $100 \mathrm{~nm}$, the smaller nanosheets are more clearly seen in Figure 7.2i showing an $1800 \mathrm{~nm}$ by $1800 \mathrm{~nm}$ region. Figure $7.2 \mathrm{~b}$ shows a region where the nanosheets are more dispersed. The same range of nanosheet sizes is seen but often the nanosheets are separated from each other with silicon regions in between. Finally Figure 7.2c shows a large region with no nanosheets. Also in this image we see there can be an abrupt 
transition from densely packed regions to no nanosheet regions. Figure $7.2 \mathrm{~d}$ shows a profile over several nanosheets taken from the dashed blue line in Figure 7.2a. The nanosheet surface is very smooth with height differences of only a few picometers. The nanosheets themselves are $\sim 2 \mathrm{~nm}$ high. Based on their crystal structure the $\mathrm{CNO}$ nanosheets are $1.2 \mathrm{~nm}$ thick. The difference between this thickness and the thickness found using AFM is most likely due to the presence of organic molecules left over from the exfoliation from their parent compound.
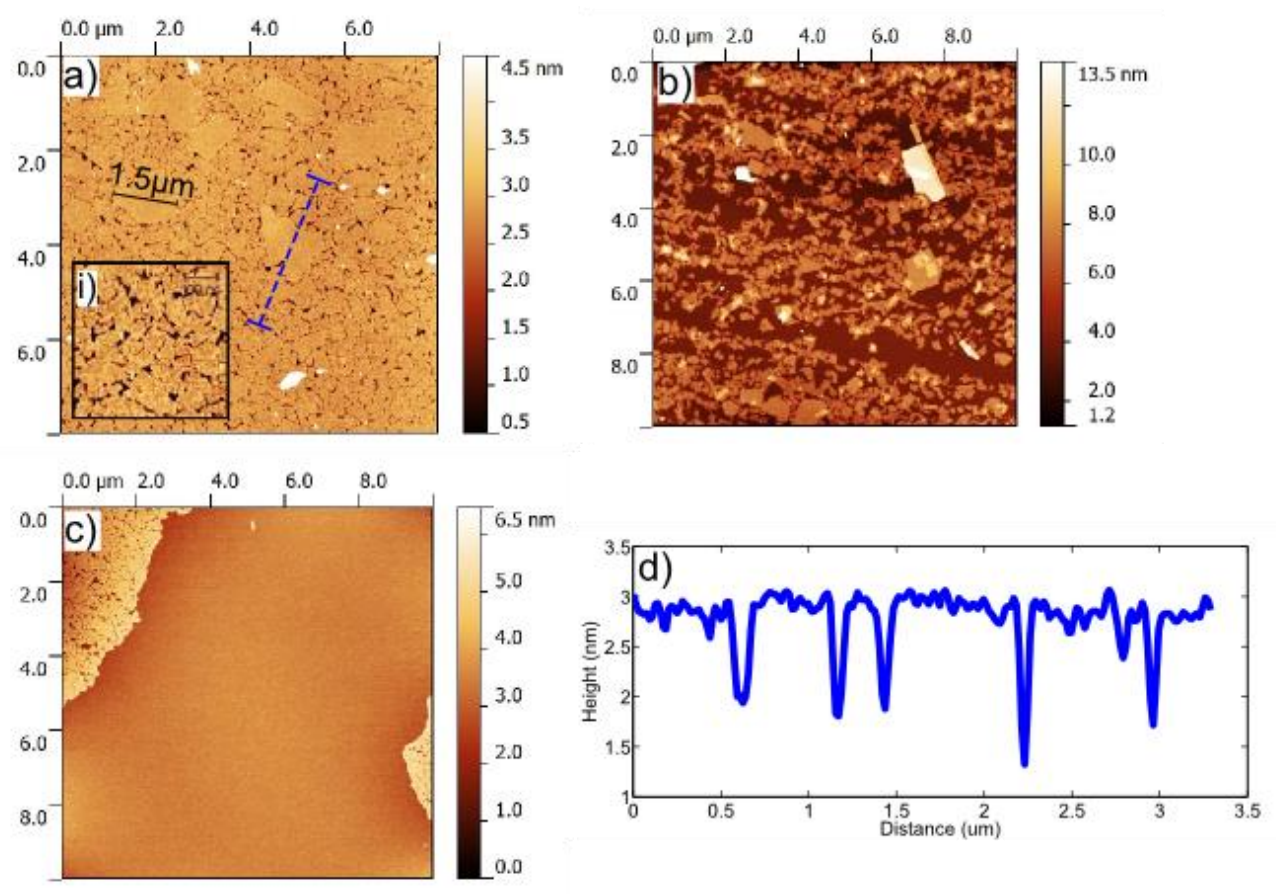

Figure 7.2 CNO nanosheets on Si a) densely packed, b) sparsely packed and c) with no nanosheets d) profile of the densely packed nanosheets.

\subsection{2 $\mathrm{SrRuO}_{3}$ and $\mathrm{SrTiO}_{3}$ growth}

The AFM topography images after the deposition of $\mathrm{SrTiO}_{3}$ and $\mathrm{SrRuO}_{3}$ are shown in Figure 7.3. Figure 7.3a is on a densely packed nanosheet region. The $\mathrm{SrRuO}_{3}$ consists of atomically smooth surfaces with trenches and holes ranging from $0.5 \mathrm{~nm}$ to $2 \mathrm{~nm}$ deep. The RMS of the $\mathrm{SrRuO}_{3}$ film on the densely packed nanosheets is $1.36 \pm 0.320 \mathrm{~nm}$ while the average peak to peak distance for each scan line is $6.95 \pm 2.550 \mathrm{~nm}$. From the surface topography of the $\mathrm{SrRuO}_{3}$ it is now much more difficult to determine the size and shape of the original nanosheets. Only a few clear nanosheet boundaries are visible and are labeled with blue arrows. Figure $7.3 \mathrm{~b}$ shows the topography of a sparsely populated nanosheets region. 
The difference between growth on the nanosheets and the Si is clearly seen by the change in surface topography. Off the nanosheets where the deposited material was grown directly on silicon the film consists of irregularly oriented grains with an RMS of $3.38 \pm 0.345 \mathrm{~nm}$ and an average peak to peak distance of $15.6 \pm 1.99 \mathrm{~nm}$. On the nanosheets the morphology of the $\mathrm{SrRuO}_{3}$ is similar to the densely packed nanosheet regions.
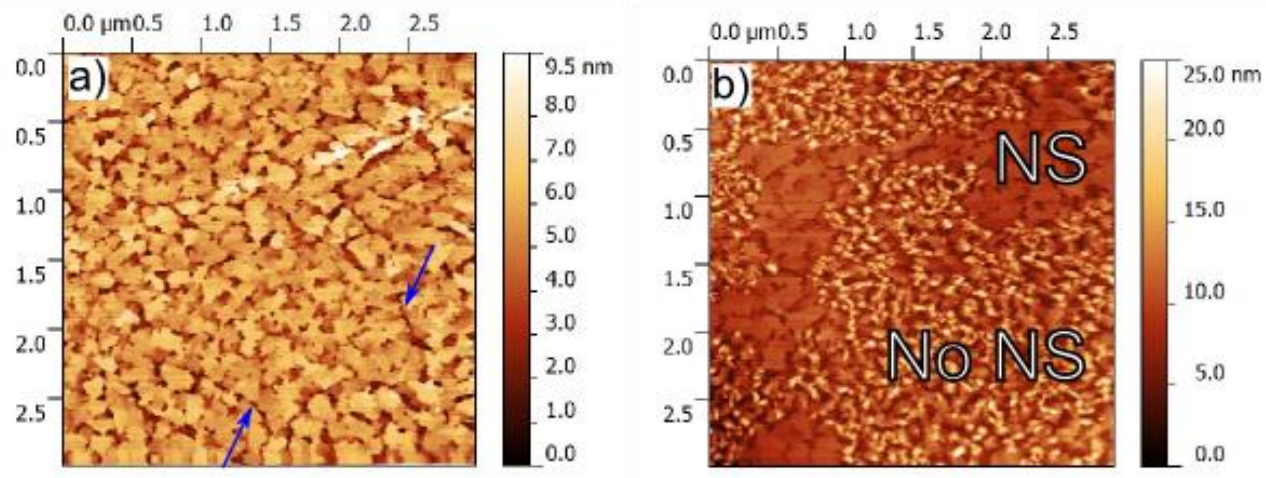

Figure 7.3 $\mathrm{SrTiO}_{3} / \mathrm{SrRuO}_{3}$ thin films on different regions of the sample a) densely packed nanosheets region, b) sparsely packed nanosheets region.

\subsection{3 $\mathrm{PbTiO}_{3}$ off nanosheets}

Atomic force microscopy images showing the surface topography of $\mathrm{PbTiO}_{3}$ grown off of the nanosheets are shown in Figure 7.4 for two different magnifications (Fig. 7.4a $3 \mu \mathrm{m}$ by $3 \mu \mathrm{m}$ area and Fig. $7.4 \mathrm{~b} 1 \mu \mathrm{m}$ by $1 \mu \mathrm{m}$ area). The images show that polycrystalline growth of the $\mathrm{PbTiO}_{3}$ occurred creating a rough surface having an RMS of $3.66 \pm 0.32 \mathrm{~nm}$ and an average peak to peak height of $20.06 \pm 2.11 \mathrm{~nm}$. Closer inspection of the topography in Figure $7.4 \mathrm{~b}$ reveals the surfaces consists of two distinct features: small high grains (green arrow in Figure 7.4b) and slightly larger flat grains (blue arrow in Fig 7.4b). The smaller grains have lateral dimensions ranging from 30-80 $\mathrm{nm}$ with heights up $29 \mathrm{~nm}$, providing the largest contribution to the surface roughness. The flatter grains are about twice as large, with lateral dimensions from 75-200 nm. These flat grains appear to have a smooth surface possibly even atomically smooth. However, their small size makes calculating accurate roughness statistics difficult. These flat grains could be the result of $\mathrm{PbTiO}_{3}$ growth on small sparsely packed nanosheets but this conclusions is ruled out when comparing the nanosheet size and density distribution observed before growth in Figure 7.2. In the sparsely packed nanosheet regions large nanosheets with lateral dimension at least $1 \mu \mathrm{m}$ are observed and such lateral sizes are not present for any $\mathrm{PbTiO}_{3}$ grain. Therefore, the $\mathrm{PbTiO}_{3}$ shown in Figure 7.4 was grown on a region of the substrate that was not covered with any nanosheets. 

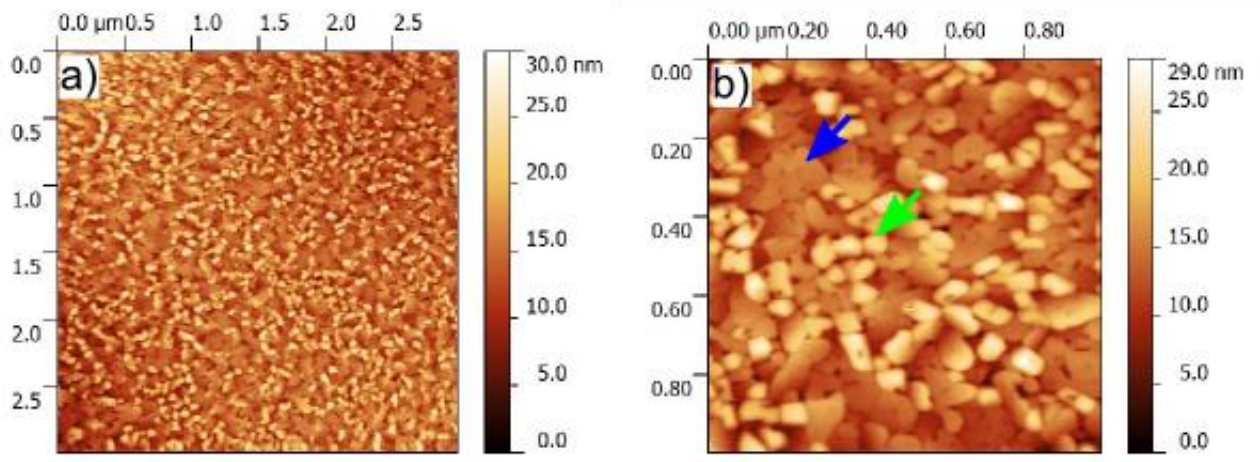

Figure 7.4 AFM images of the surface topography in a a) $3 \mu \mathrm{m}$ by $3 \mu \mathrm{m}$ and b) $1 \mu \mathrm{m}$ by $1 \mu \mathrm{m}$ area after $\mathrm{PbTiO}_{3}$ growth in a region that contained no nanosheets prior to growth. The colored arrows in b) bighlight the two distinct surface features.
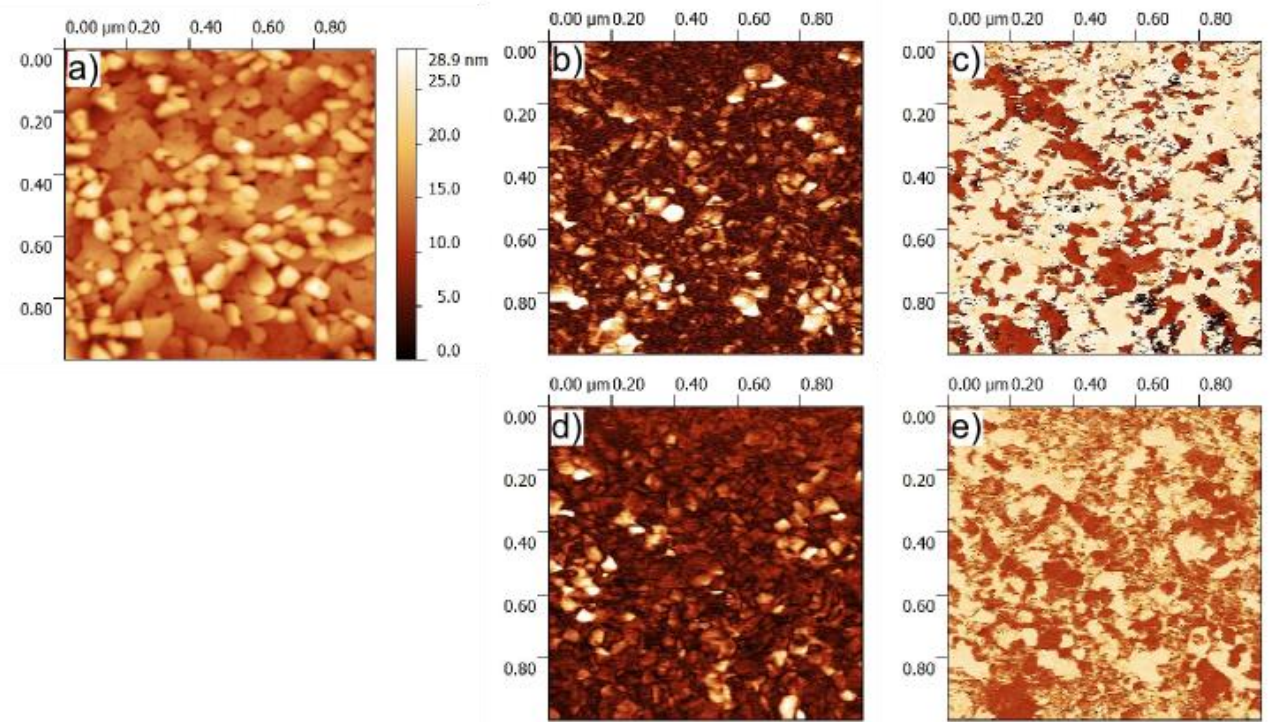

Figure 7.5 a) AFM image of surface topography and corresponding b) out of plane PFM amplitude c)out of plane PFM phase c) in-plane PFM amplitude and d) in-plane PFM phase for $\mathrm{PbTiO}_{3}$ grown in an area that contained no nanosheets prior to growth.

The domain structure of the $\mathrm{PbTiO}_{3}$ film grown off the nanosheets is determined by PFM and shown in Figure 7.5. Figure 7.5a shows an AFM image of the surface topography in 
the region in which the PFM measurements were performed. The out-of plane PFM amplitude and phase response images are shown in Figure $7.5 \mathrm{~b}$ and $7.5 \mathrm{c}$ respectively while the in-plane amplitude and phase response are shown in Figure $7.5 \mathrm{~d}$ and 7.5e. Clear contrast in both out of plane and in-plane response indicates the $\mathrm{PbTiO}_{3}$ has both in-plane and out of plane polarization. The domain morphology, visualized by the PFM phase response, is similar for the out of plane and in-plane directions. In both cases the domains contain large grains of each polarization orientation (up and down for the out of plane and left and right for the inplane). At first glance it seems likely that the domains correspond to the surface morphology and this will be examined more closely in the following paragraph. The amplitude response for the out of plane and in-plane PFM images is also similar to each other. In both cases the piezoresponse amplitude is different for each domain. Some domains exhibit a high piezoresponse (bright areas in Figure 7.5b and 7.5c) in either the out of plane or in plane direction while the other domains the piezoresponse is close to zero. This indicates the polarization occurs in many different orientations supporting the claim the polycrystalline growth has occurred.

To better visualize the relation between the domain structure and surface morphology of the $\mathrm{PbTiO}_{3}$ film grown off the nanosheets $3 \mathrm{D}$ surface contour maps are plotted in Figure 7.6. The two different surface morphologies are highlighted in Figure 7.6a where the small rough grains are colored in red while the large flat grains are colored in blue. The remaining two contour maps are overlaid with out of plane (Figure 7.6b) and in-plane (Figure 7.6c) PFM phase color maps. In all three figures the same surface area is plotted with the only difference being the PFM phase color map that is overlaid on top. Arrows in 7.6a and 7.6b highlight a subsection of the plot where two grains are directly adjacent to one another without any flat region in between. These grains have opposite polarization orientation for both the out of plane and in-plane polarization. The other small rough grains also have a single out of plane and in-plane polarization orientation. Each grain therefore has its own polarization orientation which is due to the polycrystalline growth of the $\mathrm{PbTiO}_{3}$ where the crystal structure of each grain is oriented in a different direction. The flat region in the center of the image does not appear to follow this pattern as the changes in the polarization orientation do not appear to follow any surface features. It is therefore likely each large flat region has the same crystal orientation and these regions and their domain structure behaves similar to a complete film. 


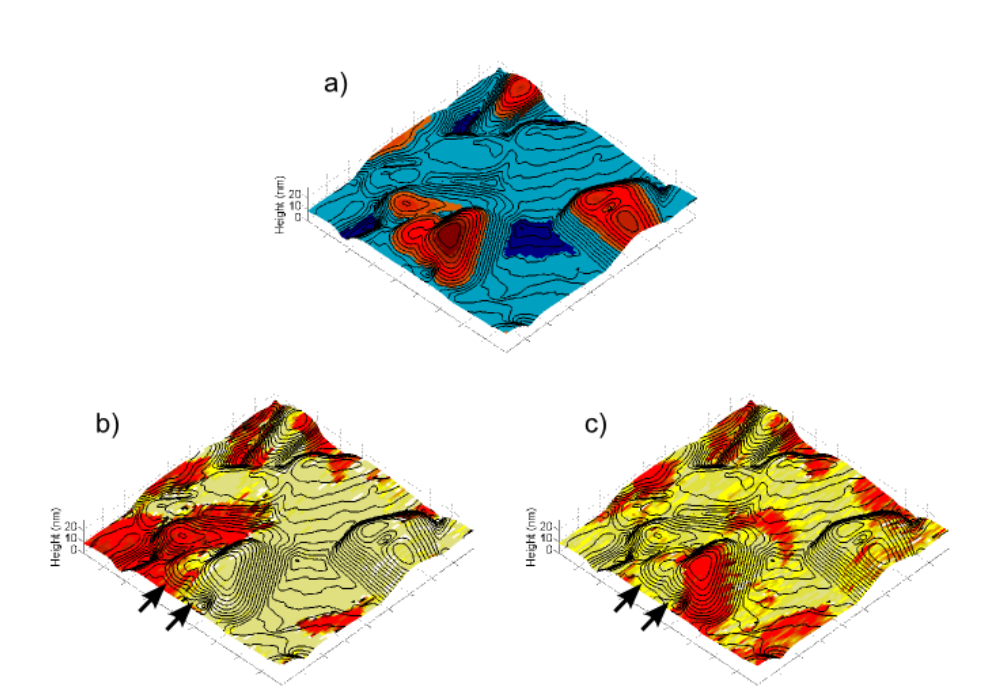

Figure 7.6 Three dimensional surface topography contour maps colored with a) surface topography, bighlighting the two types of surface features (large grains in red and flat regions in blue) b) out of plane PFM phase and b) in-plane PFM phase showing the relationship between the topography and polarization orientation for $\mathrm{PbTiO}_{3}$ grown in an area that contained no nanosheets prior to growth.
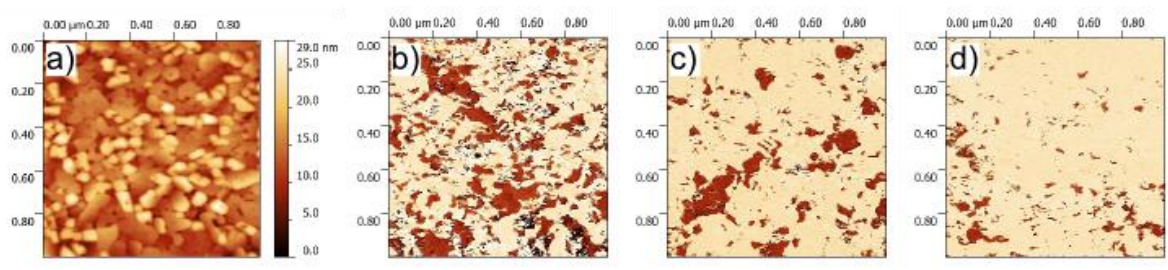

Figure 7.7 a) $A F M$ image of surface topography and corresponding out of plane phase images for b) $0 V_{D C}$ bias c) $0.5 V_{D C}$ bias and d) $1 \mathrm{~V}_{D C}$ bias for $\mathrm{PbTiO}_{3}$ grown in an area that contained no nanosheets prior to growth.

Finally switching experiments were performed by scanning the PFM tip over a region of the film with a constant DC bias to switch the polarization orientation. The resulting PFM phase images of the switching process are shown in Figure 7.7. The regions on and off the nanosheets that are switched are the same as those used in the first set of PFM images, Figure 7.5. The surface topography is shown in Figure 7.7a and the initial PFM out of plane phase image is shown in Figure 7.7b. The PFM phase images after the application of $0.5 \mathrm{~V}$ and $1 \mathrm{~V}$ DC bias are shown in Figure 7.7c and 7.7d respectively. As the applied DC bias increases the polarization orientation of the "dark" regions in the phase images flips to align with the applied 
electric field producing more and more "white" regions in the phase images. The initial film contains $\sim 68 \%$ of the "white" domains and that fraction increases to $82 \%$ and $94 \%$ after $0.5 \mathrm{~V}$ and $1 \mathrm{~V} \mathrm{DC}$ bias respectively. As is clearly seen in Figure $7.7 \mathrm{~d}$ there are a few regions that did not fully switch after the application of $1 \mathrm{~V} \mathrm{DC}$ bias. The remaining dark domains are smaller than their initial size and the further decreasing of their size is expected with the application of high voltages until the film is in a single out of plane domains state.

\subsection{4 $\mathrm{PbTiO}_{3}$ on nanosheets}

The AFM images showing the surface morphology of the $\mathrm{PbTiO}_{3}$ film on an area that had a dense nanosheet coverage is shown in Figure 7.8 for two different magnifications (Fig. $7.8 \mathrm{a} 3 \mu \mathrm{m}$ by $3 \mu \mathrm{m}$ area and Fig. $7.8 \mathrm{~b} 1 \mu \mathrm{m}$ by $1 \mu \mathrm{m}$ area).
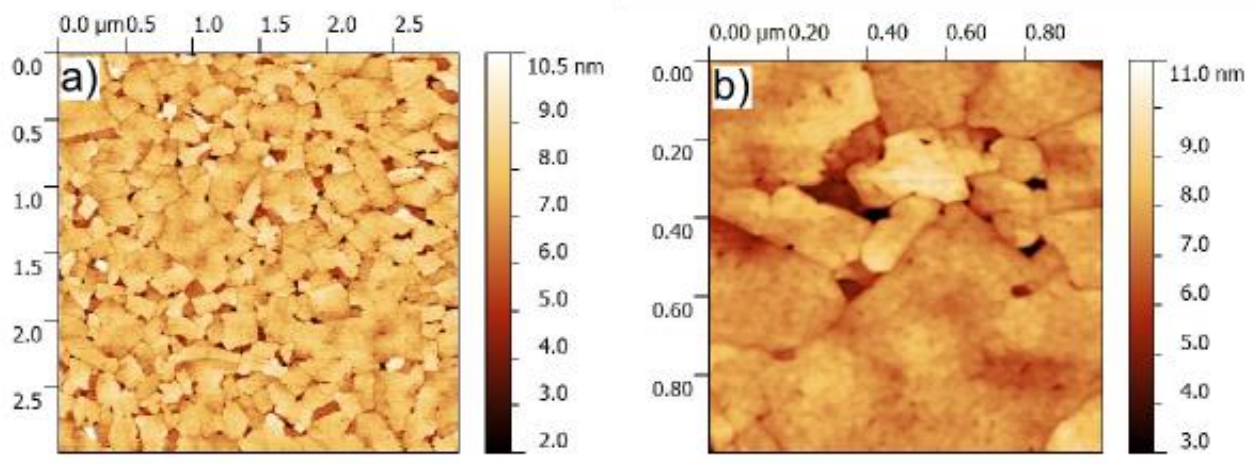

Figure 7.8 AFM images of the surface topography in a a) $3 \mu \mathrm{m}$ by $3 \mu \mathrm{m}$ and b) $1 \mu \mathrm{m}$ by $1 \mu \mathrm{m}$ area after $\mathrm{PbTiO}_{3}$ growth in a region that contained a dense packing of nanosheets prior to growth.

The surface consists of flat smooth areas with lateral dimensions ranging from $1.5 \mu \mathrm{m}$ down to $100 \mathrm{~nm}$. The surface roughness of the Figure $7.8 \mathrm{a}$ is $0.989 \pm 1.8 \mathrm{~nm}$ with an average peak to peak height of $6.57 \pm 1.59 \mathrm{~nm}$. The size range and shapes of the $\mathrm{PbTiO}_{3}$ corresponds with the initial nanosheet dimensions. As a result, the $\mathrm{PbTiO}_{3}$ objects observed in Figure 7.8 are interpreted as being individual nanosheets covered with a $\mathrm{PbTiO}_{3}$ film. The atomically smooth growth of the $\mathrm{PbTiO}_{3}$ on each nanosheet strongly indicates that on each nanosheet the film is single crystalline. The dense packing of the $\mathrm{PbTiO}_{3}$ covered nanosheets is more easily observed in Figure 7.8b. There are a few regions in the center of the image where small trenches are visible where the $\mathrm{PbTiO}_{3}$ covered nanosheets did not conform to one another but for the most part the nanosheets edges are packed directly against each other. The edges between adjacent nanosheets are often observable by small height differences of 1-3 nm. However, based on the surface morphology alone it is not possible to determine if the $\mathrm{PbTiO}_{3}$ films on adjacent closely packed nanosheets are completely separated from each other. 
The out of plane and in-plane PFM images showing the domain structure of the $\mathrm{PbTiO}_{3}$ on densely packed nanosheets is shown in Figure 7.9.
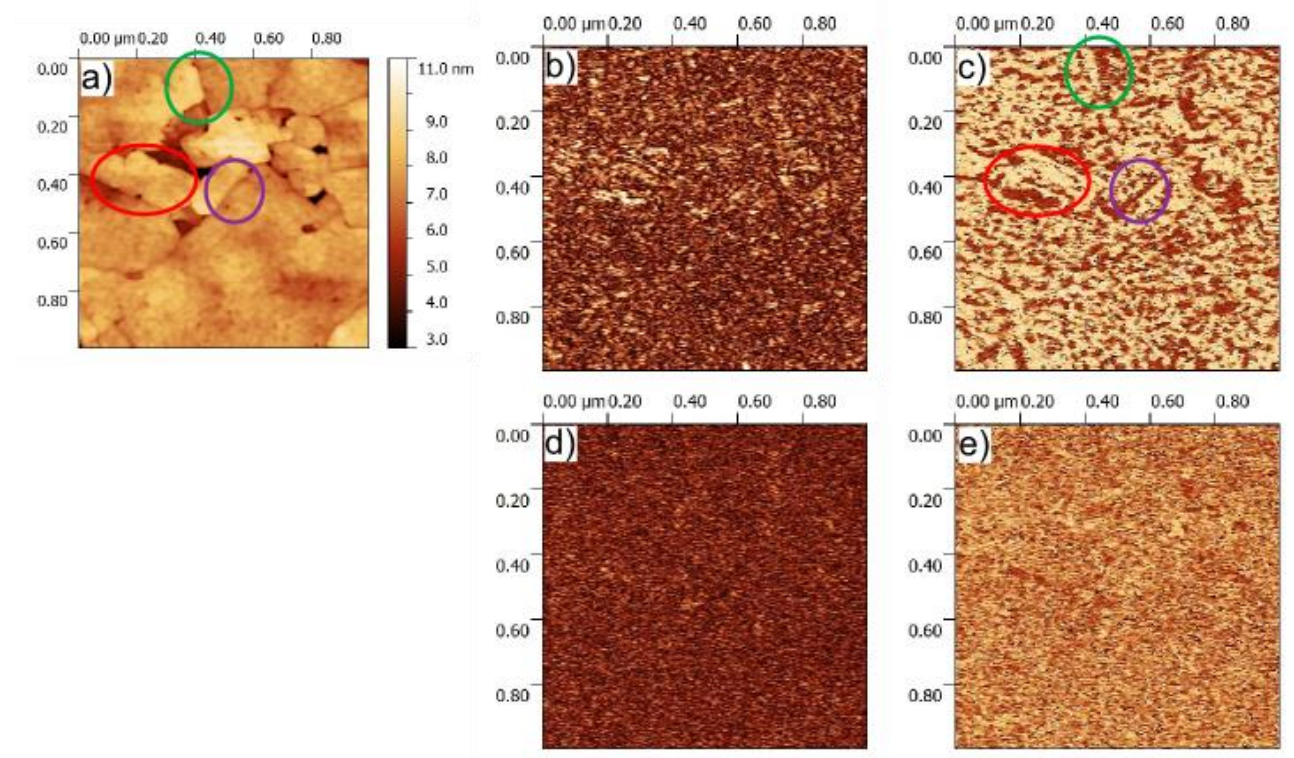

Figure 7.9 a) AFM image of surface topography and corresponding b) out of plane PFM amplitude c)out of plane PFM phase c) in-plane PFM amplitude and d) in-plane PFM phase for $\mathrm{PbTiO}_{3}$ grown in an area that contained a dense packing of nanosheets prior to growth. Three different colored circles are used to bighlight the relationship between the nanosheet edges and straight domains walls

The surface topography corresponding to the area investigated by PFM is shown in Figure 7.9a. In this area there is one large nanosheet with lateral dimension of $\sim 1 \mu \mathrm{m}$ and several smaller nanosheets with sizes down to $150 \mathrm{~nm}$. The out of plane amplitude and phase response is shown in Figures $7.9 \mathrm{~b}$ and $7.9 \mathrm{c}$ respectively while the in-plane amplitude and phase response is shown in Figures 7.9 d and 7.9e respectively. The domain structure observed in this region is completely different from the $\mathrm{PbTiO}_{3}$ film off of the nanosheets. In the out of plane piezoresponse two different types of domains are observed. First, at some of the $\mathrm{PbTiO}_{3}$ covered nanosheet boundaries long straight domains are formed. The nanosheet edges and the corresponding long straight domains are highlighted by the colored circles in the Figure 9a and c. The second much more prevalent domain morphology is irregular rounded domains. These domains do not have a uniform size or shape but are characterized by squiggly domain walls. These domains are small, having an average size of $\sim 30 \mathrm{~nm}$. Furthermore, these is no difference in the domain morphology of these small squiggly domains based on the size of the $\mathrm{PbTiO}_{3}$ covered nanosheet or the location of the domains within a single nanosheet: either in the center or towards the edge. The out of plane amplitude response for both types of 
domains remains constant over the entire scan region. The only contrast visible in the out of plane amplitude image is where the response goes to zero at the domain walls. Finally for the in-plane PFM measurements no signal was observed. The contrast in the amplitude and phase images is random noise and no in-plane polarization is present in the $\mathrm{PbTiO}_{3}$ on the nanosheets. As a result the film on the nanosheets contains only $180^{\circ}$ domains.

To look more closely at the relationship between the domain wall edges and the long straight domains that sometimes form along the edges 3D surface topography contour maps overlaid with the out of plane PFM phase color map is shown in Figure 7.10 for two different location in Figure 7.9a.
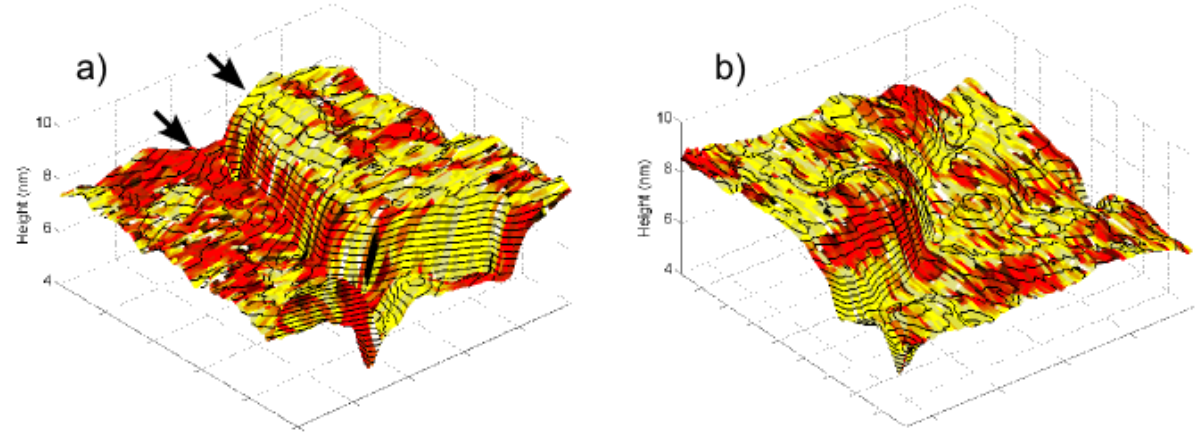

Figure 7.10 Three dimensional surface topography contour maps colored with out of plane PFM phase for two different locations of $\mathrm{PbTiO}_{3}$ grown in areas that contained a dense packing of nanosheets prior to growth showing a) a change in out of plane polarization with a height difference between two nanosheets and b) no change in out of plane polarization with height difference between two nanosheets

Figure 7.10a corresponds with the area highlighted by the green circle in Figure 7.9a and shows an area of 193 by $193 \mathrm{~nm}$. Here the nanosheet edge is distinguished by a step in the surface topography of $\sim 2 \mathrm{~nm}$. The PFM phase contrast on either side of this edge is highlighted with the two arrows. The $\mathrm{PbTiO}_{3}$ covered nanosheet edge on the lower side contains only "dark" contrast. The polarization orientation then changes up the step to where it is flipped to the "white" contrast on the higher $\mathrm{PbTiO}_{3}$ covered nanosheet. It is tempting to conclude that $\mathrm{PbTiO}_{3}$ covered nanosheet edges that are accompanied by a change in height will always result in the formation of long straight domains along the nanosheets edges, however, this is not the case. Figure 7.10b shows a 193 by $193 \mathrm{~nm}$ area corresponding to the lower right corner in Figure 7.9a. Here a clear step is observed between the two $\mathrm{PbTiO}_{3}$ covered nanosheets that meet in this location. Unlike the edge in Figure 7.10a, the polarization 
orientation does change with the surface morphology. In this region the more common smaller squiggly domains run across the edge without any change in the domain morphology.
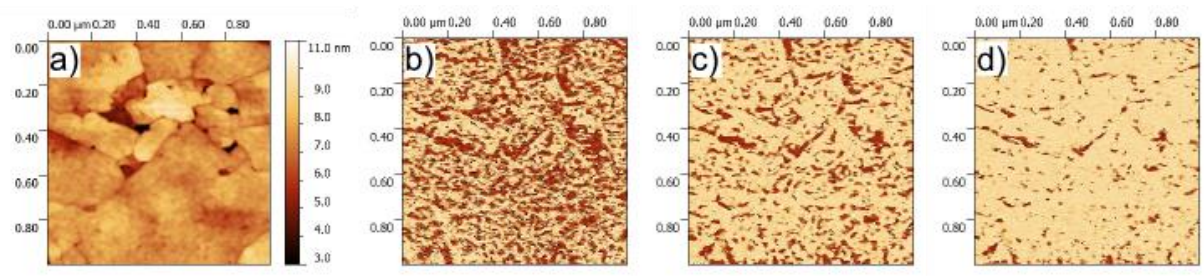

Figure 7.11 a) $A F M$ image of surface topography and corresponding out of plane phase images for b) $0 V_{D C}$ bias c) $0.5 V_{D C}$ bias and d) $1 V_{D C}$ bias for $\mathrm{PbTiO}_{3}$ grown in an area that contained a dense packing of nanosheets prior to growth.

The same switching experiments using a DC bias applied to the tip was performed and the results are shown in Figure 7.11. Figure 7.11a shows the surface topography where the switching experiment took place. Figure $7.11 \mathrm{~b}$ is the out of plane PFM phase image of the initial domain structure without any applied bias. The PFM phase image after the application $0.5 \mathrm{~V}$ and $1 \mathrm{~V} \mathrm{DC}$ bias is shown in Figure $7.11 \mathrm{c}$ and $7.11 \mathrm{~d}$ respectively. The starting domain pattern consisted of $63 \%$ "white" domains. This domain fraction increased with applied DC bias to $82 \%$ and $94 \%$ with $0.5 \mathrm{~V}$ and $1 \mathrm{~V} \mathrm{DC}$ bias respectively. These domain fractions are very similar to the results obtain from switching the $\mathrm{PbTiO}_{3}$ film off the nanosheets, Figure 7.7, which indicate that the physics of the switching process are not affected by the underlying nanosheets. Of the remaining unswitched polarization, the "dark" domains, after the application of $1 \mathrm{~V}$ DC bias most belong to the long straight domain along the $\mathrm{PbTiO}_{3}$ covered nanosheet edges. These domains have undergone a reduction in their width but maintain their initial length.

The PFM investigation in Figure 7.9 was done on a dense packing of the $\mathrm{PbTiO}_{3}$ covered nanosheets. The dense packing might be influencing the domain structure and piezoresponse by the film being mechanically impeded by material from the adjacent $\mathrm{PbTiO}_{3}$ covered nanosheets. Ideally we would like to measure the piezoresponse from isolated $\mathrm{PbTiO}_{3}$ covered nanosheets in a sparsely populated region where the influence of any surrounding $\mathrm{PbTiO}_{3}$ covered nanosheets is completely eliminated. However, in the sparsely packed nanosheet regions it became difficult to identify the boundaries of the $\mathrm{PbTiO}_{3}$ grown on the nanosheets and off making precise correlation with the underlying nanosheets difficult. Therefore, to search for an increase of piezoresponse a border region between a densely packed and no nanosheet region is examined and shown in Figure 7.12. 

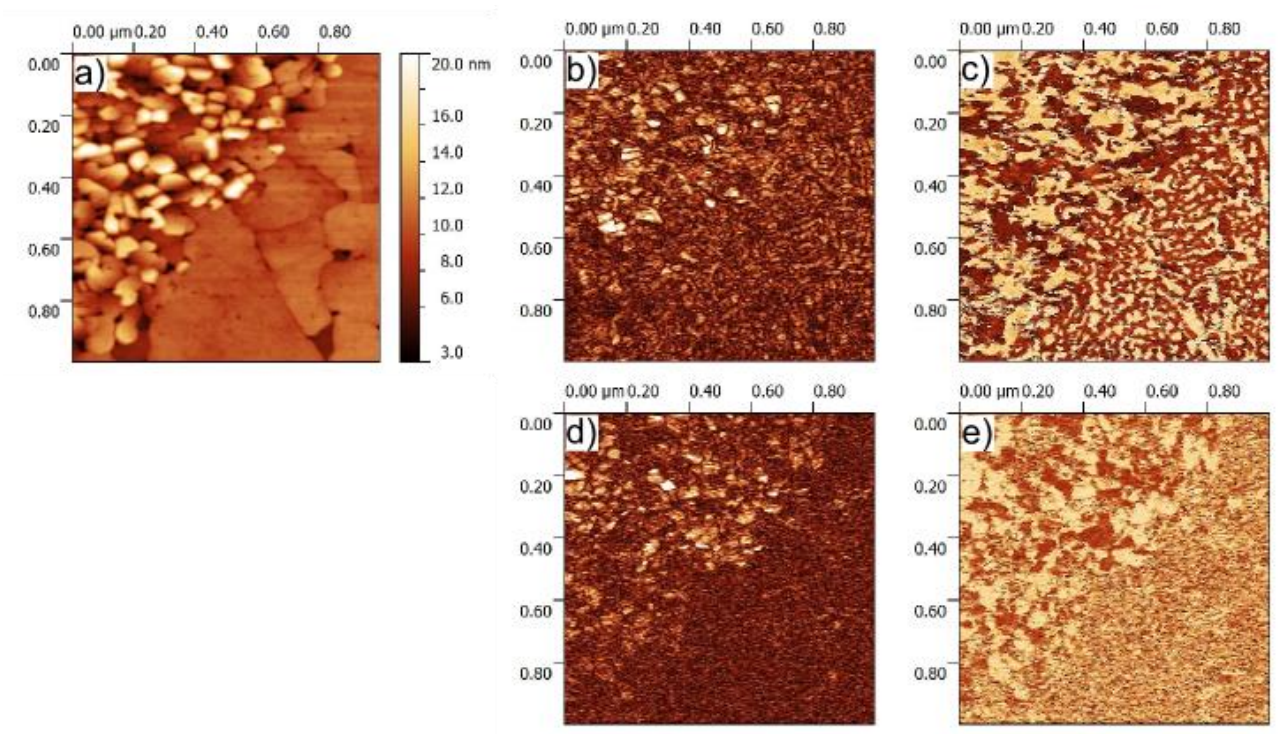

Figure 7.12 a) AFM image of surface topography and corresponding b) out of plane PFM amplitude c)out of plane PFM phase c) in-plane PFM amplitude and d) in-plane PFM phase for $\mathrm{PbTiO}_{3}$ grown in an area that contained no nanosheets prior to growth.

At this interface there should be less mechanical clamping between the single crystalline $\mathrm{PbTiO}_{3}$ on the nanosheets and the polycrystalline $\mathrm{PbTiO}_{3}$ off the nanosheets. The difference between the nanosheet and no nanosheet regions is clearly seen in the AFM images of the surface topography, Figure 7.12a. The upper left half of the image contains the rough grains consistent with the $\mathrm{PbTiO}_{3}$ film grown off the nanosheets as also shown in Figure 7.4. The bottom right half of Figure 7.12a comprises the $\mathrm{PbTiO}_{3}$ covered nanosheets. Several nanosheets with lateral dimensions from $500 \mathrm{~nm}$ down to $100 \mathrm{~nm}$ are visible. The domain structure and piezoresponse seen in the out of plane and in-plane PFM images, Figures 7.12 b$\mathrm{d}$, show the same behavior in the previous measurements for the $\mathrm{PbTiO}_{3}$ grown on or off the nanosheets matching the domain patterns observed in Figures 7.5 and 7.9 for the off and on nanosheet regions respectively. No increase or decrease in the piezoresponse amplitude or change in the domain structure is observed at the interface between the nanosheets and no nanosheets regions.

To quantify how the piezoresponse changes from a non-nanosheet region to the densely packed region line profiles of the surface topography and in-plane PFM phase are plotted in Figure 7.13 across the boundary between these two regions. The in-plane PFM phase was chosen because this signal goes from a definite value in the area with $\mathrm{PbTiO}_{3}$ off the nanosheets, where there is clear in-plane PFM response, to just noise on the $\mathrm{PbTiO}_{3}$ covering the nanosheets, where there is no in-plane piezoresponse. The border between these two 
regions was identified from the surface topography profile and is emphasized by the vertical black dotted line in Figure 7.13. To the left of this line in the dark grey area two large grains are visible in the surface topography with heights of just over 20 and $10 \mathrm{~nm}$. To the right of the black line the surface topography becomes flat indicating the change to a $\mathrm{PbTiO}_{3}$ covered nanosheet. The in-plane PFM phase response starts to decrease immediately at the change between on and off the nanosheets and reaches zero $9 \mathrm{~nm}$ onto the $\mathrm{PbTiO}_{3}$ covered nanosheets.

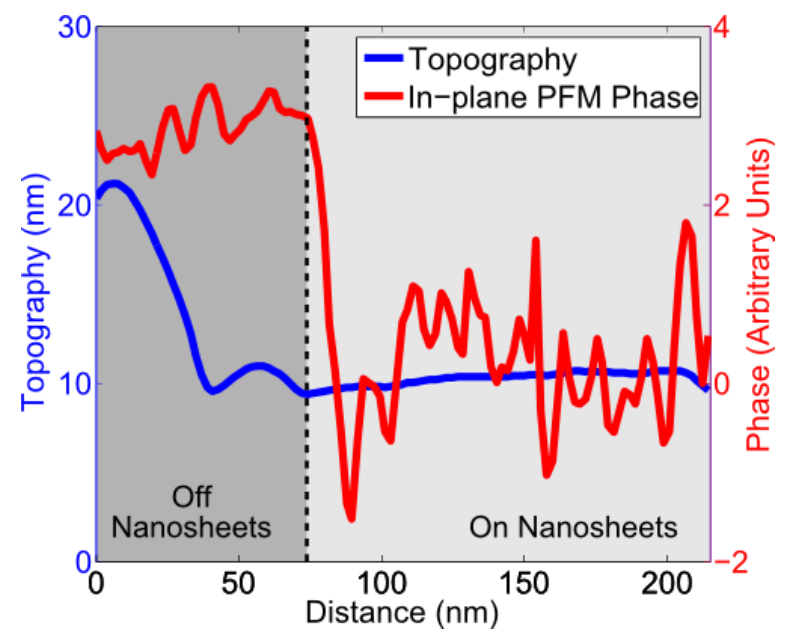

Figure 7.13 Schematic of the samples used to grow $\mathrm{PbTiO}_{3}$ nanoislands showing the multitude of nanosheets sizes and densities in a single sample.

\subsection{Discussion}

The results obtained from thin films of $\mathrm{PbTiO}_{3}$ on $\mathrm{CNO}$ covered $\mathrm{Si}$ provided information on the growth and domain structure of $\mathrm{PbTiO}_{3}$. First and foremost there is a clear difference in the morphology of the $\mathrm{PbTiO}_{3}$ on and off the nanosheets. Off the nanosheets, where the $\mathrm{PbTiO}_{3}$ grows on the silicon, polycrystalline growth occurs as evidenced by the distinct randomly oriented grains observed in the AFM surface topography images. On the nanosheets the $\mathrm{PbTiO}_{3}$ growth is much smoother. No clear steps or other features are observed on the $\mathrm{PbTiO}_{3}$ surface within a single nanosheet indicating that $\mathrm{PbTiO}_{3}$ is single crystalline on each nanosheet. The lack of any in-plane PFM signal on the nanosheets indicates that the $\mathrm{PbTiO}_{3}$ is completely $c$ oriented where the long axis of the tetragonal $\mathrm{PbTiO}_{3}$ unit cell is perpendicular to the film surface producing only $180^{\circ}$ domains. The difference in growth between the single crystalline $\mathrm{PbTiO}_{3}$ on the nanosheets and the polycrystalline $\mathrm{PbTiO}_{3}$ off the nanosheets is important for the study of size effects of the domains in the $\mathrm{PbTiO}_{3}$. This makes the identification of the initial surface the $\mathrm{PbTiO}_{3}$ was grown on 
(nanosheets or silicon) and individual nanosheets easy to identify, allowing precise correlation between the properties of the film and the underlying surface.

On the nanosheets the domain structure of the $\mathrm{PbTiO}_{3}$ is much different from complete epitaxial thin films and the domain structure predicted from various theoretical models for $c$-axis oriented $\mathrm{PbTiO}_{3}$ and what was observed previous experimental findings, Figure 4.3. Most striking is the lack of a periodic striped domain pattern for the $180^{\circ}$ domains on the nanosheets. Instead the domain structure consists of small irregularly shaped domains with an average size of approximately $30 \mathrm{~nm}$, determined from Fourier transforms of the PFM images. This result is not expected since domain walls cost energy to produce and a stripe domain pattern with straight domain walls will minimize the total length of the domain walls in the film. The lack of any in-plane domains means that the cause of the domain pattern observed is uniquely due to the electrical boundary conditions in this system. This unusual domain pattern could be a result of the lateral size effect of the $\mathrm{PbTiO}_{3}$ on the nanosheets. The depolarizing field resulting from bound surface charges from a continuous ferroelectric film is oriented perpendicular to the film surface and is pointed in the opposite direction of the polarization. In the case of a continuous film, effects from the film edges where the electric field deviates from this strictly perpendicular field are not expected to have any effect since this constitutes a minute portion of the overall film. With the limited lateral dimensions of the nanosheets this deviation in the direction of the depolarizing field will now occupy a large area for each $\mathrm{PbTiO}_{3}$ covered nanosheet and could result in domain structures not predicted in Kittel-like models for ferroelectric domains. One problem with this picture is the domain size and domain pattern did not change with different nanosheets size from $\mathrm{PbTiO}_{3}$ covered nanosheets ranging from $1.5 \mu \mathrm{m}$ to $100 \mathrm{~nm}$. Furthermore, within a single nanosheet the size and pattern of the domains is always the same in the center of the nanosheet as on the edges, except for the long straight domains occurring at some nanosheet edges. One could argue that the lack of deviation of the domain pattern at the edges of the nanosheet could be due to the close packed structure of the nanosheets in the region of the film explored where the $\mathrm{PbTiO}_{3}$ could have formed more of a complete film. However, the same phenomenon is observed at the region of the film where the closed packed nanosheets bordered with a $\mathrm{PbTiO}_{3}$ grown on silicon. At the border of these two regions the single crystalline film on the nanosheets should be much more separate from the polycrystalline region. However, no change to the domain structure is observed in the $\mathrm{PbTiO}_{3}$ at this location. In a few places the straight domains are observed at the edges of the nanosheets, deviating from the overall circular domain pattern. The switching experiments showed that these regions were the last to switch remaining in their original polarization state at voltages above the rest of the film.

A common observation in many of the lateral size effect studies cited in the introduction to this chapter was the occurrence of increased piezoresponse amplitude at the edges of the nanoscale ferroelectrics. In this study no enhancement of the piezoresponse was observed at any of the $\mathrm{PbTiO}_{3}$ covered nanosheet edges. This includes regions where the 
nanosheets were densely packed and regions where the $\mathrm{PbTiO}_{3}$ covered nanosheet were bordering $\mathrm{PbTiO}_{3}$ grown where no nanosheets were present. The reason for the increase in piezoresponse in other studies is the relaxation of epitaxial strain as the edge of the nanoscale ferroelectric experiences less clamping. This relaxation in the strain is often seen as a reduction in the fraction of $a$ domains in the ferroelectric. To understand why no increase in the piezoresponse was observed for the $\mathrm{PbTiO}_{3}$ covered nanosheets several factors need to be considered including the misfit strain between the $\mathrm{PbTiO}_{3}$ and the nanosheets, the difference in thermal expansion between the $\mathrm{PbTiO}_{3}$ and the silicon substrate, and the amount of clamping between the nanosheets and the silicon substrate. The least understood of these factors is the amount of clamping between the nanosheets and the silicon. If the nanosheets are weakly clamped to the silicon then are free to deform with applied stress. In this case the lattice mismatch between the nanosheets and the $\mathrm{PbTiO}_{3}$ will be relaxed through a deformation of the nanosheets since the nanosheets consist of a single layer of while the $\mathrm{PbTiO}_{3}$ film was $30 \mathrm{~nm}$ thick. Therefore, the $\mathrm{PbTiO}_{3}$ film is, in effect, unstrained and as a result there can be no increase of the piezoresponse due a reduction of $a$ domains from strain relaxation at the edges of the nanosheet as there is no strain to relax.

The other extreme is that the nanosheets are completely clamped to the silicon substrate and are able to deform only with a change in the silicon due to thermal expansion. The misfit strain between the nanosheets and $\mathrm{PbTiO}_{3}$ results in a compressive strain of $-1.15 \%$ at room temperature. At the deposition temperature of $600^{\circ} \mathrm{C}$ this compressive strain would increase as the thermal expansion coefficient of $\mathrm{PbTiO}_{3}$ is larger than silicon by a factor of $\sim 4.5$. The compressive strain experienced by the film at the transition temperature during cool down after growth would favor the formation of purely $c$ axis oriented $\mathrm{PbTiO}_{3}$ on the nanosheets with no $a$ domains present. No enhancement of the piezoresponse occurs since there are no $a$ domains present. In reality the clamping of the nanosheets will be somewhere between these two extremes. In any case what is clear from the PFM measurements is that no in-plane polarization is present in any of the $\mathrm{PbTiO}_{3}$ covered nanosheets, large or small. As a result the enhancement of piezoelectricity due to a reduction of $a$ domains occurring due to reduced clamping from the substrate simply cannot occur as no $a$ domains are present.

Even in the absence of $a$ domains clamping of a ferroelectric film reduces its piezoresponse as the material is not free to fully deform with the applied field. If the $\mathrm{PbTiO}_{3}$ covered nanosheets are compressively strained, which is likely due to only $180^{\circ}$ domains being present, then relaxation is expected to take place at the around the edges resulting in the an increase of the piezoresponse. The reason this is not observed is attributed to the presence of the neighboring $\mathrm{PbTiO}_{3}$. In the densely packed nanosheet regions the close packing could be restricting the ability of the $\mathrm{PbTiO}_{3}$ covered nanosheets to relax their compressive strain. A boarder region between dense nanosheet packing and a no nanosheet region was investigated with the hope that the single crystalline $\mathrm{PbTiO}_{3}$ on the nanosheets would not be so mechanically restricted by the neighboring polycrystalline $\mathrm{PbTiO}_{3}$. However, no gap between 
polycrystalline and single crystalline $\mathrm{PbTiO}_{3}$ was present and the lack of any increase in the piezoresponse from strain relaxation is again attributed to the $\mathrm{PbTiO}_{3}$ being restricted by the surrounding material. A solution to this problem would be to selectively remove the polycrystalline $\mathrm{PbTiO}_{3}$ grown off the nanosheets. However, there is no possibility to etch only the polycrystalline $\mathrm{PbTiO}_{3}$ while leaving the $\mathrm{PbTiO}_{3}$ on the nanosheets undamaged. Another possibility has recently been developed by using a sacrificial substrate which is etched away creating a suspension of the $\mathrm{PbTiO}_{3}$ covered nanosheets in solution which could then be redeposited on a new substrate allowing completely separated $\mathrm{PbTiO}_{3}$ covered nanosheets to be characterized.

\subsection{Conclusions}

In this chapter a $\mathrm{PbTiO}_{3}$ thin film was grown on $\mathrm{CNO}$ nanosheet coated silicon to examine the lateral size effects on the ferroelectric domains and the piezoresponse. The laterals sizes of the individual nanosheets range from $1.5 \mu \mathrm{m}$ to $100 \mathrm{~nm}$ providing a system in which the lateral size effects can be effectively studied in one sample. The difference in growth between $\mathrm{PbTiO}_{3}$ on and off the nanosheets and the high quality growth of the $\mathrm{PbTiO}_{3}$ on each separate nanosheet allowed for easy identification of the underlying surface through the topography of the ferroelectric film surface.

On the $\mathrm{PbTiO}_{3}$ covered nanosheets only $180^{\circ}$ domains are observed and unlike ferroelectric films without any lateral constraints the domain structure of the $\mathrm{PbTiO}_{3}$ on the nanosheets consisted of small irregular domains of $\sim 30 \mathrm{~nm}$. In some cases long straight domains are formed at the edges of the $\mathrm{PbTiO}_{3}$ covered nanosheets. In addition no change in the domain size or pattern occurred based on the size of the $\mathrm{PbTiO}_{3}$ covered nanosheets or location within a single $\mathrm{PbTiO}_{3}$ covered nanosheets (with the exception of the few straight domains). The lack of any in-plane domains points to electrostatic effects being the cause of these domain and these effects do not change over the length scales of $1.5 \mu \mathrm{m}$ to $100 \mathrm{~nm}$ studied using these nanosheet sizes.

Finally, no enhancement of the piezoresponse is observed in any region of the ferroelectric covered nanosheets. The response amplitude is uniform within each $\mathrm{PbTiO}_{3}$ covered nanosheets, at the edges between two $\mathrm{PbTiO}_{3}$ covered nanosheets and on the border between a densely covered nanosheet region and $\mathrm{PbTiO}_{3}$ grown on silicon region. The exact strain state of the $\mathrm{PbTiO}_{3}$ on the nanosheet will depend on the degree of clamping between the nanosheets and the silicon. If there is no clamping then any strain in the $\mathrm{PbTiO}_{3}$ will be imparted to the nanosheet. As a result, the $\mathrm{PbTiO}_{3}$ will be strain free and there is no strain to be relaxed at the edges of the nanosheet. In the opposite case where the $\mathrm{PbTiO}_{3}$ is strongly clamped a $c$ axis oriented film is expected and enhancement of the piezoresponse due to a reduction of $a$ domains cannot occur. In either case, experimentally no in-plane piezoresponse 
is observed in the PFM measurements for the $\mathrm{PbTiO}_{3}$ covered nanosheets meaning enhancement of the piezoresponse due to a reduction of $a$ domains is not possible in this system. Another reason for the lack of any observed increase of the piezoresponse is due to the close packed nature of the $\mathrm{PbTiO}_{3}$ both on and off the nanosheets which mechanically restricts the $\mathrm{PbTiO}_{3}$ covered nanosheets from relaxing. 


\section{References}

[1] C. S. Ganpule, A. Stanishevsky, Q. Su, S. Aggarwal, J. Melngailis, E. Williams, et al., "Scaling of ferroelectric properties in thin films," Applied Physics Letters, vol. 75, pp. 409-411, Jul 191999.

[2] C. S. Ganpule, A. Stanishevsky, S. Aggarwal, J. Melngailis, E. Williams, R. Ramesh, et al., "Scaling of ferroelectric and piezoelectric properties in $\mathrm{Pt} / \mathrm{SrBi} 2 \mathrm{Ta} 2 \mathrm{O} 9 / \mathrm{Pt}$ thin films," Applied Physics Letters, vol. 75, pp. 3874-3876, Dec 131999.

[3] V. Nagarajan, A. Roytburd, A. Stanishevsky, S. Prasertchoung, T. Zhao, L. Chen, et al., "Dynamics of ferroelastic domains in ferroelectric thin films," Nature Materials, vol. 2, pp. 43-47, Jan 2003.

[4] S. Buhlmann, B. Dwir, J. Baborowski, and P. Muralt, "Size effect in mesoscopic epitaxial ferroelectric structures: Increase of piezoelectric response with decreasing feature size," Applied Physics Letters, vol. 80, pp. 3195-3197, Apr 292002.

[5] A. Roelofs, T. Schneller, K. Szot, and R. Waser, "Towards the limit of ferroelectric nanosized grains," Nanotechnology, vol. 14, pp. 250-253, Feb 2003.

[6] H. Fujisawa, K. Morimoto, M. Shimizu, and H. Niu, "Observations of island structures at the initial growth stage of PbZrxTi1-xO3 thin films prepared by metalorganic chemical vapor deposition," Japanese Journal of Applied Physics Part 1 Regular Papers Short Notes \& Review Papers, vol. 39, pp. 5446-5450, Sep 2000.

[7] H. Han, K. Lee, W. Lee, M. Alexe, D. Hesse, and S. Baik, "Fabrication of epitaxial nanostructured ferroelectrics and investigation of their domain structures," Journal of Materials Science, vol. 44, pp. 5167-5181, Oct 2009.

[8] M. Nijland, S. Kumar, R. Lubbers, D. H. A. Blank, G. Rijnders, G. Koster, et al., "Local Control over Nucleation of Epitaxial Thin Films by Seed Layers of Inorganic Nanosheets," ACS Applied Materials \& Interfaces, 2014. 


\section{Summary}

Ferroelectrics have become an important class of materials due to their wide range of attractive properties. From high $\mathrm{K}$ dielectrics for capacitors, to pyroelectric infrared detectors, to piezoelectric actuators and sensors in MEMs devices and even non-volatile computer memories ferroelectric materials are used in many different technological domains. Integration of ferroelectrics into electronic devices produces a drive to make the ferroelectric components smaller and smaller to keep up with the increased demand for portable electronics with greater functionality. However, the attractive properties of ferroelectrics are known to degrade as the size of the ferroelectric material in one or more dimensions enters the nanoscale. Therefore, understanding how ferroelectric materials behave when fabricated into nanoscale objects and how their properties can be maintained on this length scale is an active area of current research.

Many studies of size effects in ferroelectrics use top-down patterning techniques to define nanoscale ferroelectric objects from ferroelectric films. In many cases Focused Ion Beam (FIB) and electron beam lithography are used to pattern ferroelectric objects with dimensions down to less than $100 \mathrm{~nm}$. However, top-down techniques introduce damage to the ferroelectric such as ion implantation and resputtering. The sensitive relation between the crystal structure of a ferroelectric and its properties means that such damage can produce extrinsic effects, masking the true influence of the size effect on the ferroelectric properties. Bottom-up approaches do not suffer from the drawbacks of the top-down techniques as they utilize naturally occurring self-assembly processes to create nanosized objects. However, control over the size and shape and position of the resulting nanoscale features is an issue.

In this thesis the size effects of ferroelectrics is explored in a novel way by modulating the boundary conditions, under which the ferroelectric is subjected, on the nanoscale. The act of patterning a ferroelectric into nanoscale objects changes the boundary conditions resulting in size effects. Therefore, an alternative method of studying nanoscale ferroelectrics is to investigate the response of a ferroelectric film to nanoscale changes in the boundary conditions under which the ferroelectric is subjected. This is accomplished by engineering on the nanoscale the bottom interface of the ferroelectric film. This approach has the benefit of eliminating post processing of the ferroelectric material which removes extrinsic effects from the investigation of the ferroelectric properties. In addition, by using selfassembly techniques to engineer the bottom interface the entire sample is grown in a single deposition run creating high quality structures which can be analyzed in situ further reducing 
extrinsic effects due to contamination from the atmosphere which can have a profound effect on the properties of nanoscale structures.

The major characterization technique used in this thesis is Piezoresponse Force Microscopy (PFM). Studying nanoscale ferroelectrics requires a characterization technique that can probe the polarization and ferroelectric properties with a resolution smaller than the length scale of the change in boundary conditions. Being a scanning probe technique the PFM tip has a radius of $\sim 30 \mathrm{~nm}$. However, the electric field produced by the biased tip is smaller than the radius allowing a resolution of less than $30 \mathrm{~nm}$. In Chapter 3 the resolution of the PFM in $\mathrm{UHV}$ and in atmosphere is quantitatively determined using $180^{\circ}$ domain walls, implementing the 25-75 rule and a Boltzmann fit. The resolution of PFM in UHV was $\sim 10 \mathrm{~nm}$ and was found to be a factor of 1.6 better than imaging in atmosphere. Preforming PFM in UHV directly after sample growth had an added benefit of preventing any contamination of the ferroelectric further ruling out extrinsic effects to any observed size effects.

In Chapter 5 ferroelectric $\mathrm{PbTiO}_{3}$ and $\mathrm{BiFeO}_{3}$ thin films of $25 \mathrm{~nm}$ were grown on self-assembled $\mathrm{SrRuO}_{3}$ nanowires which are $5 \mathrm{~nm}$ high, with a periodicity of $400 \mathrm{~nm}$ and run across the substrate surface allowing the wires to be electrically contacted at the sample edge. The conducting $\mathrm{SrRuO}_{3}$ wires are grown on $\mathrm{DyScO}_{3}$ substrates which are insulating thereby changing the electrical boundary conditions for regions of the film on and off the nanowires. In addition, the high aspect ratio of the nanowires means that strain around the corners of the nanowires also changes the mechanical boundary condition for different regions of the film. Of the two ferroelectric materials the $\mathrm{PbTiO}_{3}$ had a strong preference for its domains to align with the underlying nanowires while the $\mathrm{BiFeO}_{3}$ did not. In the $\mathrm{PbTiO}_{3}$ the $180^{\circ}$ domains preferred to align perpendicular to the nanowires and the domains started and stopped at the nanowire edges. The $90^{\circ}$ domains lined up along the nanowire edges and analysis by TEM found that the majority of the $90^{\circ}$ domains originated at the corners of the nanowires. The difference in the domain alignment with the nanowires in the $\mathrm{PbTiO}_{3}$ and $\mathrm{BiFeO}_{3}$ sample corresponded difference in how the film covered the $3 \mathrm{D}$ nanowires. The $\mathrm{BiFeO}_{3}$ was atomically smooth showing no evidence of the nanowire pattern in the surface morphology while the nanowires were clearly visible when covered by the $\mathrm{PbTiO}_{3}$. Studying the growth of the two materials on $\mathrm{SrRuO}_{3}$ nanoislands revealed two different growth modes. The $\mathrm{BiFeO}_{3}$ film would first fill in the space between the nanoislands while the $\mathrm{PbTiO}_{3}$ film grew evenly on or off the $\mathrm{SrRuO}_{3}$. The growth mode coupled with the $\mathrm{T}_{c}$ of the $\mathrm{PbTiO}_{3}$ being higher than the growth temperature explained why domain alignment occurred for this material. The conformal coating of the film over the $\mathrm{SrRuO}_{3}$ nanowires creates a large strain which is relaxed by the formation of $90^{\circ}$ domains. For the $\mathrm{BiFeO}_{3}$ such strain does not occur as the material grows in an essentially $2 \mathrm{D}$ manner.

In Chapter 6 the $\mathrm{SrRuO}_{3}$ nanowires are used as local electrodes to probe the domain wall conductivity. Understanding the nature of domain wall conductivity has proved difficult 
due to the similar response of the current vs bias for several different conduction mechanisms even when temperature dependence is taken into account. Studying how the domain wall conductivity scales with distance provides an easy way to differentiate between bulk or interface limited conduction mechanisms. To date this has been done only by changing the film thickness which is a time consuming process. As a result, thickness dependence data in literature consists of only a few data points making trends difficult to identify. In this thesis using the $\mathrm{SrRuO}_{3}$ nanowires as bottom electrodes and a conducting AFM tip as a top electrode the current along a domain wall running between two nanowires is measured continuously along the domain wall. The current along the domain wall is found to decrease to zero as the domain wall runs off the underlying nanowire. This strongly points to a bulk limited conduction mechanism, either spaced charged limited or Poole-Frenkel hopping. However, the decrease in conductivity was not consistent and is found to decrease exponentially with an exponent of -1.5 or in a series of discreet jumps. Neither of these behaviors are expected from the two bulk limited conduction mechanisms.

In the final chapter, $\mathrm{Ca}_{2} \mathrm{Nb}_{3} \mathrm{O}_{10}$ nanosheets are used as a seed layer for the investigation of size effects in $\mathrm{PbTiO}_{3}$ thin films. The nanosheets are one unit cell thick and range in size from $100 \mathrm{~nm}$ to $1.5 \mu \mathrm{m}$ allowing a wide range of lateral sizes to be studied in a single sample. On the nanosheets epitaxial films are grown while polycrystalline growth occurs off the nanosheets making it possible to identify the original size and shape of the nanosheets after the deposition of the $\mathrm{PbTiO}_{3}$ film. On the nanosheets only $180^{\circ}$ domains are observed and are mostly small circular domains with a size of $\sim 30 \mathrm{~nm}$. Some long straight domains are observed along the edges of the nanowires. Unlike what has been reported in many size effect studies no increase in the piezoresponse was observed in any region of the $\mathrm{PbTiO}_{3}$ on the nanosheets. The response amplitude is uniform within each $\mathrm{PbTiO}_{3}$ covered nanosheets, at the edges between two $\mathrm{PbTiO}_{3}$ covered nanosheets and on the border between a densely covered nanosheet region and $\mathrm{PbTiO}_{3}$ grown on silicon region. The enhancement of the piezoresponse observed in literature is attributed to a relaxation in the strain state as the material becomes unclamped during the patterning process. However, since the $\mathrm{PbTiO}_{3}$ on the nanosheets is not post patterned such unclamping does not take place and no increase of the piezoresponse is observed. 


\section{Samenvatting}

Ferroelektrische materialen vormen een belangrijke materiaalklasse vanwege hun grote scala aan interessante eigenschappen. Toepassingen in bijvoorbeeld condensatoren (vanwege de hoge dielektrische constante), pyroelektrische infrarood detectoren, piëzoelektrische actuatoren en sensoren, Micro Electro Mechanische Systemen (MEMS) en zelfs het gebruik als permanent geheugen in computers geven de veelzijdigheid van ferroelektrische materialen aan en ook de inzetbaarheid in verschillende technologische disciplines. Integratie van ferroelektrische elementen in elektronica is de drijfveer tot miniaturisatie van ferroelektrische componenten om de vraag naar draagbare elektronica met toenemende functionaliteit bij te kunnen houden. Het is echter bekend dat de aantrekkelijke eigenschappen van ferroelektrische materialen degraderen wanneer de materialen in één of meerdere dimensies de nanoschaal grens benaderen. Daarom is het van belang om de eigenschappen van ferroelektrische materialen te begrijpen voor objecten op deze kleine schaal. Zowel het begrijpen als het proberen te behouden van de ferroelektrische eigenschappen wanneer deze begrensd worden op deze schaal is een actief onderzoeksgebied.

Veel studies naar schaaleffecten in ferroelektrische materialen gebruiken "topdown" technieken om ferroelektrische objecten te creëren uit een ferroelektrische film. In veel gevallen wordt "Focused Ion Beam" en "Electron Beam Lithography" gebruikt om tot ferroelektrische objecten te komen met afmetingen kleiner dan 100nm. De gebruikte "topdown" technieken introduceren echter schade an het ferroelektrische materiaal, zoals ionen implantatie en hersputtering. Vanwege de gevoelige relatie tussen de kristal structuur en de eigenschappen van een ferroelektrisch materiaal kan dergelijke schade leiden tot extrinsieke effecten, wat vervolgens de werkelijke invloed van de schaaleffecten op de ferroelektrische eigenschappen kan maskeren. Dergelijke nadelen kunnen voorkomen worden door het gebruikt van "bottom-up" benaderingen omdat hierbij gebruik gemaakt wordt van natuurlijk optredende zelfassemblage processen om tot nanoschaal objecten te komen. Controle over de vorm, grootte en positie van de resulterende nanoschaal objecten blijft een uitdaging.

In dit proefschrift worden de schaaleffecten van ferroelektrische materialen onderzocht op een nieuwe manier door de grenzen van de ferroelektrische objecten te moduleren. Het aanpassen van het ferroelektrische materiaal tot nanoschaal objecten verandert de grenscondities en resulteert in schaaleffecten. Een alternatieve manier om deze schaaleffecten op de nanoschaal te bestuderen is daarom de veranderingen van een ferroelektrische film te onderzoeken wanneer de grenscondities worden aangepast. Dit kan 
gedaan worden door manipulatie op de nanoschaal toe te passen op het onderste grensvlak van de dunne film. Deze aanpak heeft als voordeel dat nabewerkingen van het ferroelektrische materiaal, wat leidt tot extrinsieke effected, niet nodig is. Een ander voordeel van deze aanpak is dat door gebruik te maken van zelfassemblage technieken om het onderste grensvlak te manipuleren, het volledige sample in een enkele depositie stap gegroeid kan worden. Hiermee kunnen dergelijke structuren van hoge kwaliteit gemaakt worden en kunnen de monsters insitu gekarakteriseerd worden wat verdere extrinsieke effecten die een significant effect op de eigenschappen op de nanoschaal kunnen hebben, zoals oppervlaktevervuiling ten gevolge van blootstelling aan lucht, uit kan sluiten.

De belangrijkste karakterisatie techniek die gebruikt is in dit proefschrift is "Piezoresponse Force Microscopy" (PFM). Om ferroelektrische materialen op de nanoschaal te bestuderen is een techniek nodig die de polarisatie en ferroelektrische eigenschappen kan meten met een resolutie die kleiner is dan de lengteschaal waarop de materialen zijn gemanipuleerd. Ondanks dat de PFM tip een radius heeft van ongeveer $30 \mathrm{~nm}$ is het resulterende elektrische veld ten gevolge van het aanbrengen van een spanning op de tip kleiner dan de tip radius, waardoor een kleinere resolutie dan de radius van de tip mogelijk is. In hoofdstuk 3 wordt de resolutie van PFM in zowel UHV als in lucht kwantitatief bepaald door gebruik te maken van $180^{\circ}$ domein grenzen en de 25-75 regel en een Boltzmann benadering. De resolutie van PFM metingen in UHV is ongeveer $10 \mathrm{~nm}$ en is een factor 1.6 beter dan wanneer PFM metingen in lucht worden uitgevoerd. Wanneer PFM in UHV wordt uitgevoerd direct na het groeien van het sample heeft ook als voordeel dat vervuiling van het ferroelektrische materiaal voorkomen wordt. Hierdoor kunnen de daadwerkelijke schaaleffecten gemeten worden in plaats van extrinsieke effecten.

In hoofdstuk 5 zijn ferroelektrische $\mathrm{PbTiO}_{3}$ en $\mathrm{BiFeO}_{3}$ dunne films van $25 \mathrm{~nm}$ gegroeid op zelf geassembleerde $\mathrm{SrRuO}_{3}$ nanodraden, welke $5 \mathrm{~nm}$ hoog zijn en een periodiciteit hebben van $400 \mathrm{~nm}$. Deze nanodraden lopen over het volledige substraatoppervlak waardoor elektrisch contact met deze draden te maken aan de zijkant van het sample. De geleidende $\mathrm{SrRuO}_{3}$ draden zijn gegroeid op $\mathrm{DyScO}_{3}$ substraten die isolerend zijn, wat resulteert in verschillende elektrische omgevingen voor regio's van de dunne film op en naast de nanodraden. Bovendien is, vanwege de hoge aspect ratio van de nanodraden, de vervorming in de buurt van bochten in de nanodraden ook beïnvloedt, wat ook leidt tot variatie van de mechanische omgevingen van verschillende regio's van de dunne film. Van de twee ferroelektrische materialen heeft $\mathrm{PbTiO}_{3}$ een sterke voorkeur om de domeinen uit te lijnen ten opzichte van de onderliggende nanodraden, in tegenstelling tot $\mathrm{BiFeO}_{3}$ waarvoor deze voorkeur niet is waargenomen. In $\mathrm{PbTiO}_{3}$ hebben $180^{\circ}$ domeinen een voorkeursoriëntatie loodrecht op de lengterichting van de nanodraden en de domeinen beginnen en eindigen bij de randen van de nanodraden. De $90^{\circ}$ domeinen lijnen langs de randen van de nanodraden en uit TEM analyse is gebleken dat de meerderheid van de $90^{\circ}$ domeinen beginnen bij de hoeken van de nanodraden. Het verschil in domein oriëntatie ten opzichte van de nanodraden in $\mathrm{PbTiO}_{3}$ 
en $\mathrm{BiFeO}_{3}$ samples correspondeert met hoe de dunne films de 3D nanodraden bedekken. Voor $\mathrm{BiFeO}_{3}$ samples het oppervlak is atomair glad en de onderliggende nanodraden zijn niet meer zichtbaar, terwijl de onderliggende nanodraden nog duidelijk zichtbaar zijn voor $\mathrm{PbTiO}_{3}$ samples. De verschillen in groeimodus werd duidelijk door de groei van beide materialen op $\mathrm{SrRuO}_{3}$ nano-eilanden te bestuderen. $\mathrm{BiFeO} 3$ films vullen eerst de ruimte tussen de nanoeilanden op, terwijl $\mathrm{PbTiO} 3$ gelijkmatig groeit zowel op als naast de nano-eilanden van $\mathrm{SrRuO}_{3}$. De combinatie van de groeimodus en een $\mathrm{T}_{c}$ die hoger is dan de groeitemperatuur verklaren de voorkeursoriëntatie van de domeinen voor $\mathrm{PbTiO}_{3}$. De gelijkmatige bedekking van de dunne film op de $\mathrm{SrRuO}_{3}$ eilanden creëert een grote vervorming die gerelaxeerd wordt door het vormen van $90^{\circ}$ domeinen. Een dergelijke vervorming treedt niet op bij $\mathrm{BiFeO}_{3}$ omdat deze een $2 \mathrm{D}$ groeimodus heeft.

In hoofdstuk 6 worden de $\mathrm{SrRuO}_{3}$ nanodraden gebruikt als lokale elektrodes om de geleiding van de domein grenzen te meten. Het begrijpen van de geleiding van de domein grenzen is moeilijk vanwege een vergelijkbare stroom-spanning relatie van verschillende geleidingsmechanismes, zelfs als deze temperatuursafhankelijk worden bepaald. Het bestuderen van de geleiding van de domein grenzen als functie van de afstand is een makkelijke manier om het verschil te bepalen tussen bulk en grensvlak gelimiteerde geleidingsmechanismes. Dit is echter alleen gedaan door de filmdikte te variëren wat een tijdrovende aanpak is. Als een gevolg hiervan zijn slechts dataseries met enkele datapunten gepubliceerd, wat het erg moeilijk maakt om trends te onderscheiden. In dit proefschrift worden $\mathrm{SrRuO}_{3}$ nanodraden gebruikt als onderste elektrode en een geleidende AFM tip als bovenste elektrode om de stroom te meten langs een domeingrens die tussen twee nanodraden loopt. Op deze manier kan de stroom als functie van de afstand op een continue manier gemeten worden. Uit deze metingen blijkt dat de stroom langs een domein grens afneemt tot nul wanneer de domein grens van de onderliggende nanodraad afgaat. Dit is een sterke indicatie voor een bulk gelimiteerd geleidingsmechanisme, space charged of Poole-Frenkel hopping. Het afnemen van de geleiding is echter niet consistent en neemt exponentieel af met een exponent van -1.5 of in een set van discrete stappen. Geen van deze observaties worden verwacht voor de eerder genoemde bulk gelimiteerde geleidingsmechanismes.

In het laatste hoofdstuk, worden $\mathrm{Ca}_{2} \mathrm{Nb}_{3} \mathrm{O}_{10}$ nanoplaten gebruikt als nucleatie laag om schaaleffecten in $\mathrm{PbTiO}_{3}$ dunne films te onderzoeken. Deze nanoplaten zijn één eenheidscel dik en hebben afmetingen van $100 \mathrm{~nm}$ tot $1.5 \mu \mathrm{m}$, waardoor een grote range van laterale afmetingen bestudeerd kunnen worden in slechts één sample. Op deze nanoplaten worden epitaxiale films gegroeid, terwijl tussen de nanoplaten polikristallijn materiaal wordt gevormd. Dit maakt het mogelijk om na de depositie van de $\mathrm{PbTiO}_{3}$ dunne film het formaat van de onderliggende nanoplaten te herkennen. Op de nanoplaten worden alleen 180 domeinen aangetroffen en deze zijn voornamelijk cirkelvormig met een afmeting van ongeveer $30 \mathrm{~nm}$. Enkele lange rechte domeinen werden aangetroffen langs de randen van de nanodraden. In tegenstelling tot eerdere publicaties van schaaleffecten wordt hier nergens een toename van 
de piëzo-response angetroffen van de $\mathrm{PbTiO}_{3}$ op de nanoplaten. De amplitude response is uniform binnen alle $\mathrm{PbTiO}_{3}$ bedekte nanoplaten, aan de randen tussen twee $\mathrm{PbTiO} 3$ bedekte nanoplaten en op de grens tussen een dicht bedekte regio met nanoplaten en $\mathrm{PbTiO}_{3}$ gegroeid op silicium. De toename van de piëzo-response die in literatuur is beschreven wordt toegekend aan een relaxatie van de vervorming toestand naarmate het materiaal relaxeert gedurende het bewerkingsproces waarin de nanostructuren worden gemaakt. In de studie die hier gepresenteerd wordt van $\mathrm{PbTiO}_{3}$ op nanoplaten vindt er geen nabewerking plaats om tot nanostructuren te komen en ten gevolge hiervan is er dus ook geen toename van de piëzoresponse waargenomen. 


\section{Acknowledgements}

I guess I am better with microscopes than words and sentences. I will try my best to acknowledge all the people I feel so grateful to, but please note that I am not a great writer. Those few sentences probably are less than you all really deserve.

I would first and foremost like to acknowledge my supervisors Gertjan Koster, Andre ten Elshof, and Guus Rijnders without which this thesis would not have been possible. Thank you for the confidence you showed in me by hiring me to work on the Size Effects in Ferroelectrics project. I am grateful for your continued guidance over the four years of my $\mathrm{PhD}$. You encouraged me to define my $\mathrm{PhD}$ on my own terms allowing me the freedom to explore while still making sure that I did not wonder too far astray. Your comments and feedback on my various experiments, data, presentations, abstracts, and papers, helped me develop further as a researcher. Thank you for the time you devoted, your patience and effective advice during times when I struggled and encouragement when things were looking up.

I would like to express my gratitude to my collaborations with the EMAT group at the University of Antwerp and the Center for Nanophase Material Science at Oakridge National Laboratories for the measurements and encouraging discussions. Specifically I would like to thank Ricardo Egoavil for the doing the TEM measurements on the $\mathrm{PbTiO}_{3}$ on the $\mathrm{SrRuO}_{3}$ nanowires, Sara Bals, and Jo Verbeeckfor the many insights from analysis of the resulting TEM and EELS data. Much of the contents of Chapter 3 were the results of measurements done at the Center for Nanophase Material Science at Oakridge National Laboratories. I want to thank Rama Vasudevan for helping me with the actual measurements and Sergei Kalinin and Petro Maksymovych for sharing their thoughts on the results.

I would like to thank the past and present members of the COMAT team (Bouwe, Rik, Tom, Josee, Jost, Hans, Jeroen, Michelle, David) who provided a great working and learning environment in the lab. All these late evenings when we fixed things that were broken were rewarding because our team effort paid off while learning something new and having some fun at the same time. I will always remember one of the biggest challenges and truly unique experience of moving the lab. Moving the lab was huge pain for everyone but we managed and together got to learn the system inside out. Literally!

I would like to acknowledge all the former and current members of the IMS group (Nirupam, Peter B., Gerard, Petra, David, Kenan, Anirban, Pablo, Rik, Michelle, Maarten, Alim, Sjoerd, Kurt, Werner, Tom, Huiyu, Rogier, Wouter, Bouwe, Xin, Ruud, Josee, Jeroen, Anthony, Tomasz, Peter V., Nicolas, Han, Michiel, Oktay, Minh, Ben, Evert, Debakanta, Mark, 
Matthijn) for providing a great working atmosphere throughout the group both from a scientific standpoint was well as a social one. From discussions and feedback during our weekly meetings to trips such as the ski weekend in Germany and the study tour in Oxford the IMS group members certainly made my $\mathrm{PhD}$ experience a memorable one. I am also thankful to my office mates (Bouwe, Nirupam, Josee) for friendly atmosphere and countless cups of coffees we shared along with productive or just fun discussions.

I am grateful to all members of the IMS football team (Peter V., Smithers, Rik, David, Hans, Gerard, Jasper, Ruud, Tim, Mark, Matthijn, Menno, Ben, Kurt). It was great to get out of the lab once a week and have some fun competing against the other research groups and it was always lots of fun, not only when we could celebrate a win. Also, I was always looking forward to the tournament at the end of season with our traditional BBQ afterwards. Thanks for this great experience.

I want to thank my Paranymphs Rik Groenen and Nirupam Banerjee for all their help with before during and after the defense. Also Peter B. for translating my summary into Dutch and Kurt for helping to do it.

Thank you to Marion, José, Dominic, Henk for all they did to make my life and work much easier during my time in Enschede. From filling out forms and official documents to ordering materials to technical help in the lab I would not have had a successful $\mathrm{PhD}$ without your efforts.

Outside of the University I want to express my gratitude to my team members from EFC PW teams 1 and 6 (especially Peter V. for bringing me into the club) as well as the managers of the club. Getting to know another football culture was a great experience and I really enjoyed the atmosphere around EFC PW. From drinking beers while watching the Champions League after trainings to PW1 team outings in Dorf Munsterland to winning the league with PW1 in my final season (although I was very disappointed not to be there for the last $1 / 2$ of the season) it was a fantastic experience. It also helped me to keep sanity and positive perspective during all ups and downs throughout the $\mathrm{PhD}$.

A big thank you to my girlfriend Vilija. Your support during the tough times in my $\mathrm{PhD}$ helped me make it over the finish line. I would certainly not have been able to make such a great thesis book without your help editing my manuscript, designing the cover and invitation.

I am appreciative of my parents and siblings who were always there for me in many ways despite the distance. Finally, I am especially grateful to my parents for providing opportunities while I was growing up to explore and learn. It allowed me to find the two most important things which I am passionate about football and science.

Brian F. Smith

Stuttgart, 2014 